\title{
On the origin of the $\mathrm{O}$ and $\mathrm{B}$-type stars with high velocities
}

\section{Runaway stars and pulsars ejected from the nearby young stellar groups}

\author{
R. Hoogerwerf, J. H. J. de Bruijne, and P. T. de Zeeuw
}

Sterrewacht Leiden, Postbus 9513, 2300 RA Leiden, The Netherlands

Received 17 August 2000 / Accepted 28 September 2000

\begin{abstract}
We use milli-arcsecond accuracy astrometry (proper motions and parallaxes) from Hipparcos and from radio observations to retrace the orbits of 56 runaway stars and nine compact objects with distances less than $700 \mathrm{pc}$, to identify the parent stellar group. It is possible to deduce the specific formation scenario with near certainty for two cases. (i) We find that the runaway star $\zeta$ Ophiuchi and the pulsar PSR J1932+1059 originated about 1 Myr ago in a supernova explosion in a binary in the Upper Scorpius subgroup of the Sco OB2 association. The pulsar received a kick velocity of $\sim 350 \mathrm{~km} \mathrm{~s}^{-1}$ in this event, which dissociated the binary, and gave $\zeta$ Oph its large space velocity. (ii) Blaauw \& Morgan and Gies \& Bolton already postulated a common origin for the runaway-pair AE Aur and $\mu$ Col, possibly involving the massive highly-eccentric binary $\iota$ Ori, based on their equal and opposite velocities. We demonstrate that these three objects indeed occupied a very small volume $\sim 2.5 \mathrm{Myr}$ ago, and show that they were ejected from the nascent Trapezium cluster. We identify the parent group for two more pulsars: both likely originate in the $\sim 50$ Myr old association Per OB3, which contains the open cluster $\alpha$ Persei. At least 21 of the 56 runaway stars in our sample can be linked to the nearby associations and young open clusters. These include the classical runaways 53 Arietis (Ori OB1), $\xi$ Persei (Per OB2), and $\lambda$ Cephei (Cep OB3), and fifteen new identifications, amongst which a pair of stars running away in opposite directions from the region containing the $\lambda$ Ori cluster. Other currently nearby runaways and pulsars originated beyond $700 \mathrm{pc}$, where our knowledge of the parent groups is very incomplete.
\end{abstract}

Key words. Astrometry - stars: early-type - stars: kinematics - pulsars: general - supernova: general

\section{Introduction}

About $10-30 \%$ of the $\mathrm{O}$ stars and $5-10 \%$ of the B stars (Gies 1987; Stone 1991) have large peculiar velocities (up to $200 \mathrm{~km} \mathrm{~s}^{-1}$ ), and are often found in isolated locations; these are the so-called "runaway stars" (Blaauw 1961, hereafter Paper I). The velocity dispersion of the population of runaway stars, $\sigma_{\mathrm{v}} \sim 30 \mathrm{~km} \mathrm{~s}^{-1}$ (e.g., Stone 1991), is much larger than that of the "normal" early-type stars, $\sigma_{\mathrm{v}} \sim 10 \mathrm{~km} \mathrm{~s}^{-1}$. Besides their peculiar kinematics, runaway stars are also distinguished from the normal earlytype stars by an almost complete absence of multiplicity (cf. the binary fraction of normal early-type stars is $>50 \%$ [e.g., Mason et al. 1998]). Furthermore, over $50 \%$ of the (massive) runaways have large rotational velocities and enhanced surface helium abundances (Blaauw 1993).

Several mechanisms have been suggested for the origin of runaway stars (Zwicky 1957; Paper I; Poveda et al. 1967; Carrasco et al. 1980; Gies \& Bolton 1986), two of which are still viable: the binary-supernova scenario (Paper I)

Send offprint requests to: P. T. de Zeeuw,

e-mail: tim@strw.leidenuniv.nl and the dynamical ejection scenario (Poveda et al. 1967). We summarize them in turn.

\section{Binary-supernova scenario (BSS)}

In this scenario a runaway star receives its velocity when the primary component of a massive binary system explodes as a supernova. When the supernova shell passes the secondary the gravitational attraction of the primary reduces considerably, and the secondary starts to move through space with a velocity comparable to its original orbital velocity $\left(30-150 \mathrm{~km} \mathrm{~s}^{-1}\right)$. What remains of the primary after the explosion is a compact object, either a neutron star or a black hole. Depending on the details of the preceding binary evolution, the eccentricity of the orbit, and the kick velocity $\boldsymbol{v}_{\text {kick }}$ due to the asymmetry of the supernova explosion (e.g., Burrows et al. 1995), the compact object may or may not remain bound to the runaway star (e.g., Hills 1983). In most cases less than half of the total mass of the binary is expelled in the explosion. As this is insufficient to break up the binary (Paper I), most BSS runaways are expected to remain double. Examples of 
such systems are provided by the high-mass-X-ray binaries (e.g., van den Heuvel et al. 2000). Their typical velocities of $\sim 50 \mathrm{~km} \mathrm{~s}^{-1}$ (Kaper et al. 1997; Chevalier \& Ilovaisky 1998) are the natural result of the recoil velocity acquired when the supernova shell leaves the binary system.

Several searches failed to find compact companions of classical runaway stars (Gies \& Bolton 1986; Philp et al. 1996; Sayer et al. 1996), suggesting that for these systems $\boldsymbol{v}_{\text {kick }}$ of the neutron star was large enough (several $100 \mathrm{~km} \mathrm{~s}^{-1}$ ) to unbind the binary (e.g., Frail \& Kulkarni 1991; Cordes et al. 1993; Lai 1999). The typical magnitude of this "threshold" kick velocity is uncertain (e.g., Hills 1983; Lorimer et al. 1997; Hansen \& Phinney 1997; Hartman 1997).

Single BSS runaways must originate in close binaries because these systems have the largest orbital velocities, and therefore they have experienced close binary evolution before being ejected as a runaway. This leads to the following observable characteristics:

1: BSS runaways are expected to have increased helium abundance $^{1}$ and large rotational velocity: when the primary fills its Roche lobe, mass and angular momentum is transferred to the star that will become the runaway. The mass transfer stops when the "primary" has become a helium star (i.e., only the helium core remains). This process enriches the runaway with helium, and spins it up (e.g., Packet 1981; van den Heuvel 1985; Blaauw 1993);

2: A BSS runaway can become a blue straggler, because it is rejuvenated during the mass-transfer period through the fresh fuel it receives from the primary;

3: The kinematic age of a BSS runaway star (defined as the time since the runaway left its parent group) should be smaller than the age of the parent group. The primary of the original binary system first evolves for several Myr before it explodes and the runaway is ejected.

\section{Dynamical ejection scenario (DES)}

In this scenario runaway stars are formed through gravitational interactions between stars in dense, compact clusters. Although binary-single star encounters produce runaways (e.g., Hut \& Bahcall 1983), the most efficient interaction is the encounter of two hard binary systems (Hoffer 1983). Detailed simulations show that these collisions produce runaways with velocities up to $200 \mathrm{~km} \mathrm{~s}^{-1}$ (Mikkola 1983a, 1983b; Leonard \& Duncan 1988, 1990; Leonard 1991). The outcome of a binary-binary collision can be (i) two binaries, (ii) one single star and a hierarchical triple system, (iii) two single stars and one binary, and (iv) four single stars (Leonard 1989). In most cases the collision will result in the ejection of two single stars and one hard binary with an eccentric orbit

\footnotetext{
1 The abundances of other elements are increased as well, in particular that of nitrogen which can be enhanced by a factor 4-5 (Van Rensbergen priv. comm.).
}

(Hoffer 1983; Mikkola 1983a). Since the resulting binary is the most massive end product of the collision it is unlikely to gain a lot of speed; it might even remain within the parent cluster. This process naturally leads to a low (0-33\%) runaway binary fraction which is in qualitative agreement with the observations (e.g, Gies \& Bolton 1986). For the DES to be efficient the initial binary fraction in clusters needs to be large. Recent observations show that the binary fraction for massive stars in young clusters is $>50 \%$ (Abt 1983; Kroupa et al. 1999; Preibisch et al. 1999 [approaching 100\%]).

\section{DES runaways have the following characteristics:}

1: DES runaways are formed most efficiently in a highdensity environment, e.g., in young open clusters. They may also originate in OB associations. These birth sites of massive stars are unbound stellar groups and therefore expand (e.g., Blaauw 1952a, 1978; Elmegreen 1983; Kroupa 2000a), so DES runaways must have been ejected very soon after their formation. The kinematic age and the age of the parent association are thus nearly equal;

2: DES runaways are not expected to show signs of binary evolution such as large rotational velocities and increased helium abundance. However, Leonard (1995) suggested that some binary-binary encounters produce runaways consisting of two stars that merged during the interaction. These would have enhanced helium abundances and large rotational velocities (Benz \& Hills 1987, but see Lombardi et al. 1995);

3: DES runaways are expected to be mostly single stars.

Which of the two formation processes is responsible for runaway stars has been debated vigorously. Both mechanisms create stars with large peculiar velocities which enable them to travel far from their parent group: a velocity of $100 \mathrm{~km} \mathrm{~s}^{-1}$ corresponds to $\sim 100 \mathrm{pc}$ in only $1 \mathrm{Myr}$. The relative importance of the two scenarios can be established by (i) studying the statistical properties of the ensemble of runaway stars, or by (ii) investigating individual runaways in detail. The former approach is based on differences in the general runaway characteristics predicted by each scenario. This requires a large, complete database of runaway stars, which is, to date, unavailable (e.g., Moffat et al. 1998). Here we therefore follow the latter, individual approach by retracing the orbits of runaway stars back in time. The objects encountered by a runaway along its path (e.g., an open cluster, an association, other runaways, or a neutron star), and the times at which these encounters occurred, provide information about its formation. Evidence for the BSS as the formation mechanism for single runaway stars is to find a runaway and a neutron star (pulsar) which occupied the same region of space at the same time in the past. Evidence for the DES is to find a common site of origin for the individual components of the encounter, e.g., a pair of runaways and a binary, in a dense star cluster. 
Table 1. The nearby runaway stars and pulsars with accurate astrometry. HIP indicates the number of the runaway star in the Hipparcos Catalogue, $v_{\text {space }}$ indicates the space motion of the runaway star relative to Galactic rotation (in $\mathrm{km} \mathrm{s}^{-1}$ ), and PSR indicates the pulsar identifications

\begin{tabular}{rrrrrrrrrrrrr}
\hline \hline \multicolumn{1}{c}{ HIP } & $v_{\text {space }}$ & HIP & $v_{\text {space }}$ & \multicolumn{1}{c}{ HIP } & $v_{\text {space }}$ & HIP & $v_{\text {space }}$ & HIP & $v_{\text {space }}$ & HIP & $v_{\text {space }}$ & PSR \\
\hline 3478 & 80.4 & 28756 & 196.6 & 43158 & 57.2 & 61602 & 30.2 & 91599 & 44.7 & 101350 & 36.4 & J0826+2637 \\
3881 & 32.1 & 29678 & 63.0 & 45563 & 125.9 & 62322 & 43.9 & 92609 & 31.0 & 102274 & 46.1 & $\mathrm{~J} 0835-4510$ \\
9549 & 107.9 & 30143 & 55.5 & 46928 & 45.3 & 66524 & 112.7 & 94899 & 162.8 & 103206 & 32.3 & $\mathrm{~J} 0953+0755$ \\
10849 & 50.0 & 35951 & 34.9 & 46950 & 32.1 & 69491 & 77.2 & 94934 & 94.0 & 105811 & 38.3 & $\mathrm{~J} 1115+5030$ \\
14514 & 39.4 & 36246 & 32.1 & 48715 & 34.5 & 70574 & 205.3 & 95818 & 34.7 & 106620 & 45.2 & $\mathrm{~J} 1136+1551$ \\
18614 & 64.9 & 38455 & 41.4 & 48943 & 35.2 & 76013 & 69.0 & 96115 & 165.6 & 109556 & 74.0 & $\mathrm{~J} 1239+2453$ \\
20330 & 34.7 & 38518 & 31.1 & 49934 & 31.2 & 81377 & 23.5 & 97774 & 35.0 & & $\mathrm{~J} 1456-6843$ \\
22061 & 86.5 & 39429 & 62.4 & 52161 & 34.8 & 82171 & 62.9 & 97845 & 70.3 & & $\mathrm{~J} 1932+1059$ \\
24575 & 113.3 & 40341 & 61.7 & 57669 & 31.1 & 82868 & 30.3 & 99435 & 39.4 & & $\mathrm{Geminga}$ \\
27204 & 107.8 & 42038 & 31.3 & 59607 & 78.8 & 86768 & 30.1 & 99580 & 55.6 & & \\
\hline \hline
\end{tabular}

The individual approach requires highly accurate positions $(\alpha, \delta, \pi)$ and velocities $\left(\mu_{\alpha *}, \mu_{\delta}, v_{\text {rad }}\right)$. Here $\alpha$ denotes right ascension, $\delta$ declination, $\pi$ parallax, $\mu_{\alpha *}=$ $\mu_{\alpha} \cos \delta$ proper motion in right ascension, $\mu_{\delta}$ proper motion in declination, and $v_{\text {rad }}$ the radial velocity. The milliarcsecond (mas) accuracy of Hipparcos astrometry (ESA 1997) allows specific investigations of the runaway stars within $\sim 700$ pc. Positions and proper motions of similar accuracy are now available as well for some pulsars through timing measurements and VLBI observations (e.g., Taylor et al. 1993; Campbell 1995). The Hipparcos data also significantly improved and extended the membership lists of the nearby OB associations (de Zeeuw et al. 1999), and of some nearby young open clusters. The resulting improved distances and space velocities of these stellar aggregates make it possible to connect the runaways and pulsars to their parent group, and, in some cases, to identify the specific formation scenario (Hoogerwerf et al. 2000; de Zeeuw et al. 2000). Pre-Hipparcos data (e.g., Blaauw \& Morgan 1954; Paper I; Blaauw 1993; van Rensbergen et al. 1996) allowed identification of the parent groups for some runaways, but generally lacked the accuracy to study the orbits of the runaways in detail (but see Blaauw \& Morgan 1954; Gies \& Bolton 1986).

We define a sample of nearby runaways and pulsars with good astrometry in Sect. 2, and then analyse two cases in depth: $\zeta$ Oph and PSR J1932+1059 in Sect. 3 and AE Aur, $\mu \mathrm{Col}$ and $\iota$ Ori in Sect. 4. We apply the method developed in these sections to the entire sample of runaways and pulsars in Sects. 5, 6, and 7. We discuss helium abundances, rotational velocities and the blue straggler nature of runaways in Sects. 8 and 9, and summarize our conclusions in Sect. 10.

\section{Nearby runaway stars and pulsars}

The parent group is known for about a dozen "classical" runaway stars (Paper I; Blaauw 1993). The Hipparcos Catalogue contains these stars, as well as many additional $\mathrm{O}$ and $\mathrm{B}$ stars which were known in 1982 to have large radial velocities, including 153 of the 162 runaway candidates in Hipparcos Proposal $141^{2}$ (de Zeeuw et al. 1999). Many of these objects are located beyond $\sim 700 \mathrm{pc}$, where the Hipparcos parallax measurement is of modest quality. For this reason we restricted ourselves to a sample of nearby runaway stars, and added to this the (few) nearby pulsars with measured proper motions.

\subsection{Selection of the sample}

We started with all 1118 O to B5 stars in the Hipparcos Catalogue which have radial velocities listed in the Hipparcos Input Catalogue (Turon et al. 1992). Next we only considered those stars which have significant parallaxes $\left(\pi-2 \sigma_{\pi}>0\right.$ mas $)$ and proper motions $\left(\sigma_{\mu} / \mu \leq 0.1\right)$, and space velocities larger than $30 \mathrm{~km} \mathrm{~s}^{-1}$ with respect to the standard of rest of the runaway. For the last requirement we corrected the runaway velocity for Solar motion and Galactic rotation (Dehnen \& Binney 1997). The somewhat arbitrary choice of the velocity limit of $30 \mathrm{~km} \mathrm{~s}^{-1}$ minimizes the contamination of the sample by normal $\mathrm{O}$ and B stars (Sect. 1). These criteria yield 54 runaway candidates (five of which are classical runaways) ${ }^{3}$. This new sample does not contain the nearby runaways $\zeta$ Oph and $\xi$ Per. The former is not selected because its space velocity is smaller than $30 \mathrm{~km} \mathrm{~s}^{-1}$ (although its velocity relative to its parent group Sco OB2 is larger, cf. Sect. 3) and the latter is not selected because $\sigma_{\mu} / \mu>0.1$. However, since the runaway nature of these two stars is well established (e.g., Paper I) we included them in our sample, bringing the total to 56. The Hipparcos numbers and space velocities of these 56 stars are listed in Table 1. Panel $a$ of Fig. 1 shows the histogram of the derived space velocities.

We selected a sample of nearby pulsars from the Taylor, Manchester \& Lyne (1993) catalogue, as updated

\footnotetext{
${ }^{2}$ From a list of $\mathrm{O}$ and $\mathrm{B}$ stars with $v_{\mathrm{rad}}>30 \mathrm{~km} \mathrm{~s}^{-1}$ provided by the late Jan van Paradijs.

3 The sample criteria exclude the known runaways, e.g., $\alpha$ Cam, 68 Cyg (Blaauw 1993), HIP 35707 (Comerón et al. 1998), and HIP 60350 (Maitzen et al. 1998) which all have distances larger than $1 \mathrm{kpc}$.
} 

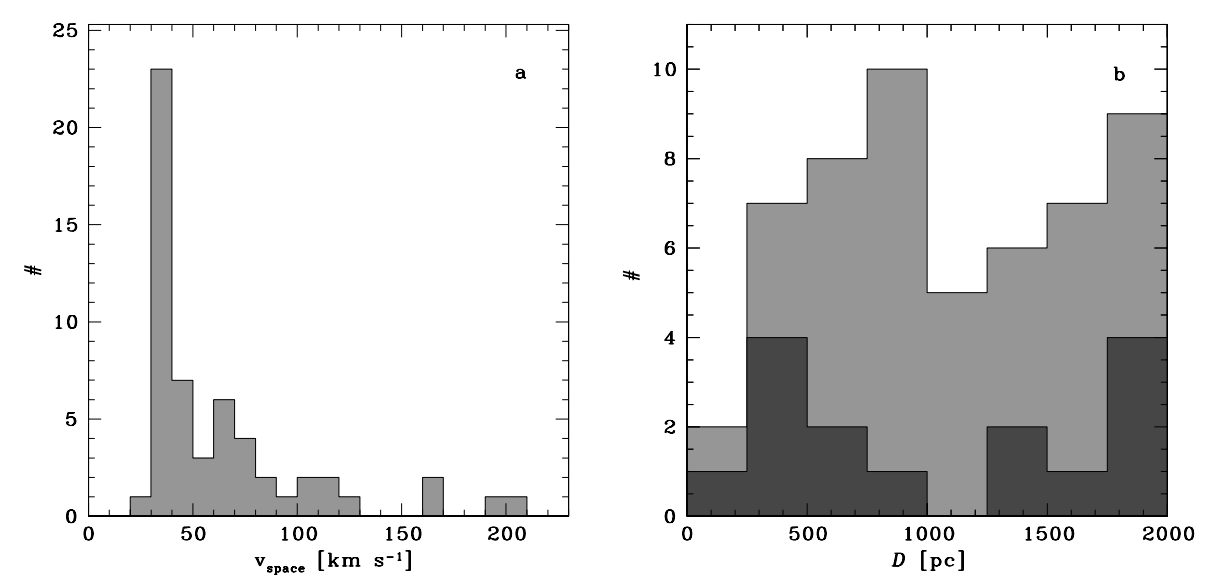

Fig. 1. a) Histogram of the space motions of the sample of runaway stars defined in Sect. 2.1. b) Distribution of pulsars from the Taylor et al. (1993) catalogue with measured proper motions. The light grey histogram shows all pulsars within 2 kpc and the dark grey histogram shows the pulsar with accurate proper motions $\left(\sigma_{\mu} / \mu<0.1\right)$. The latter, for $D<1 \mathrm{kpc}$, is the pulsar sample defined in Sect. 2.1

on http://pulsar.princeton.edu/. It contains 94 pulsars with known proper motions and distances. Only seven of these meet our distance $(D \lesssim 1 \mathrm{kpc})$ and proper motion $\left(\sigma_{\mu} / \mu<0.1\right)$ constraints (see panel $b$ of Fig. 1). Most pulsar distances are derived from the dispersion measure. These distances are unreliable, especially for nearby objects, since they depend on the local properties of the ISM. For one nearby pulsar, PSR J0953+0755, high precision VLBA measurements became available recently (Brisken et al. 2000). We added this pulsar to our sample. The eight pulsars are listed in Table 1, together with Geminga, a nearby neutron star which is not a pulsar, for which an accurate proper motion is known (Caraveo et al. 1996).

Our sample of nearby runaway stars and compact objects is severely incomplete. The Hipparcos Catalogue is complete to $V=7.3-9 \mathrm{mag}$, with the limit depending on Galactic latitude and spectral type (2163 of the $3622 \mathrm{O}$ to B5 stars have $V>7.3 \mathrm{mag})$. The data available for the $\mathrm{O}$ and $\mathrm{B}$ stars is inhomogeneous and incomplete, e.g., less than a third of the O to B5 stars in the Catalog has a measured radial velocity. We have excluded those with large $v_{\text {rad }}$ but insignificant proper motions, as their retraced orbits are uncertain. The beamed nature of the radio emission from pulsars hides many from observation, and not all of those that do radiate in our direction have been found. Of these, only a few have an accurately measured proper motion and a reliable distance.

\subsection{Nearby $O B$ associations and open clusters}

We adopt the positions and mean space motions of the OB associations within $700 \mathrm{pc}$ of the Sun as derived by de Zeeuw et al. (1999) from Hipparcos measurements. For the open clusters we compiled a list from the WEBDA catalogue (http: //obswww . unige.ch/webda/), and consider only those which are young $(\tau<50$ Myr) and with distances less than $700 \mathrm{pc}$ as likely parent groups. The age requirement is comparable to the age of the oldest runaways we consider here (B5V). Typical pulsar ages are less than 50 Myr (e.g., Blaauw \& Ramachandran 1998). This selection yields nineteen open clusters (see Table 2), of which five are already covered in the study of the nearby associations by de Zeeuw et al. (1999). To obtain the space motion of these clusters we use the WEBDA member stars listed in the Hipparcos Catalogue to obtain reliable astrometry, and those in the Hipparcos Input Catalogue to obtain the radial velocity. In this way we are able to construct a more or less reliable space motion for seven of the fourteen remaining open clusters (those labeled "Y" or "?" in Table 2 which summarizes the results).

\subsection{Orbits}

Traditionally, the orbits of runaway stars have been traced back in time using straight lines through space. This is sufficiently accurate for identification of the parent group for times up to a few Myr and distances less than a few hundred pc. To make sure we include the effect of the Galactic potential, we use a fourth-order Runge-Kutta numerical integration method, with a fixed time-step of $10000 \mathrm{yr}$, to calculate the orbit. The Galactic potential we use consists of (i) a logarithmic potential for the halo, (ii) a Miyamoto-Nagai potential for the disk, and (iii) a Plummer potential for the bulge of the Galaxy. The potential predicts Oort constants $A=13.5 \mathrm{~km} \mathrm{~s}^{-1} \mathrm{kpc}^{-1}$ and $B=-12.4 \mathrm{~km} \mathrm{~s}^{-1} \mathrm{kpc}^{-1}$ and a circular velocity $v_{\text {circ }}=219.8 \mathrm{~km} \mathrm{~s}^{-1}$ at $R_{0}=8.5 \mathrm{kpc}$. These values agree with those which Feast \& Whitelock (1997) obtained using Hipparcos data: $A=14.82 \pm 0.84 \mathrm{~km} \mathrm{~s}^{-1} \mathrm{kpc}^{-1}, B=$ $-12.37 \pm 0.64 \mathrm{~km} \mathrm{~s}^{-1} \mathrm{kpc}^{-1}, v_{\text {circ }}=231.2 \pm 16.2 \mathrm{~km} \mathrm{~s}^{-1}$ at $R_{0}=8.5 \mathrm{kpc}$. Since the volume covered in the orbit integration is typically a few hundred pc, and the time of the 
Table 2. The nearby $(D<700 \mathrm{pc})$, young $(\tau<50 \mathrm{Myr})$, open clusters listed in the WEBDA Catalogue. The table gives the designation of the open cluster (Name), its position on the sky $(\ell, b)$, the number of member stars contained in the Hipparcos (HIP) and Hipparcos Input Catalogues (HIC), its distance, proper motion, and radial velocity as obtained from the Hipparcos data $\left(D,\left[\mu_{\ell *}, \mu_{b}\right], v_{\mathrm{rad}}\right)$, and whether the cluster is a candidate parent group in this study (Cand.). The candidate status is denoted by "Y" for the clusters with well-determined positions and velocities, by "N" for clusters for which not all information is available (either astrometry or radial velocity), by " $\mathrm{N}^{*}$ " if the astrometry does not show a clear signature of an open cluster (i.e., a clump in the proper-motion vs. proper-motion diagram), or by "?" if the measurements are not very reliable (either because of a small number of member stars or a large spread in the data)

\begin{tabular}{|c|c|c|c|c|c|c|c|c|c|}
\hline Name & $\begin{array}{c}\ell \\
{[\mathrm{deg} .]}\end{array}$ & $\begin{array}{c}b \\
{[\mathrm{deg} \cdot]}\end{array}$ & $\begin{array}{l}\mathrm{HIP} \\
{[\#]}\end{array}$ & $\begin{array}{c}\mathrm{HIC} \\
{[\#]}\end{array}$ & $\begin{array}{c}D \\
{[\mathrm{pc}]}\end{array}$ & $\begin{array}{c}\mu_{\ell *} \\
{\left[\operatorname{mas~yr}^{-1}\right]}\end{array}$ & 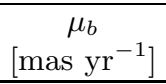 & $\begin{array}{c}v_{\mathrm{rad}} \\
{\left[\mathrm{km} \mathrm{s}^{-1}\right]}\end{array}$ & Cand. \\
\hline Collinder 359 & 29.75 & 12.54 & 10 & - & & & & & $\mathrm{N}^{*}$ \\
\hline IC 4665 & 30.61 & 17.08 & 13 & 5 & $385 \pm 40$ & $-7.2 \pm 0.3$ & $-3.0 \pm 0.3$ & $-13.5 \pm 3.0$ & $\mathrm{Y}$ \\
\hline Stephenson 1 & 66.85 & 15.51 & 0 & - & & & & & $\mathrm{N}$ \\
\hline Roslund 5 & 71.40 & 0.25 & 13 & - & & & & & $\mathrm{N}^{*}$ \\
\hline Stock 7 & 134.68 & 0.04 & 3 & - & & & & & $\mathrm{N}^{*}$ \\
\hline $\begin{array}{l}\alpha \text { Persei } \\
\text { IC } 0348\end{array}$ & \multicolumn{9}{|c|}{$\begin{array}{c}\text { Central part of the Per OB3 association, contained in the list of de Zeeuw et al. } \\
\text { Associated with Per OB2 }\end{array}$} \\
\hline Collinder 69 & 195.05 & -12.00 & 6 & - & $\lambda$ Ori clus & & & & $\mathrm{N}^{*}$ \\
\hline NGC 1976 & \multicolumn{9}{|c|}{ Trapezium cluster: associated with Ori OB1 } \\
\hline NGC 2232 & 214.36 & -47.65 & 10 & 3 & $365 \pm 40$ & $0.7 \pm 0.5$ & $-5.2 \pm 0.5$ & $14.6 \pm 3.0$ & ? \\
\hline Collinder 121 & \multicolumn{9}{|c|}{ Contained in list of nearby associations of de Zeeuw et al. } \\
\hline Collinder 140 & 245.18 & -7.87 & 14 & 4 & $375 \pm 40$ & $-7.4 \pm 0.5$ & $-5.5 \pm 0.5$ & $22.4 \pm 3.0$ & Y \\
\hline Collinder 135 & 248.76 & -11.20 & 19 & 4 & $300 \pm 30$ & $-10.3 \pm 0.5$ & $-6.8 \pm 0.5$ & $16.4 \pm 3.0$ & Y \\
\hline Pismis 5 & 259.39 & 0.86 & 0 & - & & & & & $\mathrm{N}$ \\
\hline Pismis 4 & 262.74 & -2.37 & 4 & 0 & & & & & $\mathrm{~N}$ \\
\hline Trumpler 10 & \multicolumn{9}{|c|}{ Contained in list of nearby associations of de Zeeuw et al. } \\
\hline IC 2391 & 270.36 & -6.88 & 24 & 13 & $150 \pm 30$ & $-33.1 \pm 0.5$ & $-6.0 \pm 0.5$ & $15.0 \pm 3.0$ & Y \\
\hline vdB-Hagen 99 & 286.56 & -0.63 & 7 & 2 & $500 \pm 50$ & $-13.1 \pm 0.5$ & $-6.4 \pm 0.5$ & $12.0 \pm 3.0$ & $?$ \\
\hline IC 2602 & 289.60 & -4.90 & 25 & 8 & $140 \pm 10$ & $-20.4 \pm 0.5$ & $1.2 \pm 0.5$ & $24.1 \pm 3.0$ & Y \\
\hline
\end{tabular}

integration is typically less than $10 \mathrm{Myr}$, perturbations of the orbits caused by small-scale structure in the disk are negligible.

Before integrating the orbit, we correct the observed velocity $\boldsymbol{v}_{*}$ for (i) the Solar motion with respect to the Local Standard of Rest, $\boldsymbol{v}_{\mathrm{lsr}}$ (Dehnen \& Binney 1997), and (ii) the Galactic rotational velocity of the Local Standard of Rest, $\boldsymbol{v}_{\mathrm{gr}}$ (Binney \& Tremaine 1987, p. 14). The stellar velocity $\boldsymbol{v}_{\text {gal }}$ relative to the Galactic reference frame is then given by

$\boldsymbol{v}_{\mathrm{gal}}=\boldsymbol{v}_{*}+\boldsymbol{v}_{\mathrm{lsr}}+\boldsymbol{v}_{\mathrm{gr}}$.

To retrace the orbit, we reverse the velocity and integrate forward in time. We calculate the distance of a star as $1 / \pi$, where $\pi$ is the trigonometric parallax. Since we use the individual parallax, we cannot correct this distance for possible biases (e.g., Smith \& Eichhorn 1996).

\subsection{Identification of parent groups}

We calculate the past orbit of each of the 56 runaway stars listed in Table 1 for 10 Myr. We do this 10000 times for each star, in order to sample the error ellipsoid of the measured parameters, defined by the covariance matrix of the Hipparcos astrometry and the error in the radial velocity measurement. Retracing the orbit of a pulsar is more difficult, because the radial velocity is unknown. We therefore cover a range of radial velocities of $v_{\text {rad }}=0 \pm 500 \mathrm{~km} \mathrm{~s}^{-1}$ in the orbit integrations for the pulsars. Figure 2 shows the positions of the runaways and pulsars on the sky, together with their orbits, retraced back for only $2 \mathrm{Myr}$ so as not to confuse the diagram. Three orbits are shown for each pulsar: for $v_{\text {rad }}=0 \mathrm{~km} \mathrm{~s}^{-1}$ (filled square), $v_{\text {rad }}=200 \mathrm{~km} \mathrm{~s}^{-1}$ (open square), and $v_{\text {rad }}=-200 \mathrm{~km} \mathrm{~s}^{-1}$ (open star).

We also retrace the orbits of the set of nearby OB associations and open clusters defined in Sect. 2.2. These groups have typical linear dimensions of $10-30 \mathrm{pc}$. We consider a group to be a possible site of origin for a runaway or pulsar if the minimum separation between the runaway/pulsar and the group was less than 10 pc at some time in the past $10 \mathrm{Myr}$. With this definition, we find a parent group for 21 of the 56 runaways. These stars are indicated by the filled circles in Fig. 2, and include the seven classical runaways in the sample. We discuss them in detail in Sects. 3-6 below. Six of the nine neutron stars possibly traversed one of the nearby stellar groups; these are PSR J0826+2637, PSR J0835-4510, PSR J1115+5030, PSR J1239+2453, PSR J1932+1059, and Geminga (objects $1,2,4,6,8$, and 9 in Fig. 2). We discuss them in Sects. 3 and 6 , and identify the parent group for four of them. Table 3 summarizes the data for the 22 runaways, four pulsars, and Geminga. The pulsars and runaways for which we cannot identify a parent group are discussed further in Sect. 7. 

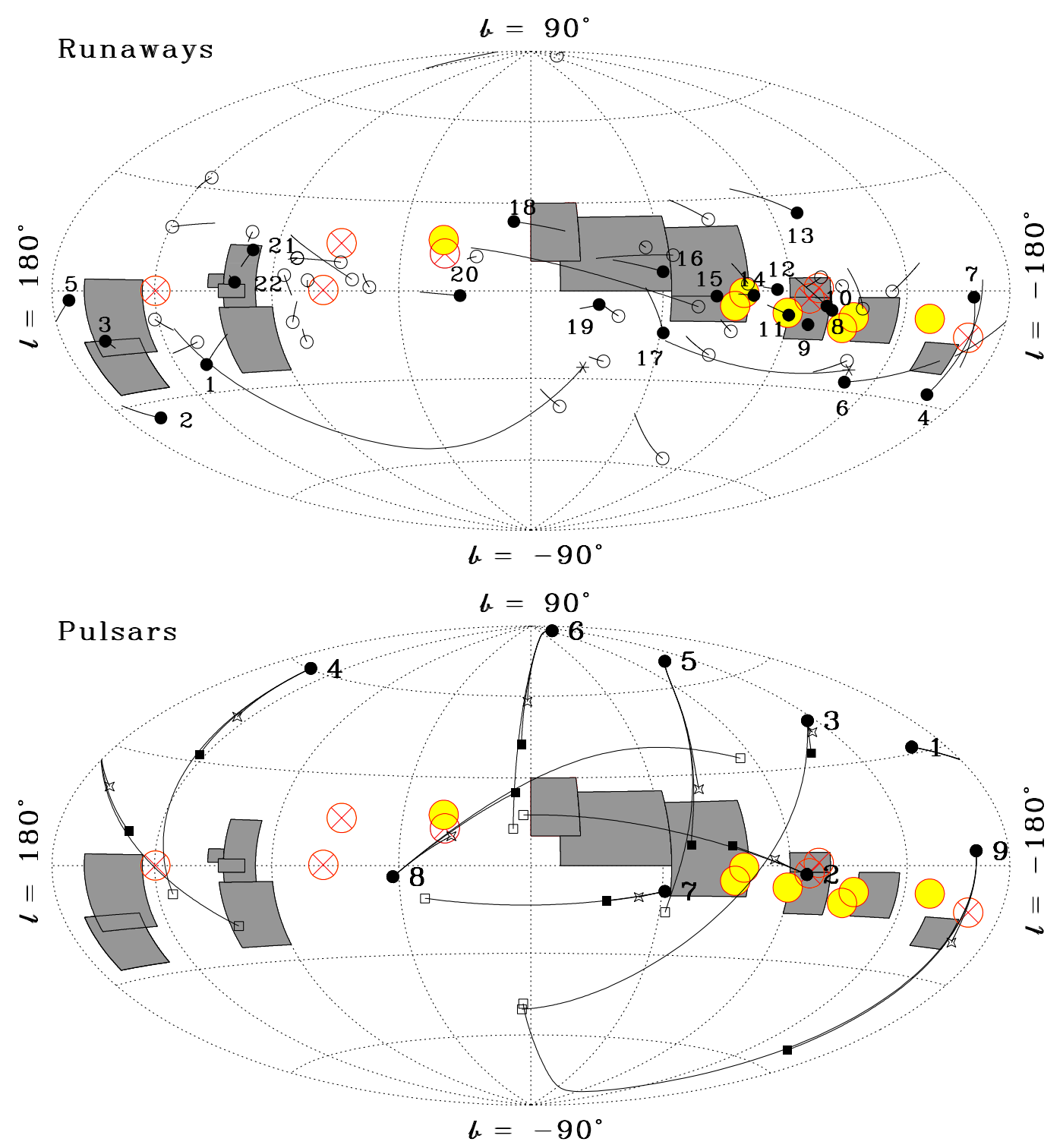

Fig. 2. Top: Sample of runaway stars defined in Sect. 2.1, in Galactic cordinates. The open circles denote the present positions of the runaways, and the arcs show their past orbits, calculated for $2 \mathrm{Myr}$. The filled circles are the runaways for which we can identify the parent association. The numbers refer to the entries in Table 3. The asterisks indicate two additional runaways (72 Col, HIP 94899 [left most of the two asterisks]) discussed in Sect. 7. The grey fields outline the nearby OB associations (de Zeeuw et al. 1999). From left to right and from top to bottom: Per OB3 ( $\alpha$ Persei), Per OB2, Cep OB3, Cep OB2, Cep OB6, Lac OB1, Upper Scorpius, Upper Centaurus Lupus, Lower Centaurus Crux, Tr 10, Vel OB2, Col 121, and Ori OB1. The open clusters are identified by the filled light-grey circles for those with reliable positions and velocities, and by the open, crossed circles for the remaining clusters. The positions and designations of the clusters can be found in Table 2. Bottom: Pulsar sample defined in Sect. 2.1, in Galactic coordinates. The filled circles indicate the present positions of the pulsars. The past orbits of pulsars, calculated for $2 \mathrm{Myr}$, are shown for three different assumed radial velocities: $0 \mathrm{~km} \mathrm{~s}^{-1}$ (filled squares), $200 \mathrm{~km} \mathrm{~s}{ }^{-1}$ (open squares), $-200 \mathrm{~km} \mathrm{~s}^{-1}$ (open stars). The pulsars are labeled 1 through 8; 1: J0826+2637, 2: J0835-4510 (Vela pulsar), 3: J0953+0755, 4: J1115+5030, 5: J1136+1551, 6: J1239+2453, 7: J1456-6843, 8: J1932+1059. Number 9 is the neutron star Geminga. The associations and open clusters typically move comparatively little in $2 \mathrm{Myr}$

\section{A binary supernova in Upper Scorpius}

\section{1. $\zeta$ Oph and PSR J1932+1059}

$\zeta$ Oph is a single O9.5Vnn star, and was first identified as a runaway originating in the Sco OB2 association by Blaauw (1952b). Based on its proper motion, which points away from the association, its radial velocity, and the large space velocity $\left(\sim 30 \mathrm{~km} \mathrm{~s}^{-1}\right)$, Blaauw suggested that $\zeta$ Oph might have formed in the center of the association $\sim 3 \mathrm{Myr}$ ago. Later investigations (e.g., Paper I; Blaauw 1993; van Rensbergen et al. 1996) showed that $\zeta$ Oph either became a runaway $\sim 1$ Myr ago in the Upper Scorpius subgroup of Sco OB2, or 2-3 Myr ago in the Upper Centaurus Lupus subgroup (cf. de Zeeuw et al. 1999).

If $\zeta$ Oph is a BSS runaway, as suggested by its high helium abundance $(\epsilon=0.16$, corresponding to a mass 
Table 3. Data for the nearby runaway stars and pulsars discussed in this paper. The stars which have an * appended to their HIP identifier are the classical runaways (i.e., a parent group was already known before this study, see Paper I). Unless indicated otherwise, the position $(\alpha, \delta)$, proper motion $\left(\mu_{\alpha *}, \mu_{\delta}\right)$, and parallax $(\pi)$ were taken from the Hipparcos Catalogue (ESA 1997), the radial velocity ( $\left.v_{\text {rad }}\right)$ from the Hipparcos Input Catalogue (Turon et al. 1992), the space velocity ( $\left.v_{\text {space }}\right)$ with respect to the standard of rest of the runaway, the rotational velocity $\left(v_{\text {rot }} \sin i\right)$ for the runaways from Penny (1996) and the period $P$ for the pulsars in seconds, the spectral type from Mason et al. (1998) or the Hipparcos Catalogue for the runaways and the characteristic age $(\tau=P /(2 P))$ for the pulsars, and the helium abundance $(\epsilon)$ from Herrero et al (1992), defined as the number of He atoms relative to $\mathrm{H}$. The mass $M_{\mathrm{SK}}$ has been derived from the Schmidt-Kaler (1982) calibration, using interpolation. The mass $M_{\mathrm{BB}}$ is taken from Vanbeveren et al. (1998). The last column (N) indicates the number of the runaway/pulsar in Fig. 2. The proper motion and radial velocity are not corrected for Solar motion and Galactic rotation. The astrometric data $\left(\alpha, \delta, \pi, \mu_{\alpha *}\right.$, and $\left.\mu_{\delta}\right)$ for the pulsars (the last five lines) are taken from the Taylor et al. (1993) catalogue. Abbreviations used: mas $=$ milli-arcsec; $\mu_{\alpha *}=\mu_{\alpha} \cos \delta$

\begin{tabular}{|c|c|c|c|c|c|c|c|c|c|c|c|c|c|c|c|}
\hline HIP & $\mathrm{HD}$ & Name & $\begin{array}{c}\alpha(\mathrm{h} \mathrm{m} \mathrm{s}) \\
{[\mathrm{J} 1991.25]}\end{array}$ & 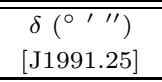 & $\left.\begin{array}{c}\pi \\
{[\mathrm{mas}]}\end{array}\right]$ & $\begin{array}{c}\mu_{\alpha *} \\
{\left[\mathrm{mas} \mathrm{yr}^{-1}\right]}\end{array}$ & $\begin{array}{c}\mu_{\delta} \\
{\left[\operatorname{mas~yr}^{-1}\right]}\end{array}$ & $\begin{array}{c}v_{\mathrm{rad}} \\
{\left[\mathrm{km} \mathrm{s}^{-1}\right]}\end{array}$ & $\begin{array}{c}v_{\text {space }} \\
{\left[\mathrm{km} \mathrm{s}^{-1}\right]}\end{array}$ & $\begin{array}{l}v_{\text {rot } \sin i} \\
{\left[\mathrm{~km} \mathrm{~s}^{-1}\right]}\end{array}$ & $\mathrm{SpT}$ & $\begin{array}{l}M_{\mathrm{SK}} \\
{\left[M_{\odot}\right]} \\
\end{array}$ & $\begin{array}{l}M_{\mathrm{BB}} \\
{\left[M_{\odot}\right]} \\
\end{array}$ & $\begin{array}{c}\epsilon \\
{[\#]}\end{array}$ & $\mathrm{N}$ \\
\hline 3881 & 4727 & $\nu$ And & 04948.83 & +410444.2 & $4.80 \pm 0.75$ & $22.68 \pm 0.53$ & $-18.05 \pm 0.48$ & $-23.9 \pm 1.2$ & 32.1 & $80^{a}$ & $\mathrm{~B} 5 \mathrm{~V}+\mathrm{F} 8 \mathrm{~V}$ & $6.9^{b}$ & & & 1 \\
\hline $14514^{*}$ & 19374 & 53 Ari & 30725.69 & +175247.9 & $4.32 \pm 0.98$ & $-23.54 \pm 0.93$ & $9.30 \pm 0.95$ & $21.2 \pm 1.2^{c}$ & 39.4 & $10^{d}$ & $\mathrm{~B} 1.5 \mathrm{~V}$ & 10.4 & 8.5 & & 2 \\
\hline $18614^{*}$ & 24912 & $\xi$ Per & 35857.90 & +354727.7 & $1.84 \pm 0.70$ & $1.92 \pm 0.74$ & $2.30 \pm 0.62$ & $58.8 \pm 5.0^{e}$ & 64.9 & 204 & O7.5III & 33.8 & 33.5 & 0.18 & 3 \\
\hline $24575^{*}$ & 34078 & AE Aur & $\begin{array}{lll}5 & 16 & 18.15\end{array}$ & +341844.0 & $2.24 \pm 0.74$ & $-4.05 \pm 0.66$ & $43.22 \pm 0.44$ & $57.5 \pm 1.2$ & 113.3 & 25 & $09.5 \mathrm{~V}$ & 15.9 & 21.1 & 0.09 & 5 \\
\hline 26241 & 37043 & $\iota$ Ori & 53525.98 & -055435.6 & $2.46 \pm 0.77$ & $2.27 \pm 0.65$ & $-0.62 \pm 0.47$ & $28.7 \pm 1.1^{f}$ & 8.0 & $71^{g}$ & O9III+B1III ${ }^{h}$ & $37.8^{i}$ & 38.6 & & \\
\hline $27204^{*}$ & 38666 & $\mu \mathrm{Col}$ & 54559.89 & -321823.0 & $2.52 \pm 0.55$ & $3.01 \pm 0.52$ & $-22.62 \pm 0.50$ & $109.0 \pm 2.5$ & 107.8 & 111 & $09.5 \mathrm{~V}$ & 15.9 & 21.1 & & 6 \\
\hline 29678 & 43112 & & 61508.46 & +135103.9 & $2.38 \pm 0.72$ & $24.21 \pm 0.76$ & $10.65 \pm 0.49$ & $36.0 \pm 5.0$ & 63.0 & $<25^{j}$ & B1V & 11.5 & 12.0 & & 7 \\
\hline 38455 & 64503 & & 75238.65 & -385146.2 & $5.09 \pm 0.52$ & $-9.49 \pm 0.43$ & $4.02 \pm 0.42$ & $-31.0 \pm 5.0$ & 41.4 & $212^{k}$ & $\mathrm{~B} 2 \mathrm{~V}$ & 9.4 & 8.0 & & 8 \\
\hline 38518 & 64760 & & 75318.16 & $\begin{array}{lll}-48 & 06 & 10.6\end{array}$ & $1.68 \pm 0.50$ & $-4.90 \pm 0.53$ & $5.89 \pm 0.38$ & $41.0 \pm 5.0$ & 31.1 & $220^{d}$ & B0.5Iab & 25.0 & 35.1 & & 9 \\
\hline 39429 & 66811 & $\zeta$ Pup & 80335.07 & -400011.5 & $2.33 \pm 0.51$ & $-30.82 \pm 0.44$ & $16.77 \pm 0.41$ & $-23.9 \pm 1.2$ & 62.4 & 203 & O4I & & 67.5 & $0.14^{l}$ & 10 \\
\hline 42038 & 73105 & & 83409.60 & $\begin{array}{lll}-53 & 04 & 17.5\end{array}$ & $2.87 \pm 0.47$ & $-12.14 \pm 0.54$ & $10.13 \pm 0.48$ & $37.0 \pm 10.0$ & 31.3 & & B3V & 7.9 & 7.0 & & 11 \\
\hline 46950 & 83058 & & 93408.80 & $\begin{array}{llll}-51 & 15 & 19.0\end{array}$ & $3.50 \pm 0.53$ & $-8.50 \pm 0.49$ & $6.39 \pm 0.48$ & $35.0 \pm 10.0$ & 32.1 & & B1.5IV & $10.4^{m}$ & 9.0 & & 12 \\
\hline 48943 & 86612 & & 95906.32 & -235702.8 & $5.19 \pm 0.77$ & $-23.22 \pm 0.70$ & $5.30 \pm 0.70$ & $39.0 \pm 5.0$ & 35.2 & $230^{d}$ & B5V & 5.8 & & & 13 \\
\hline 49934 & 88661 & & 101146.47 & $\begin{array}{llll}-58 & 03 & 38.0\end{array}$ & $2.52 \pm 0.50$ & $-10.71 \pm 0.49$ & $6.63 \pm 0.45$ & $31.0 \pm 10.0$ & 31.2 & $280^{d}$ & B2IVnpe & $9.4^{m}$ & 8.0 & & 14 \\
\hline 57669 & 102776 & & 114941.09 & $-6347 \quad 18.6$ & $7.10 \pm 0.69$ & $-17.93 \pm 0.95$ & $4.44 \pm 0.63$ & $29.0 \pm 2.5$ & 31.1 & $251^{n}$ & B3V & 7.9 & 7.0 & & 15 \\
\hline 69491 & 124195 & & 141339.84 & -543732.2 & $2.96 \pm 0.63$ & $-18.03 \pm 0.41$ & $-11.15 \pm 0.41$ & $66.0 \pm 10.0$ & 77.2 & & $\mathrm{~B} 5 \mathrm{~V}$ & 5.8 & & & 16 \\
\hline 76013 & 137387 & $\kappa^{1} \mathrm{Aps}$ & 153130.82 & $\begin{array}{lll}-73 & 2322.4\end{array}$ & $3.20 \pm 0.59$ & $0.38 \pm 0.48$ & $-18.28 \pm 0.55$ & $62.0 \pm 5.0$ & 69.0 & & B1npe & & & & 17 \\
\hline $81377^{*}$ & 149757 & $\zeta \mathrm{Oph}$ & 163709.53 & -103401.7 & $7.12 \pm 0.71$ & $13.07 \pm 0.85$ & $25.44 \pm 0.72$ & $-9.0 \pm 5.5$ & 23.5 & 348 & O9.5Vnn & 15.9 & 21.1 & 0.16 & 18 \\
\hline 102274 & 197911 & & 204321.62 & +63 1232.9 & $1.42 \pm 0.62$ & $-13.72 \pm 0.53$ & $-3.66 \pm 0.53$ & $-3.8 \pm \quad 5.0$ & 46.1 & & B5 & & & & 21 \\
\hline $109556^{*}$ & 210839 & $\lambda$ Cep & 221130.58 & +592452.3 & $1.98 \pm 0.46$ & $-7.22 \pm 0.44$ & $-11.06 \pm 0.39$ & $-75.1 \pm 1.2$ & 74.0 & 214 & O6I & 40.0 & 64.6 & $0.17^{l}$ & 22 \\
\hline & J0826+2637 & & 82651.31 & +263725.6 & 2.6 & $61 \pm 3$ & $-90 \pm 2$ & & & $=0.53$ & $\tau=4.92$ & $1.4^{p}$ & & & 1 \\
\hline & J0835-4510 & & 83520.68 & -451035.8 & 2.0 & $-48 \pm 2$ & $35 \pm 1$ & & & 0.09 & 0.01 & $1.4^{p}$ & & & 2 \\
\hline & $\mathrm{J} 1115+5030$ & & 111538.35 & +503013.6 & 1.9 & $22 \pm 3$ & $-51 \pm 3$ & & & 1.65 & 10.53 & $1.4^{p}$ & & & 4 \\
\hline & $\mathrm{J} 1932+1059$ & & 193213.87 & +105931.8 & 5.9 & $99 \pm 6$ & $39 \pm 4$ & & & 0.22 & 3.10 & $1.4^{p}$ & & & 8 \\
\hline & Geminga $^{q}$ & & 63354.15 & +174612.9 & $6.4 \pm 1.7$ & $138 \pm 4$ & $97 \pm 4$ & & & & & $1.4^{p}$ & & & 9 \\
\hline
\end{tabular}

Notes: $a: v_{\text {rot }} \sin i$ from Slettebak et al. (1997). $b$ : Total mass for the binary: B5V (5.8 $\left.M_{\odot}\right)+$ F8V (1.1 $\left.M_{\odot}\right)$. $c$ : $v_{\text {rad }}$ from Duflot et al. (1995). The Hipparcos Input Catalogue radial velocity is incorrect. $d: v_{\text {rot }} \sin i$ from Bernacca \& Perinotto (1970). e: We took the average $v_{\text {rad }}$ from Bohannan \& Garmany (1978), Garmany et al. (1980), Stone (1982), and Gies \& Bolton (1986). $f: v_{\text {rad }}$, i.e., center of mass velocity, from Stickland et al. (1987). $g$ : $v_{\text {rot }} \sin i$ from Gies (1987). $h$ : Spectral type of the secondary of $\iota$ Ori from Stickland et al. $i$ : Total mass for the binary $\iota$ Ori. The individual masses are $22.9 M_{\odot}$ for the primary and $14.9 M_{\odot}$ for the secondary. $j: v_{\text {rot }}$ sin $i$ from Morse et al. (1991). $k: v_{\text {rot }} \sin i$ from Uesugi \& Fukuda (1970). $l: \epsilon$ from Kudritzki \& Hummer (1990). $m$ : Assumed mass for main-sequence star instead of luminosity class IV. $n: v_{\text {rot }} \sin i$ from Brown \& Verschueren (1997). o: $v_{\text {rad }}$ from Gies \& Bolton (1986). p: We take the characteristic mass for a neutron star. $q$ : Position from Caraveo et al. (1998); parallax and proper motion from Caraveo et al. (1996). 
fraction $X=0.577$ of $\mathrm{H}$ ) and large rotational velocity $\left(348 \mathrm{~km} \mathrm{~s}^{-1}\right)$, and if the binary dissociated after the supernova explosion, we might be able to identify the associated neutron star. None of the pulsars in Fig. 2 was ever inside the Upper Centaurus Lupus subgroup, but two could have originated from the Upper Scorpius subgroup: PSR J1239+2453 and PSR J1932+1059 ${ }^{4}$.

We first consider PSR J1239+2453. Its estimated distance is $\sim 560 \mathrm{pc}$. It passed within about $20 \mathrm{pc}$ of the Upper Scorpius region $\sim 1$ Myr ago if and only if its (unknown) radial velocity is large and positive $\left(\sim 650 \mathrm{~km} \mathrm{~s}^{-1}\right)$. With a tangential velocity of $\sim 300 \mathrm{~km} \mathrm{~s}^{-1}$ (the proper motion is 114 mas $\mathrm{yr}^{-1}$ ), the space velocity would have to be over $700 \mathrm{~km} \mathrm{~s}^{-1}$, which is uncomfortably large. Furthermore, while $1 \mathrm{Myr}$ is consistent with the kinematic age for $\zeta \mathrm{Oph}$, it is in conflict with the characteristic age $(P /(2 \dot{P})=23 \mathrm{Myr})$ of the pulsar. The latter is an uncertain age indicator, but the difference between the two times is so large that we consider it unlikely that PSR J1239+2453 was associated with $\zeta$ Oph. The pulsar is currently at a Galactic latitude of $86^{\circ}$, i.e, at $z \sim 560$ pc above the Galactic plane. Typical $z$-oscillation periods of pulsars are of order 100 Myr (e.g., Blaauw \& Ramachandran 1998), so that maximum height is reached after $T_{1 / 4} \sim 25$ Myr. Taking the characteristic age at face value suggests the pulsar is near its maximum height above the plane, had a $z$-velocity of about $30 \mathrm{~km} \mathrm{~s}^{-1}$, and was not formed in the Upper Scorpius association (age $\sim 5 \mathrm{Myr}$ ), but was born $\sim 25 \mathrm{Myr}$ ago in the Galactic plane outside the Solar neighbourhood.

The path of the other pulsar, PSR J1932+1059 (earlier designation PSR B1929+10), also passed the Upper Scorpius association some 1-2 Myr ago. The characteristic age of this pulsar is only $\sim 3 \mathrm{Myr}$, consistent with the kinematic age of $\zeta \mathrm{Oph}$ within the uncertainties. The present $z$-velocity of the pulsar $\left(\sim 40 \mathrm{~km} \mathrm{~s}^{-1}\right.$ away from the Galactic plane) predicts a maximum distance away from the plane of $680 \mathrm{pc}$ and $T_{1 / 4} \sim 28 \mathrm{Myr}$. The pulsar is presently located only $\sim 10 \mathrm{pc}$ below the plane. Since it presumably formed close to the plane, this means that PSR J1932+1059 either formed recently or well over $50 \mathrm{Myr}$ ago. Considering that both the characteristic age and the typical pulsar ages (up to $~ 50 \mathrm{Myr}$ ) (Blaauw \& Ramachandran 1998) are significantly smaller than $\sim 50 \mathrm{Myr}$, we conclude that the pulsar formed recently. Upper Scorpius is the only site of star formation along the past trajectory of the pulsar. We thus consider PSR J1932+1059 a good candidate for the remnant of the supernova which caused the runaway nature of $\zeta$ Oph.

\subsection{Data}

Table 3 summarizes the data for $\zeta$ Oph and PSR $\mathrm{J} 1932+1059$. The radial velocity of the pulsar is unknown.

\footnotetext{
${ }^{4}$ Recently, Walter (2000) suggested that RX J185635-3754 as another candidate neutron star that could have encountered $\zeta$ Oph in the past. We show in Appendix B that this is unlikely.
}

The pulsar proper motion listed by Taylor et al. (1993) was calculated from timing measurements (Downs \& Reichley 1983). More accurate proper motions can be obtained from VLBI observations; Campbell (1995) measured a provisional proper motion and parallax of PSR J1932+1059 of $\left(\mu_{\alpha *}, \mu_{\delta}\right)=(96.7 \pm 1.7,41.3 \pm 3.5)$ mas $\mathrm{yr}^{-1}$ and $\pi=5 \pm 1.5$ mas, respectively, including a full covariance matrix. These measurements are in good agreement with those of Taylor et al. (1993; see Table 3 and Fig. 5).

\subsection{Simulations}

Our hypothesis is that $\zeta$ Oph and PSR J1932+1059 are the remains of a binary system in Upper Scorpius which became unbound when one of the components exploded as a supernova. Support for this hypothesis would be to find both objects at the same position at the same time in the past. Our approach is to calculate their past orbits and simultaneously determine the separation between the two objects, $D_{\min }(\tau)$, as a function of time, $\tau$. We define $D_{\min }(\tau)$ as $\mid \boldsymbol{x}_{\zeta}$ Oph $-\boldsymbol{x}_{\text {pulsar }} \mid$, where $\boldsymbol{x}_{j}$ is the position of object $j$. We consider the time $\tau_{0}$ at which $D_{\min }(\tau)$ reaches a minimum to be the kinematic age. To take the errors in the observables into account we calculate a large set of orbits, sampling the parameter space defined by the errors. We use the Taylor et al. (1993) proper motion for the pulsar. The errors in the positions of the runaway and the pulsar are negligible, and those in the proper motions of the two objects and in the parallax of the runaway are modest $(\leq 10 \%)$. However, the radialvelocity error of $\zeta \mathrm{Oph}$ is considerable $\left(5 \mathrm{~km} \mathrm{~s}^{-1}\right)$. The distance to the pulsar has a significant error, and its radial velocity is unknown. Accordingly, we first determine the region in the $\left(\pi_{\text {pulsar }}, v_{\text {rad,pulsar }}\right)$ parameter space for which the pulsar approaches the runaway when we retrace both orbits. Sampling a grid in $\left(\pi_{\text {pulsar }}, v_{\text {rad,pulsar }}\right)$ while keeping the other parameters fixed, we find that, for $2 \lesssim \pi_{\text {pulsar }} \lesssim 6$ mas and $100 \lesssim v_{\text {rad,pulsar }} \lesssim 300 \mathrm{~km} \mathrm{~s}^{-1}$, the motions of the pulsar and the runaway are such that their separation decreases as one goes back in time.

Adopting $\pi_{\text {pulsar }}=4 \pm 2$ mas and $v_{\text {rad,pulsar }}=200 \pm$ $50 \mathrm{~km} \mathrm{~s}^{-1}$, we calculate three million orbits for the pulsar and the runaway. Considering that the pulsar propermotion errors might be underestimated (Campbell et al. 1996; Hartman 1997), we increased them by a factor of two. For each run we create a set of positions and velocities for the runaway and the pulsar consistent with the (modified $3 \sigma$ ) errors on the observables. We also calculated the orbit of the Upper Scorpius association back in time, using the mean position and velocity derived by de Zeeuw et al. (1999, their Table 2). 30822 of these simulations resulted in a minimum separation between the pulsar and the runaway of less than 10 pc. In 4214 simulations the pulsar and the runaway had a minimum separation less than $10 \mathrm{pc}$ and were both situated within $10 \mathrm{pc}$ of the center of the association (the smallest minimum separation found was $0.35 \mathrm{pc})$. Thus, only a small fraction (0.14\%) of the 

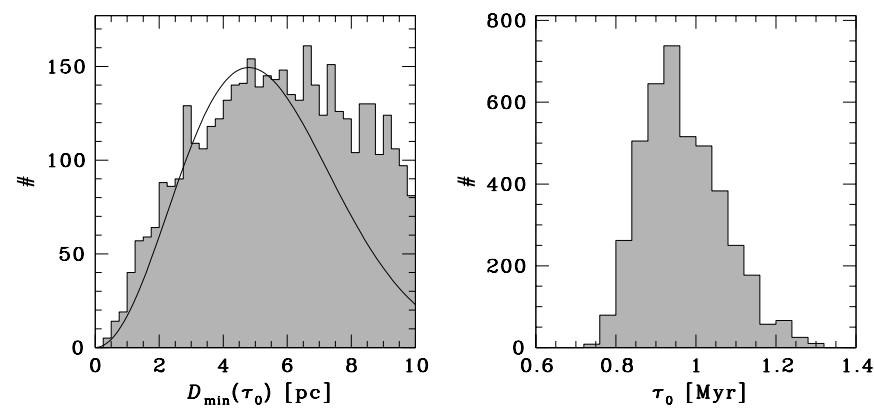

Fig. 3. Left: Distribution of minimum separations, $D_{\min }\left(\tau_{0}\right)$, between $\zeta$ Oph and PSR J1932+1059. The solid line denotes the expected distribution of $D_{\min }$, see Sect. 3.3. Right: Distribution of the times $\tau_{0}$ at which the minimum separation was reached

simulations is consistent with the hypothesis that the pulsar and the runaway were once, $\sim 1$ Myr ago, close together within the Upper Scorpius association. We now show that given the measurement uncertainties, this low fraction is perfectly consistent with the two objects being in one location in the past.

Figure 3 shows the distribution of the minimum separations, $D_{\min }\left(\tau_{0}\right)$, and the kinematic ages, $\tau_{0}$, of the 4214 simulations mentioned above. The lack of simulations which yield very small minimum absolute separations is due to the three-dimensional nature of the problem. Consider the following case: two objects are located at exactly the same position in space, e.g., the binary containing the pulsar progenitor and the runaway. However, the position measurement of each of these objects has an associated typical error. The distribution of the absolute separation between the objects, obtained from repeated measurements of the positions of both objects, can be calculated analytically for a Gaussian distribution of errors (see Appendix A). The solid line in Fig. 3 shows the result for an adopted distance measurement error of $2.5 \mathrm{pc}$, which agrees very well with our simulations. The peculiar statistical properties of the sample of successful simulations make it difficult to give a simple argument to derive the value of $2.5 \mathrm{pc}$ from the uncertainties in the kinematic properties of the runaway and the pulsar. We suspect that the disagreement between the solid line and histogram for separations $>6 \mathrm{pc}$ is most likely due to a slight mismatch between the model and the actual situation. Even so, Fig. 3 shows that due to measurement errors, very few simulations will produce a small observed minimum separation, even when the intrinsic separation is zero.

Figure 4 shows the astrometric parameters of the pulsar and the runaway at the start of the orbit integration, i.e., the "present" observables, for the simulations which result in a minimum separation less than $10 \mathrm{pc}$ occurring within Upper Scorpius. The parameters of $\zeta$ Oph show no correlations except between the parallax and the radial velocity. This is expected due to the degeneracy of these two quantities (a change in stellar distance, depending on whether it increases or decreases the separation between the star and the association, can be compensated for by a larger or smaller radial velocity, respectively).

The parameters of the pulsar behave very differently. In addition to the $\pi$ vs. $v_{\text {rad }}$ correlation, we find that the parallax is also correlated with both of the proper motion components. As a result, the proper motion components are correlated with each other. This means that only a subset of the full parameter space defined by the six-dimensional error ellipsoid of the pulsar fulfills the requirement that the pulsar and the runaway meet. Furthermore, if they met, then we know the radial velocity of the pulsar for each assumed value of its distance. A reliable distance determination would thus yield an astrometric radial velocity. The current best distance estimate of the pulsar derived from VLBI measurements, $\pi=5 \pm 1.5$ mas (Campbell 1995), predicts a radial velocity of $100-200 \mathrm{~km} \mathrm{~s}^{-1}$. This radial velocity is comparable to the tangential velocity: $\sim 100 \mathrm{~km} \mathrm{~s}^{-1}$ (for $\pi=5$ mas).

The pulsar proper motions of the 4214 successful simulations are shown in Fig. 5, together with the propermotion measurements of Lyne et al. (1982) (dot-dash line), Taylor et al. (1993) (solid line), and Campbell (1995) (dashed line). The measurements show a reasonable spread, reflecting the difficulty in obtaining pulsar proper motions, but are consistent, within $3 \sigma$, with the proper motions predicted by the simulations.

\subsection{Interpretation}

The observed astrometric and spectroscopic parameters of $\zeta$ Oph and PSR J1932+1059 are consistent with the assumption that these objects were very close together 1 Myr ago (Fig. 3). At that time both were within the boundary of Upper Scorpius (Fig. 6), which has a nuclear age of $\sim 5$ Myr (de Geus et al. 1989).

Several characteristics of $\zeta$ Oph and Upper Scorpius support the interpretation that the runaway and the pulsar must have been produced by the BSS. (i) The HI distribution in the direction of Upper Scorpius shows an expanding shell-like structure. De Geus (1992) argued that the energy output of the stellar winds of the massive stars in Upper Scorpius is two orders of magnitudes too low to account for the kinematics of the HI shell, and proposed that a supernova explosion created the Upper Scorpius HI shell. (ii) Based on the present-day mass function, de Geus showed that the initial population of Upper Scorpius most likely contained one star or binary more than the present population. The estimated mass of this additional object is $\sim 40 M_{\odot}$. Stars of this mass have main-sequence lifetimes of $\sim 4 \mathrm{Myr}$, and end their lives in a supernova explosion. Since the association has an age of $\sim 5 \mathrm{Myr}$, the supernova explosion might have taken place $\sim 1$ Myr ago. (iii) The characteristics of $\zeta \mathrm{Oph}$ are indicative of close binary evolution. The helium abundance is large, and the star has a large rotational velocity (see Sect. 1). These facts make it very likely that the same supernova event 

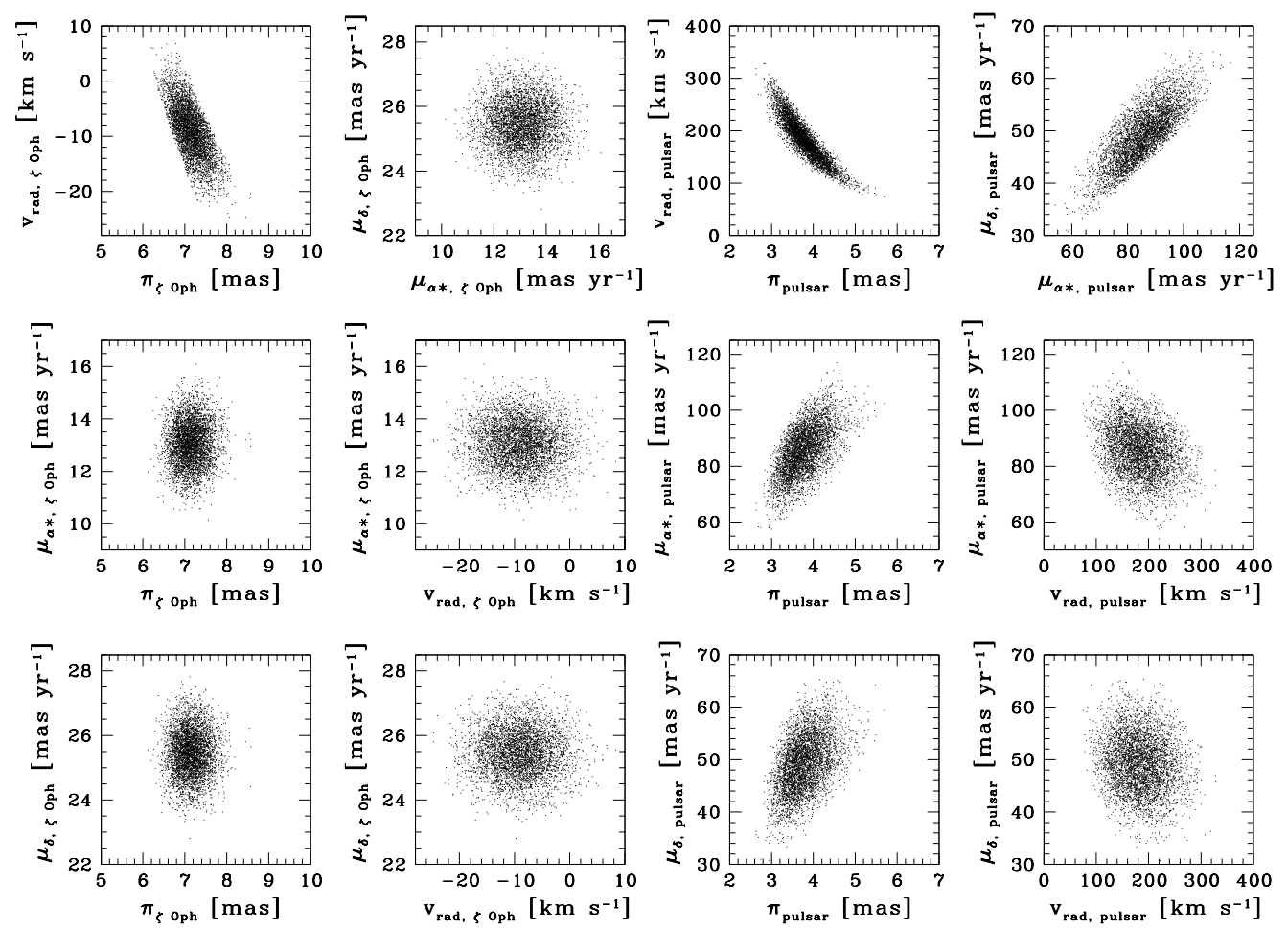

Fig. 4. Astrometric parameters of the runaway $\zeta$ Oph and the pulsar PSR J1932+1059 at the start of each of the 4214 simulations for which the minimum separation between the runaway and the pulsar was less than $10 \mathrm{pc}$, and both of them were within $10 \mathrm{pc}$ of the center of Upper Scorpius sometime in the past

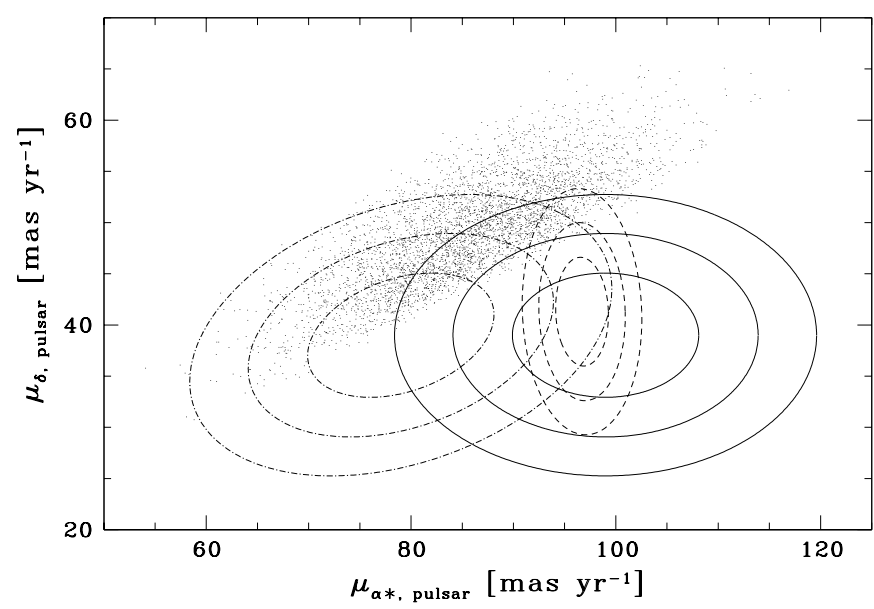

Fig. 5. Proper motions of the pulsar PSR J1932+1059 at the start of the 4214 successful simulations (dots; see Sect. 3.3), and the proper-motion measurements of the pulsar: dot-dash line denotes Lyne et al. (1982), solid line denotes Taylor et al. (1993), and the dashed line denotes Campbell (1995). The contours indicate the 1,2 , and $3 \sigma$ confidence levels

in Upper Scorpius created PSR J1932+1059 and endowed $\zeta$ Oph with its large velocity.

It could be that $\zeta$ Oph and PSR J1932+1059 are not related. This would imply that the pulsar originated in Upper Scorpius $\sim 1$ Myr ago and that $\zeta$ Oph obtained its large velocity either in a separate BSS event in Upper Scorpius, or in Upper Centaurus Lupus, $\sim 3 \mathrm{Myr}$ ago, as suggested by van Rensbergen et al. (1996). In the latter case, it would also have to be formed by the BSS, because of its high helium abundance, large rotational velocity, and the $\sim 10$ Myr difference between the age of Upper Centaurus Lupus and the kinematic age of $\zeta$ Oph (Sect. 1). Given the small probability of finding a runaway star and a pulsar with orbits that cross, and with both objects at the point of intersection at the same time, we conclude that $\zeta$ Oph and PSR J1932+1059 were once part of the same close binary in Upper Scorpius, providing the first direct evidence for the generation of a single runaway star by the BSS.

\subsection{Pulsar kick velocity}

If $\zeta$ Oph and PSR J1932+1059 were once part of a binary, then we can derive a number of properties of this system. For example, the true age of the pulsar must be the kinematic age of $1 \mathrm{Myr}$ (as compared to the characteristic age estimate of $3 \mathrm{Myr}$ ). It follows that, if no glitches occurred, the pulsar had a period of $0.18 \mathrm{~s}$ at birth, as compared to the current period of $0.22 \mathrm{~s}$.

The velocity distribution of the pulsar population is much broader (a few $\times 100 \mathrm{~km} \mathrm{~s}^{-1}$ ) than that of the pulsar progenitors $\left(\mathrm{a}\right.$ few $\left.\times 10 \mathrm{~km} \mathrm{~s}^{-1}\right)$. The mechanism responsible for this additional velocity (the "kick velocity" $\boldsymbol{v}_{\text {kick }}$ ) is not well understood (e.g., Lai 1999). The kick velocity is most likely due to asymmetries in the core of a star just before, or during, the supernova explosion.

The simulations described in Sect. 3.3 provide the velocities of the runaway star, the pulsar, and the 


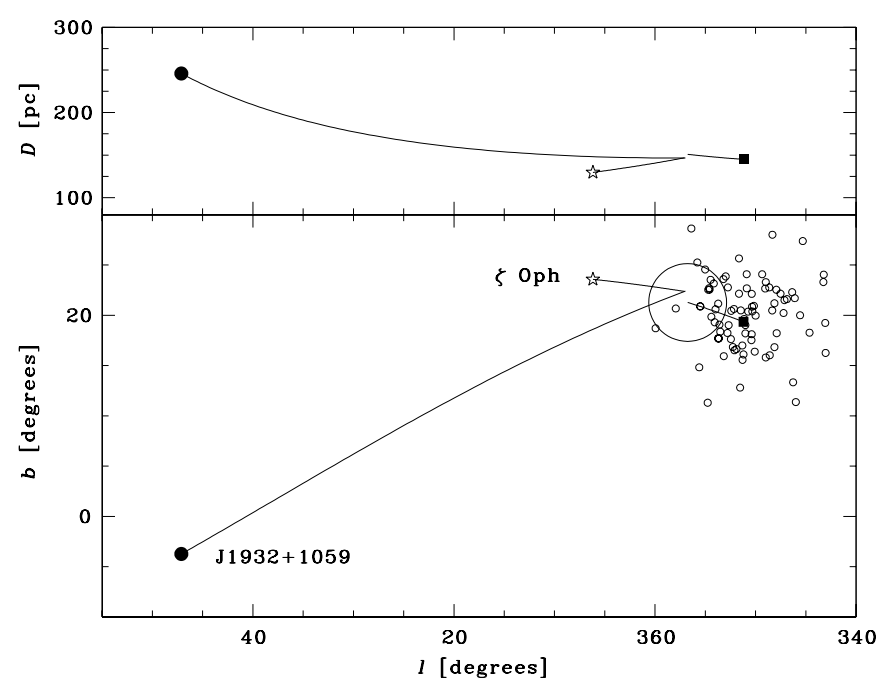

Fig. 6. The orbits of $\zeta$ Oph, PSR J1932+1059, and Upper Scorpius. The present positions are denoted by a star for the runaway, a filled circle for the pulsar, and by a filled square for the association. The top panel shows the distance vs. Galactic longitude of the stars. The bottom panel shows the orbits projected on the sky in Galactic coordinates. The small open circles in the bottom panel denote the present-day positions of the O, B, and A-type members of Upper Scorpius, taken from de Zeeuw et al. (1999). The large circle denotes the position of the association at the time of the supernova explosion, and has a $10 \mathrm{pc}$ radius. This figure assumes a set of space motions consistent with the common origin hypothesis

association at the present time. This makes it possible to determine $\boldsymbol{v}_{\text {kick }}$ of the neutron star. For the 4214 successful runs we find that the average velocities with respect to Upper Scorpius are: $\boldsymbol{v}_{\zeta}$ Oph $=(-6.4 \pm 4.2,33.8 \pm 1.4$, $5.8 \pm 2.0) \mathrm{km} \mathrm{s}^{-1}$ and $\boldsymbol{v}_{\text {pulsar }}=(48.6 \pm 21.7,222.9 \pm 36.1$, $-70.7 \pm 8.4) \mathrm{km} \mathrm{s}^{-1}$ in Galactic Cartesian coordinates $(U, V, W)^{5}$.

To derive $\boldsymbol{v}_{\text {kick }}$ we consider a binary with components of mass $M_{1}$ and $M_{2}$ in a circular orbit, in which the first component $\left(\right.$ star $\left._{1}\right)$ explodes as a supernova and creates a neutron star. At the time of the explosion, star $_{1}$ is the least massive component of the binary, due to the prior mass transfer phase, and is most likely a helium star. The rapidly expanding supernova shell, with mass $\Delta M=M_{1}-M_{\mathrm{n}}$, where $M_{\mathrm{n}}=1.4 M_{\odot}$ is the typical mass a neutron star, will quickly leave the binary system. The shell has a net velocity equal to the orbital velocity of $\operatorname{star}_{1}$ at the moment of the explosion $\left(v_{1}\right)$. A net amount of momentum $\left(\Delta M \times v_{1}\right)$ is thus extracted from the system and the binary reacts by moving in the opposite direction with a velocity $v=-\left(\Delta M \times v_{1}\right) /\left(M_{2}+M_{n}\right)$, the so-called "recoil velocity". The binary will remain bound after the explosion because less than half of the total mass of the system is expelled $\left(M_{1}<M_{2}\right.$; cf. Paper I). However, if the neutron star receives a kick in the supernova explosion

\footnotetext{
${ }^{5} U$ points in the direction of the Galactic centre, $V$ points in the direction of Galactic rotation, and $W$ points towards the North Galactic pole.
}

the binary might dissociate, depending on the direction and magnitude of the kick velocity. We simulate this by using a simple orbit integrator for two bodies. We determine the semi-major axis and orbital velocities assuming the binary has a circular orbit and masses $M_{1}=5 M_{\odot}$ and $M_{2}=15 M_{\odot}(\zeta \mathrm{Oph})$. We then change the mass of $\operatorname{star}_{1}$ to $M_{\mathrm{n}}$ and add a kick-velocity to its orbital velocity. We start the integration at this point and try to reproduce the observed velocity of $\zeta \mathrm{Oph}$, the pulsar, and the angle between the two velocity vectors $\left(35^{\circ}\right)$. It turns out that a kick velocity of order $350 \mathrm{~km} \mathrm{~s}^{-1}$ is needed in a direction almost opposite to the orbital velocity of star $_{1}$ 's prior to the explosion. This value is in good agreement with the average pulsar kick velocity found by Hartman (1997) and Hansen \& Phinney (1997). The current velocity of the pulsar, $\sim 240 \mathrm{~km} \mathrm{~s}^{-1}$, is more than $100 \mathrm{~km} \mathrm{~s}^{-1}$ smaller than the kick it acquired. Our simulations show that this deceleration is due to the gravitational pull of $\zeta$ Oph on the pulsar.

The mass of $\zeta$ Oph used in the above estimate is consistent with the calibration of Schmidt-Kaler (1982). The more recent mass calibration of Vanbeveren, Van Rensbergen \& De Loore (1998) suggests $21 M_{\odot}$ (Table 3 ). This would increase the inferred kick velocity to $\sim 400 \mathrm{~km} \mathrm{~s}^{-1}$.

\section{A dynamical ejection in Orion}

\subsection{AE Aurigae \& $\mu$ Columbae}

Blaauw \& Morgan (1954) drew attention to the isolated stars $\mathrm{AE}$ Aur $(\mathrm{O} 9.5 \mathrm{~V})$ and $\mu \mathrm{Col}(\mathrm{O} 9.5 \mathrm{~V} / \mathrm{B} 0 \mathrm{~V})$, which move away from the Orion star-forming region (e.g., McCaughrean \& Burkert 2000) in almost opposite directions with comparable space velocities of $\sim 100 \mathrm{~km} \mathrm{~s}^{-1}$ (Fig. 11, stars 5 and 6 in Table 3). Blaauw \& Morgan suggested that "... the stars were formed in the same physical process 2.6 million years ago and that this took place in the neighborhood of the Orion Nebula." The past orbits of $\mathrm{AE}$ Aur and $\mu \mathrm{Col}$ intersect on the sky near the location of the massive highly-eccentric double-lined spectroscopic binary $\iota$ Ori (O9III+B1III, see Stickland et al. 1987). This led Gies \& Bolton (1986) to suggest that the two runaways resulted from a dynamical interaction also involving $\iota$ Ori: “... ८ Ori is the surviving binary of a binary-binary collision that ejected both AE Aur and $\mu$ Col."

\subsection{Data}

Table 3 lists the data for AE Aur, $\mu$ Col, and $\iota$ Ori. We adopt Stickland's et al. (1987) radial velocity for $\iota$ Ori $\left(\boldsymbol{v}_{\gamma}\right)$. For the radial-velocity errors for AE Aur and $\mu \mathrm{Col}$ we use the largest errors quoted in either the Catalogue de Vitesses Radiales Moyennes Stellaires (Barbier-Brossat 1989), the Hipparcos Input Catalogue (Turon et al. 1992), the Wilson-Evans-Batten Catalogue (Duflot et al. 1995), or in the SIMBAD database. 

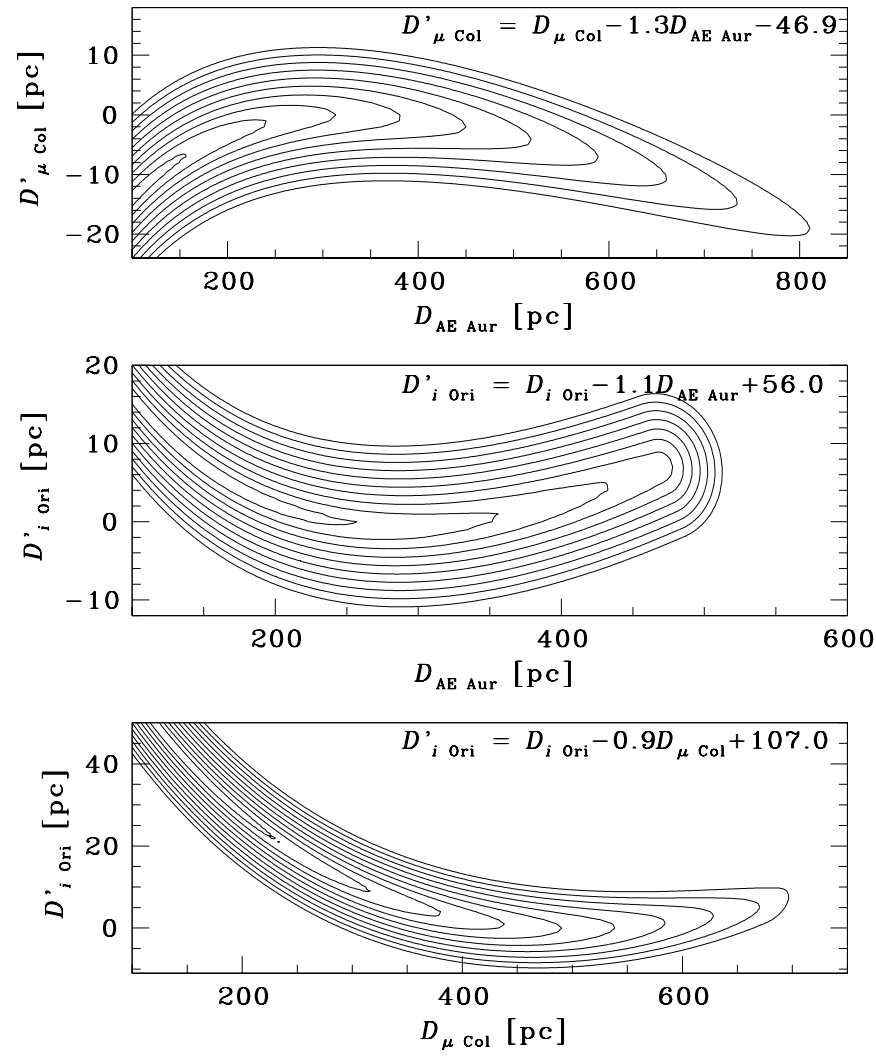

Fig. 7. Contours of minimum separation for the pairs AE Aur$\mu \mathrm{Col}($ top $)$, AE Aur $-\iota$ Ori (middle), and $\mu$ Col- $\iota$ Ori (bottom). The contours are spaced every $1 \mathrm{pc}$ with the outermost contour being $10 \mathrm{pc}$. The ordinates represent straight lines in the distance-distance plane, defined as indicated in the top right of each panel

\subsection{Simulations}

To investigate the hypothesis that the three stellar systems, AE Aur, $\mu \mathrm{Col}$, and $\iota$ Ori, were involved in a binarybinary encounter, we retrace their orbits back in time to find the minimum separation between them. As in Sect. 3, we explore the parameter space determined by the errors of, and correlations between, the observables.

Even with the unprecedented accuracy in trigonometric parallaxes obtained by the Hipparcos satellite, the errors on the individual distances are rather large: $D_{\mathrm{AE} \text { Aur }}=446_{-111}^{+220} \mathrm{pc}, D_{\mu \mathrm{Col}}=397_{-71}^{+110} \mathrm{pc}, D_{\iota}$ Ori $=$ $406_{-96}^{+185}$ pc. We therefore first determine which distances are most likely to agree with our hypothesis, and then study the effect of the measurement errors on the other observables. For each pair of stars, Fig. 7 shows contours of minimum separation between the respective orbits as a function of their present distances. The distances of the stars for which the orbits have a small minimum separation are strongly correlated, i.e., if the distance of star $i$ increases that of star $j$ also needs to increase to obtain a small minimum separation. We therefore choose to show the contours of constant minimum separation with respect to this correlation. The vertical axes thus show offsets from
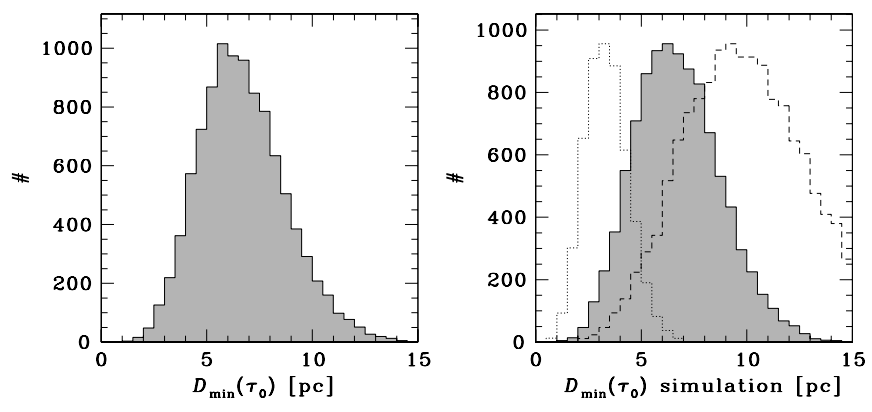

Fig. 8. Left: Distribution of minimum separations between AE Aur, $\mu$ Col, and $\iota$ Ori, $D_{\min }\left(\tau_{0}\right)$, of 10000 Monte Carlo simulations of the stellar encounter. Right: The $D_{\min }\left(\tau_{0}\right)$ distribution for three randomly drawn points from three spherical Gaussians with standard deviations of $\sigma=4 \mathrm{pc}$ (solid line and shaded), $\sigma=2 \mathrm{pc}$ (dotted line), and $\sigma=6 \mathrm{pc}$ (dashed line). The $D_{\min }\left(\tau_{0}\right)$ distribution for $\sigma=4 \mathrm{pc}$ is a good representation of the distribution in the left panel. Four pc is the typical spread in the end positions of the orbits due to the errors on the present day velocity $\left(\sim 2 \mathrm{~km} \mathrm{~s}^{-1}\right)$. The dotted and dashed histograms have been normalized such that their shapes can be compared with the solid histogram

the straight line in the distance vs. distance plane defined by the equation in the top right of each panel.

We start each simulation with a set of positions and velocities which are in agreement with the observed parameters and their covariance matrices $(<3 \sigma)$. Furthermore, we require the distances of the stars to fall within the $10 \mathrm{pc}$ minimum-separation contours of Fig. 7. We then calculate the orbits of $\mathrm{AE}$ Aur, $\mu \mathrm{Col}$, and $\iota$ Ori. We define the separation between the three stellar systems, $D_{\min }(\tau)$, as the maximum deviation of the objects from their average position, i.e., $D_{\min }(\tau)=\max \left|\boldsymbol{x}_{j}-\overline{\boldsymbol{x}}\right|$ for $j=\mathrm{AE}$ Aur, $\mu \mathrm{Col}$, and $\iota$ Ori, where $\overline{\boldsymbol{x}}=\frac{1}{3}\left(\boldsymbol{x}_{\mathrm{AE} \text { Aur }}+\boldsymbol{x}_{\mu \mathrm{Col}}+\boldsymbol{x}_{\iota \text { Ori }}\right)$ is the mean position and $\boldsymbol{x}_{j}$ the position of star $j$. The time $\tau_{0}$ at which $D_{\min }(\tau)$ reaches a minimum is considered to be the time of the encounter, i.e., the kinematic age.

We computed 2.5 million orbits, of which 114 yielded $D_{\min }\left(\tau_{0}\right)<1$ pc with $\tau_{0}=2-3 \mathrm{Myr}$. One of the simulations resulted in a minimum separation of $0.019 \mathrm{pc}$ which is equal to $4000 \mathrm{AU}$ (Fig. 8). The small number of simulations with small minimum separations is due to (i) the large number of parameters involved (i.e., 18) and (ii) the three-dimensional nature of the problem (cf. Sect. 3.3).

We have numerically determined the distribution of the minimum separations $D_{\min }$ of three points drawn from a three-dimensional Gaussian error distribution (the analytic results of Appendix A are valid only for two Gaussians). We randomly draw three points from three spherical three-dimensional Gaussians (with standard deviation $\sigma$ ) and determine $D_{\min }$. The Gaussians have the same mean positions. The resulting distribution for $\sigma=$ $4 \mathrm{pc}$ resembles the real one remarkably well (Fig. 8). A distance uncertainty of four pc is consistent with the $\sim 2 \mathrm{~km} \mathrm{~s}^{-1}$ uncertainties in the velocities of the runaways and $\iota$ Ori: $2 \mathrm{~km} \mathrm{~s}^{-1}$ over $\sim 2 \mathrm{Myr}$ results in a displacement 
of $\sim 4$ pc. Thus, the data and their errors are consistent with the hypothesis that $\sim 2.5 \mathrm{Myr}$ ago AE Aur, $\mu \mathrm{Col}$, and $\iota$ Ori were in the same small region of space.

\subsection{Interpretation}

The nominal observed properties of the runaway stars $\mathrm{AE}$ Aur and $\mu \mathrm{Col}$ and the binary $\iota$ Ori are consistent with a common origin $\sim 2.5$ Myr ago. The most likely mechanism that created the large velocities of the runaways and the high eccentricity of the $\iota$ Ori binary is a binary-binary encounter, as suggested by Gies \& Bolton (1986). The normal rotational velocities of both runaways $\left(25 \mathrm{~km} \mathrm{~s}^{-1}\right.$ and $111 \mathrm{~km} \mathrm{~s}^{-1}$ ) and the normal helium abundance of AE Aur (Table 3, see Blaauw 1993, Fig. 6) also suggest that these runaways were formed by the dynamical ejection scenario. The helium abundance of $\mu \mathrm{Col}$ is unknown.

\subsection{Parent cluster}

To find the cluster, or region of space, where the encounter between AE Aur, $\mu \mathrm{Col}$, and $\iota$ Ori took place we assume that the center of mass velocity of the three objects is identical to the mean velocity $\boldsymbol{v}_{\text {clus }}$ of the parent cluster. Then

$\boldsymbol{v}_{\mathrm{clus}}=\frac{\sum_{j} M_{j} \boldsymbol{v}_{j}}{\sum_{j} M_{j}}$,

for $j=\mathrm{AE}$ Aur, $\mu \mathrm{Col}$, and $\iota$ Ori. For each star we estimate the mass by interpolating the mass vs. spectraltype calibration of Schmidt-Kaler (1982, Table 23). We obtain $15.9 M_{\odot}$ for $\mathrm{AE}$ Aur and $\mu \mathrm{Col}$, and $22.9 M_{\odot}$ and $14.9 M_{\odot}$ for the primary and secondary of $\iota$ Ori, respectively $\left(37.8 M_{\odot}\right.$ for the binary system). We use the cluster velocity $\left(\boldsymbol{v}_{\text {clus }}\right)$ and the mean position of the three stellar systems at the moment of the encounter to integrate the orbit of the ensemble of stars $\tau_{0}$ Myr into the future. The position and velocity at the end of this integration should coincide with the present-day properties of the parent cluster. We extend the Monte Carlo simulations described in Sect. 4.3 to include the integration of the orbit of the "cluster" forward in time. Figures 9 and 10 summarize the results. Panel $b$ of Fig. 10 shows that the distances of the three stars and the predicted cluster distance are tightly correlated (see also Fig. 7); all distances increase when the cluster distance increases. A consequence of this tight correlation is that as soon as the distance to one of the objects is known, all other distances are fixed.

\subsubsection{Biases and measurement errors}

Two effects influence the mean cluster properties as predicted by the Monte Carlo simulations. First, it is easier to hit a target from close by than from far away, i.e., a larger range of velocities (within the errors) is consistent with the encounter hypothesis when the distance between the star and the encounter point is small (the "aiming effect"). We simulate this effect in the following way. We assume a range of cluster distances, 350-500 pc. For each distance we use Fig. 9 (the gray dots in the first row) to determine the other phase-space coordinates of the parent (position on the sky, proper motion, and radial velocity). With these "observables" we calculate the three-dimensional velocity of the cluster, corrected for Solar motion, and determine its position at a time $\tau_{0}$ (see first row in Fig. 9) in the past. We neglect the variation of the Galactic potential, ignore Galactic rotation, and use the linear velocity, to speed up the calculations. This past position of the cluster combined with the present three-dimensional positions of $\mathrm{AE}$ Aur, $\mu \mathrm{Col}$, and $\iota$ Ori (based on the present positions on the sky and the distances from Fig. 10 panel $b$ ) gives the velocities of the three stellar systems today, using $\tau_{0}$ as the time difference. These "observed" properties are then used as input for the Monte Carlo simulations described above to investigate the influence of the aiming effect on the predicted cluster distance. The circles in Fig. 10 panel $c$ display the bias in the cluster distance.

Secondly, the trigonometric distance of $\mu \mathrm{Col}$, $D_{\mu \text { Col }}=397_{-71}^{+110} \mathrm{pc}$, is smaller $(2 \sigma)$ than the observed photometric distance, $\sim 750$ pc (e.g., Gies 1987). The photometric distance is reliable since $\mu \mathrm{Col}$ is located in a region free of interstellar absorption. This difference between the trigonometric distance and the "real" distance results in an additional bias towards smaller distances for the stars and the cluster. In our Monte Carlo simulation we draw the parallaxes, like all other observables, from a Gaussian centred on the observed value and with a width equal to the observed error. For the Hipparcos distance of $\mu$ Col this means that less than $\sim 10 \%$ of the random realizations will be consistent with the photometric distance ${ }^{6}$. And because the distances of the three stellar systems and the cluster are correlated (see Fig. 10 panel $b$ ), the other stars also need to be at smaller distances for the encounter to take place. This effect will result in a mean cluster distance (the mean of the Monte Carlo simulations) which is underestimated. We simulated this effect in a similar manner as the aiming effect. The results on the mean cluster distance in the Monte Carlo simulations, aiming effect and the parallax of $\mu \mathrm{Col}$, are shown as the triangles in Fig. 10 panel $c$.

\subsubsection{Cluster properties and identification}

Taking the biases on the cluster distance into account, we reconstruct the present-day properties of the parent cluster of the stars AE Aur, $\mu \mathrm{Col}$, and $\iota$ Ori. The mean cluster distance from our Monte Carlo simulations is $339 \mathrm{pc}$ (right most panel in the second row of Fig. 9). The cluster

\footnotetext{
6 The Hipparcos parallax of $\mu \mathrm{Col}(2.52 \pm 0.55$ mas $)$ deviates more than $1.5 \sigma$ from the photometric parallax ( 1.3 mas). The random realisations of $\pi_{\mu}$ Col which are consistent with $\pi_{\text {phot }}$ thus need to fall outside the $-1.5 \sigma$ confidence level which occurs in fewer than $10 \%$ of the cases.
} 


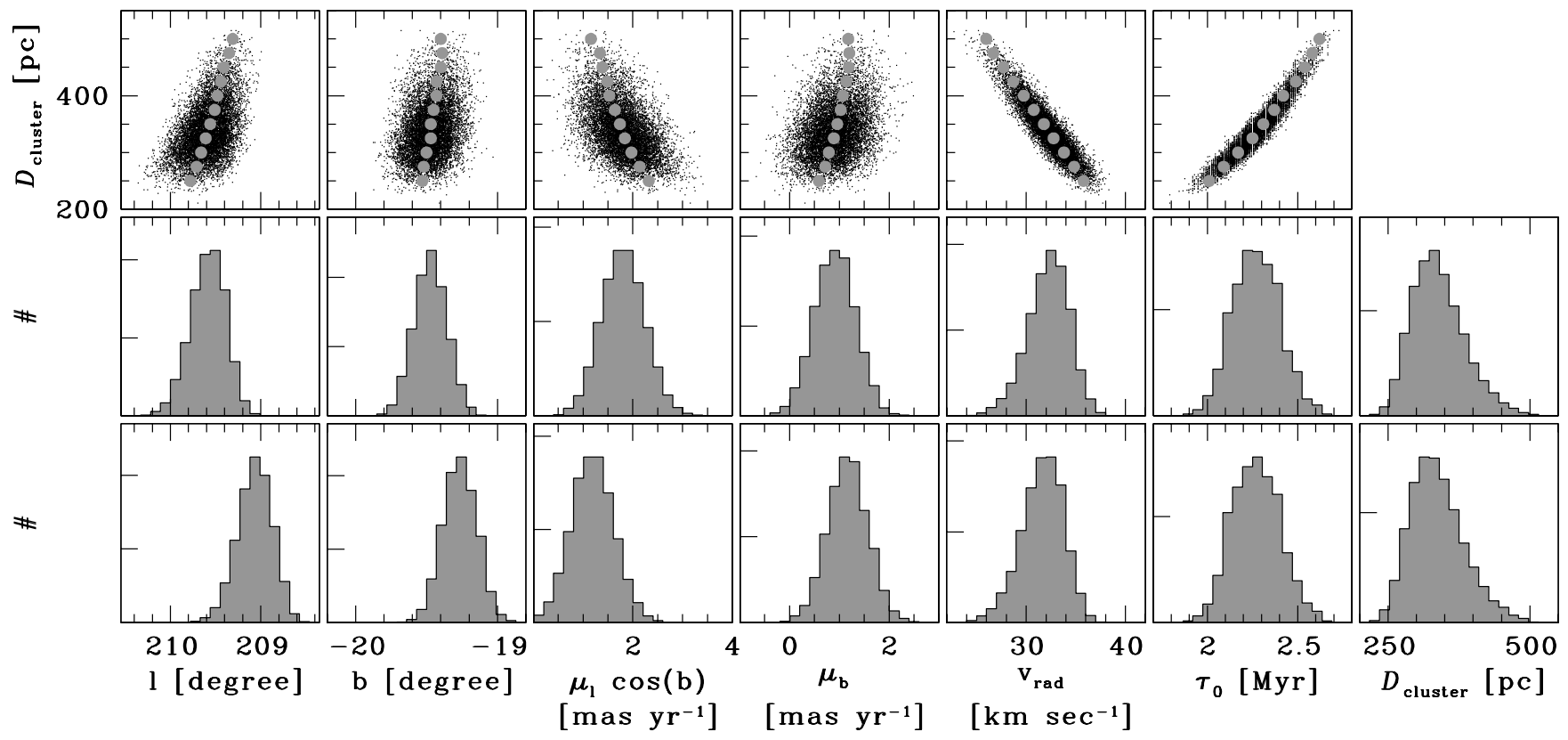

Fig. 9. Properties of the parent cluster of the runaways AE Aur and $\mu$ Col and the binary $\iota$ Ori obtained from our Monte Carlo simulations. First row: cluster properties plotted vs. the cluster distance. The grey dots denote the median values of the cluster properties for distance-bins of 25 pc. Second row: histograms of the predicted cluster properties. The tick marks on the vertical axis have a spacing of 1000. Third row: histograms of the predicted cluster properties when the mass of $\mu$ Col is changed by $-1 M_{\odot}$. Note that the distance, time, and radial-velocity histograms do not change significantly. The tick-mark spacing along the vertical axis is similar to that in the second row
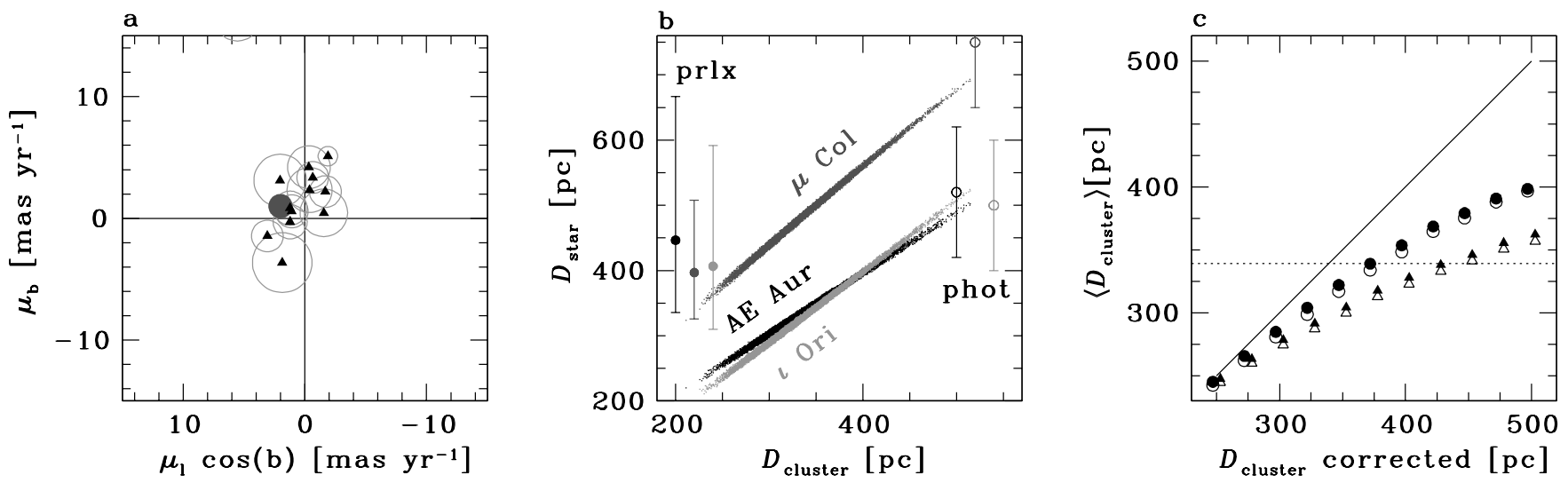

Fig. 10. Properties of the parent cluster of the runaways AE Aur and $\mu$ Col and the binary $\iota$ Ori. a) Proper motions and their errors (grey circles) for all stars in the Tycho 2 Catalogue (filled triangles) within an area of 0.4 by 0.4 centred on the Trapezium cluster. The large grey dot denotes the average of the predicted cluster proper motion for the Monte Carlo simulations. b) Distances of the runaway stars as a function of the cluster distance in the Monte Carlo simulations. The different grey scales are labeled in the panel. The filled circles and their error bars denote the observed distances of the stars derived from the Hipparcos parallaxes (prlx) and the open circles denote the distances derived from photometry (phot) (Gies 1987). c) the biases on the predicted cluster distance as discussed in Sect. 4.5.1. The filled and open symbols denote the mean and median, respectively, of the cluster distance distributions. The circles include only the first bias, the aiming effect, and the triangles include both the aiming effect and the "incorrect" Hipparcos parallax of $\mu$ Col. For clarity, the circles and triangles are displaced -3 and $3 \mathrm{pc}$, respectively. The dotted line indicates the mean cluster distance based on the Monte Carlo simulation

distance corrected for biases is $425-450$ pc. Using this distance we determine the other properties of the cluster (first row of Fig. 9), and summarize them in Table 4. Figure 9 (right most panel of the first row) indicates that the encounter happened 2.5 Myr ago; this obviously is a lower limit to the age of the cluster. Figure 11 shows the region of the sky where the parent cluster should be located: the Orion Nebula. The black contours in the bottom panel show the distribution of $(\ell, b)$ of the parent cluster obtained from the Monte Carlo simulations. 
Table 4. Predicted properties of the parent cluster of the stellar systems $\mathrm{AE} \mathrm{Aur,} \mu \mathrm{Col}$, and $\iota$ Ori: the cluster distance $D_{\text {parent }}$, the sky position in equatorial $(\alpha, \delta)$ and Galactic $(\ell, b)$ coordinates, the proper motions $\left(\mu_{\alpha *}, \mu_{\delta}\right)$ and $\left(\mu_{\ell *}, \mu_{b}\right)$, and the radial velocity $v_{\text {rad }}$. The predicted distances of the runaways and $\iota$ Ori if there was an encounter: $D_{\mathrm{AE}}$ Aur $=430 \mathrm{pc}$, $D_{\mu \text { Col }}=600 \mathrm{pc}$, and $D_{\iota}$ Ori $=440 \mathrm{pc}$

\begin{tabular}{lrrl}
\hline \hline & & $M_{\mu \mathrm{Col}}-1 M_{\odot}$ & \\
\hline$D_{\text {parent }}$ & $425-450$ & $425-450$ & $\mathrm{pc}$ \\
$(\alpha, \delta)$ & $\left(84^{\circ} .0,-5.8\right)$ & $\left(83^{\circ} .9,-5.2\right)$ & \\
$\left(\mu_{\alpha *}, \mu_{\delta}\right)$ & $(1.7,-0.8)$ & $(1.7,-0.2)$ & $\mathrm{mas} \mathrm{yr}^{-1}$ \\
$(\ell, b)$ & $\left(209^{\circ} 4,-19^{\circ} .4\right)$ & $\left(208^{\circ} .9,-19.2\right)$ & \\
$\left(\mu_{\ell *}, \mu_{b}\right)$ & $(1.3,1.2)$ & $(0.9,1.4)$ & $\mathrm{mas} \mathrm{yr}^{-1}$ \\
$v_{\text {rad }}$ & 28.3 & 27.6 & $\mathrm{~km} \mathrm{~s}^{-1}$ \\
\hline \hline
\end{tabular}

Of all clusters in this active star-forming region the Trapezium cluster (NGC 1976) is the most likely parent cluster for the following reasons.

1: The cluster is young. Palla \& Stahler (1999) find a mean age of $\sim 2$ Myr based on theoretical pre-mainsequence tracks, and established that the first stars formed not more than 5 Myr ago. Thus, the Trapezium is old enough to have produced the runaways;

2: The Trapezium is one of the most massive, dense clusters in the Solar neighbourhood. Estimates for the stellar density are $>20000$ stars $\mathrm{pc}^{-3}$ for the inner 0.1-0.3 pc (e.g., McCaughrean \& Stauffer 1994; Hillenbrand \& Hartmann 1998). These high stellar densities favor dynamical interactions within the cluster core;

3: The Trapezium shows a strong mass segregation (Zinnecker et al. 1993; Hillenbrand \& Hartmann 1998). Five of the six stars more massive than $10 M_{\odot}$ are in the centre. This concentration of massive stars increases the probability for dynamical interactions between these stars;

4: The binary fraction in the Trapezium cluster is at least as high as that of the Solar-type field stars, i.e., $\sim 60 \%$ (Prosser et al. 1994; Petr et al. 1998; Simon et al. 1999; Weigelt et al. 1999). This means that enough binary systems are available for binary-binary or binarysingle-star interactions to become efficient in expelling stars from the cluster.

The mean astrometric properties and the radial velocity of the Trapezium agree perfectly with those predicted by our Monte Carlo simulation. The distance to the Trapezium is estimated to be $450-500$ pc (Walker 1969; Warren \& Hesser 1977a, 1977b, 1978; Genzel \& Stutzki 1989); we predict $425-450 \mathrm{pc}$. The observed radial velocity of the Trapezium is $23-25 \mathrm{~km} \mathrm{~s}^{-1}$ (Johnson 1965; Warren \& Hesser 1977a, 1977b; Abt et al. 1991; Morrell \& Levato 1991); we predict $\sim 28 \mathrm{~km} \mathrm{~s}^{-1}$. The absolute proper motion of the Trapezium is ill-determined, but is known to be small (e.g., de Zeeuw et al. 1999). We collected all stars, within a 0.4 by 0.4 region centred on the Trapezium, based on the Tycho 2 Catalogue (Høg 2000), and plot the proper motions in Fig. 10a. The proper motions agree with the predicted cluster proper motion.

Table 4 shows that the predicted position on the sky of the parent cluster does not fully agree with the position of the Trapezium (see Fig. 11). Here it is important to remember that we did not allow for any errors on the stellar masses used in Eq. (2). We investigate the effect of mass errors by changing the masses and running a new set of Monte Carlo simulations. We find that (i) the results are insensitive to the mass of $\iota$ Ori: a change as large as $\pm 5 M_{\odot}$ produces no noticeable change in the cluster properties, and (ii) the sky position of the parent cluster and its proper motion depend on the mass ratio of AE Aur and $\mu$ Col. Changing the mass of $\mu$ Col by $-1 M_{\odot}$ or the mass of AE Aur by $+1 M_{\odot}$ shifts the predicted sky position of the parent cluster to that of the Trapezium cluster (Fig. 11). A mass change in the other direction, $+1 M_{\odot}$ for $\mu \mathrm{Col}$ and $-1 M_{\odot}$ for AE Aur, creates a similar shift in the opposite direction. There are indications from spectral-type determinations that $\mu \mathrm{Col}$ is indeed slightly less massive than AE Aur. Most spectral-type determinations of $\mu$ Col give $09.5 \mathrm{~V}$; however, Blaauw \& Morgan (1954) and Paper I quote B0V and Houk (1982) quotes $\mathrm{B} 1 \mathrm{IV} / \mathrm{V}$.

We note that the calibration of Vanbeveren et al. (1998) gives a mass of $38.6 M_{\odot}$ for $\iota$ Ori, similar to that found with the Schmidt-Kaler calibration, but increases the masses of AE Aur and $\mu \mathrm{Col}$ to $21.1 M_{\odot}$. This does not change our results, as it is the ratio of the runaway masses that determines the predicted current position of the parent cluster.

In summary, the position, distance, proper motion, and radial velocity of the Trapezium cluster fall within the range predicted by our Monte Carlo simulations. Furthermore, the youth, extreme stellar density, mass segregation, and the high binary fraction make it the best candidate for the parent cluster of the runaways AE Aur and $\mu \mathrm{Col}$ and the binary $\iota$ Ori. Finally, it is the only likely candidate in this region of the sky.

\section{53 Ari, $\xi$ Per, $\zeta$ Pup, and $\lambda$ Cep}

The previous sections gave a specific example of each formation mechanism for runaway stars, and described our orbit retracing methods in detail. We now consider the three other classical runaways, as well as $\zeta$ Pup, and we discuss the likely formation mechanisms. The results are summarized in Table 5.

\subsection{Arietis \& Orion OB1}

(star 2)

Blaauw (1956) classified 53 Arietis (HIP 14514) as a runaway star based on its proper motion which is directed away from the Orion association. He deduced that 53 Ari left the Orion association $\sim 4.8 \mathrm{Myr}$ ago and predicted the radial velocity of the star (at that time unknown) to be $\sim 18 \mathrm{~km} \mathrm{~s}^{-1}$. The discrepancy in kinematic ages 

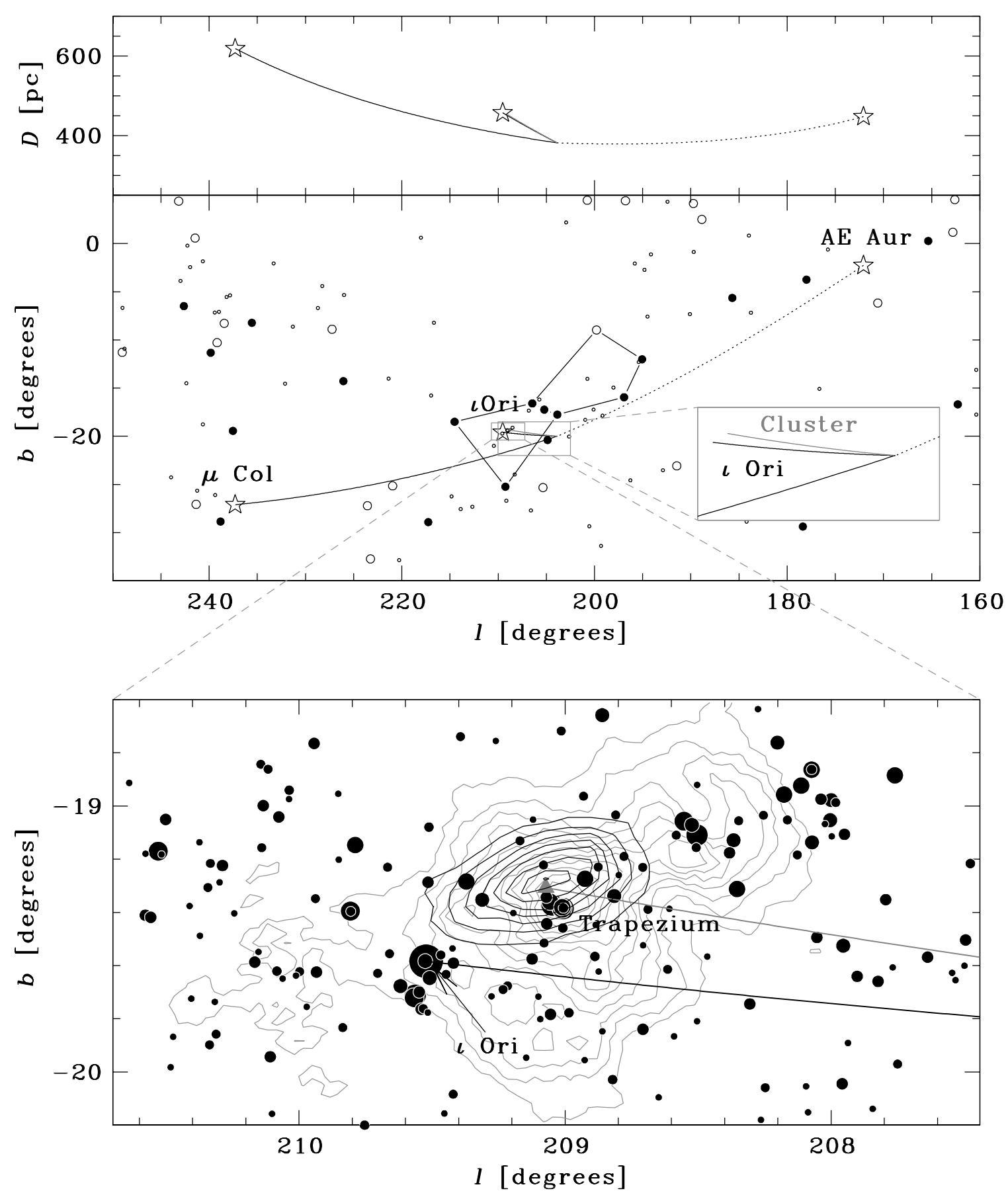

Fig. 11. Top \& middle: Orbits, calculated back in time, of the runaways AE Aur (dotted line) and $\mu$ Col (solid line) and the binary $\iota$ Ori for one of the Monte Carlo simulations described in the text. The top panel shows the distance vs. Galactic longitude of the stars. The middle panel shows the orbits projected on the sky in Galactic coordinates. The starred symbols depict the present position of the three stars. The stars met $\sim 2.5$ Myr ago. Using conservation of linear momentum, the orbit of the parent cluster (grey solid line, see blow up) is calculated from the time of the assumed encounter to the present. The large circles denote all stars in the Hipparcos Catalogue brighter than $V=3.5$ mag; filled circles denote O and B stars, open circles denote stars of other spectral type. The small circles denote the $\mathrm{O}$ and B type stars with $3.5 \mathrm{mag} \leq V \leq 5 \mathrm{mag}$ (cf. Fig. 1 in Blaauw \& Morgan 1954). The Orion constellation is indicated for reference. Bottom: The predicted position of the parent cluster (black contours) together with all stars in the Tycho Catalogue (ESA 1997) in the field down to $V=12.4$ mag. The size of the symbols scales with magnitude; the brightest star is $\iota$ Ori. The Trapezium and $\iota$ Ori are indicated. The black and dark grey lines are the past orbits of $\iota$ Ori and the Trapezium, respectively (see top panel). The triangle denotes the predicted present-day position of the parent cluster for this particular simulation. The grey contours display the IRAS 100 micron flux map, and mainly outline the Orion Nebula 

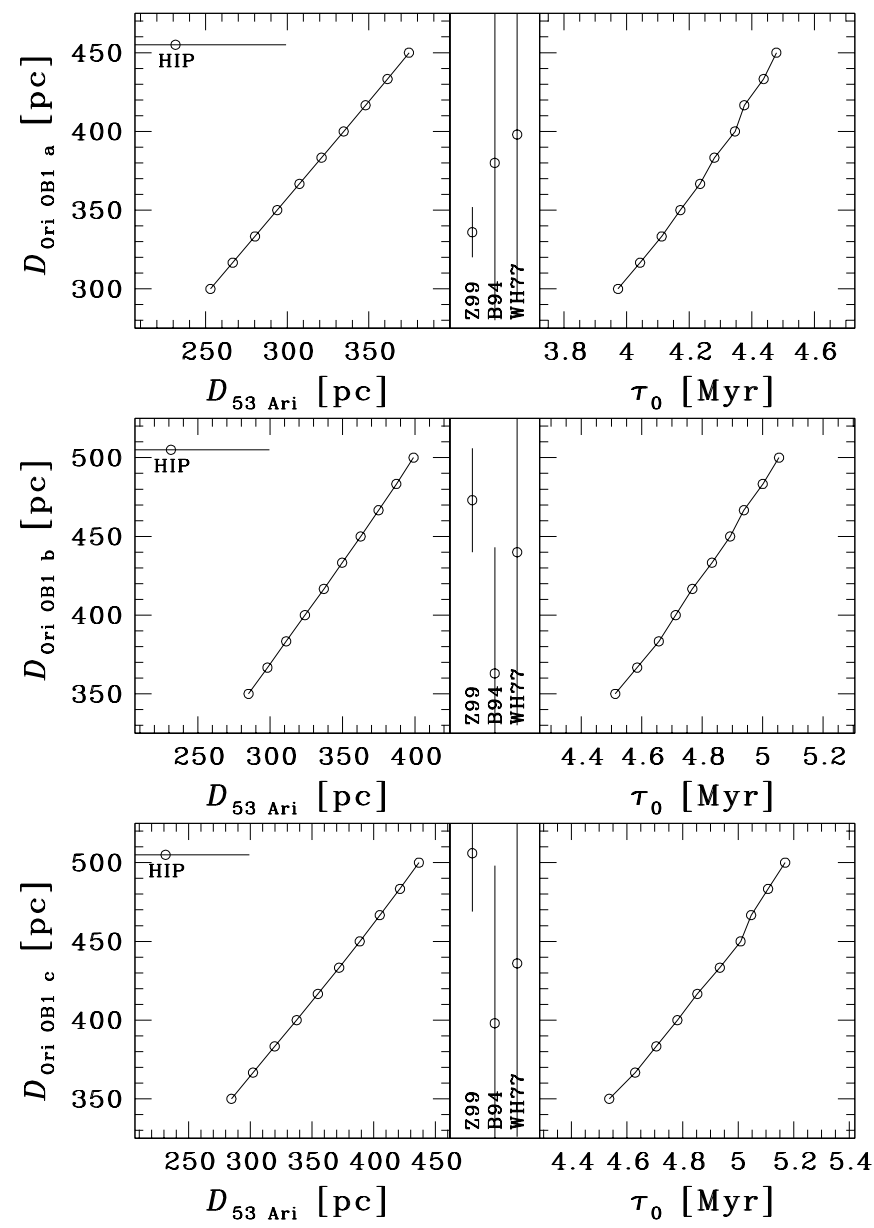

Fig. 12. Predicted distance (left) and kinematic age (right) of 53 Arietis as a function of the distance of the parent association: Ori OB1 subgroup $a$ (top), Ori OB1 subgroup $b$ (middle), and Ori OB1 subgroup $c$ (bottom). The Hipparcos distance and its $1 \sigma$ error are indicated in the left panels. Distance estimated by Warren \& Hesser (1977a, 1977b, WH77), Brown et al. (1994, B94), and de Zeeuw et al. (1999, Z99) are indicated in the middle panels

of $\mathrm{AE}$ Aur and $\mu \mathrm{Col}$ (Sect. 4) and that of 53 Ari indicates that it is not related to the same event that created $\mathrm{AE}$ Aur and $\mu \mathrm{Col}$ (Sect. 4) but is another runaway from the Orion star-forming region. Table 3 lists the observables of $53 \mathrm{Ari}^{7}$.

The Ori OB1 association has four subgroups: $a, b, c$, and $d$ (Blaauw 1964; Brown et al. 1994). We do not consider subgroup $d$ (the Trapezium) as a possible parent group of 53 Ari, since this subgroup is younger than the runaway (Sect. 4). The ages of the other subgroups are: 8-12 Myr for subgroup $a, 2-5$ Myr for subgroup $b$, and

\footnotetext{
7 The Hipparcos Input Catalogue (Turon et al. 1992) lists the radial velocity from the Catalogue de Vitesses Radiales Moyennes Stellaires (Barbier-Brossat 1989) which is incorrect $\left(-8.5 \mathrm{~km} \mathrm{~s}^{-1}\right)$. The radial velocity in the Troisième Catalogue Bibliographique de Vitesses Radiales Stellaires (Barbier-Brossat et al. 1994) is also incorrect. They list a radial velocity corrected for Solar motion of $15.3 \mathrm{~km} \mathrm{~s}^{-1}$ adopted from Sterken (1988). The uncorrected radial velocity is $24.2 \mathrm{~km} \mathrm{~s}^{-1}$.
}

〜4 Myr for subgroup $c$ (Warren \& Hesser 1977a, 1977b; Brown et al. 1994).

\section{Simulations}

We performed a set of simulations as in Sect. 2, retracing orbits for each subgroup $(a, b, c)$. The kinematic age of 53 Ari from subgroup $a$ is $\sim 4.3 \mathrm{Myr}$ (Fig. 12). This means that the subgroup was $\sim 6 \mathrm{Myr}$ old when 53 Ari became a runaway star. This very likely rules out the DES as the formation mechanism (see Sect. 1). However, there is little direct evidence in favor of the BSS. The helium abundance of 53 Ari is unknown and its observed rotational velocity is small $\left(v_{\text {rot }} \sin i=10 \mathrm{~km} \mathrm{~s}^{-1}\right)$, but this could be caused by a near pole-on orientation. We did not find a neutron star associated with 53 Ari, but our sampling of the nearby compact objects is severely limited (Sect. 2.1).

If subgroup $b$ is the parent association the kinematic age for 53 Ari is $\sim 4.8 \mathrm{Myr}$. This is comparable to the canonical age of the subgroup, and excludes the BSS as a production mechanism for 53 Ari (see Sect. 1). If Ori OB1 $b$ is the parent group of 53 Ari then the kinematic age is $\sim 4.8 \mathrm{Myr}$ and the formation mechanism is most likely the DES. However, the most recent age determination (Brown et al. 1994) gives $1.7 \pm 1.1 \mathrm{Myr}$. If Ori OB1 $b$ is indeed this young then the subgroup is younger than 53 Ari and cannot be the parent group.

For subgroup $c$ we find that the minimum separation between the subgroup centre and the runaway was never smaller than $15 \mathrm{pc}$, while the simulations for the other two subgroups $a$ and $b$ yield minimum separations as small as 1 pc. The space motion of Ori OB1 is mostly directed radially away from the Sun, and the proper motion component is relatively small. The Hipparcos data did not allow de Zeeuw et al. (1999) to discriminate between the different subgroups in their selection procedure; they only give one proper motion and radial velocity for the whole Orion complex. It is possible that subgroup $c$ has a motion that differs slightly from that of the other two subgroups, so that it cannot be ruled out as a candidate parent group. The age of subgroup $c, \sim 5 \mathrm{Myr}$, is similar to the kinematic age of 53 Ari. By the argument given above this suggests that if Ori OB1 $c$ is the parent association of 53 Ari, then the formation mechanism is most likely the DES.

In order to decide which of the Ori OB1 subgroups is the parent group of $53 \mathrm{Ari}$, we need to know the distances and velocities of the subgroups and the runaway star with a better accuracy than is now available. Figure 12 could then be used to pin down the parent group, and the mechanism which is responsible for the runaway nature of 53 Ari. Since subgroup $a$ is the only one for which the BSS is indicated, finding a pulsar originating from subgroup $a$ at the same time as 53 Ari would also clinch the issue. 


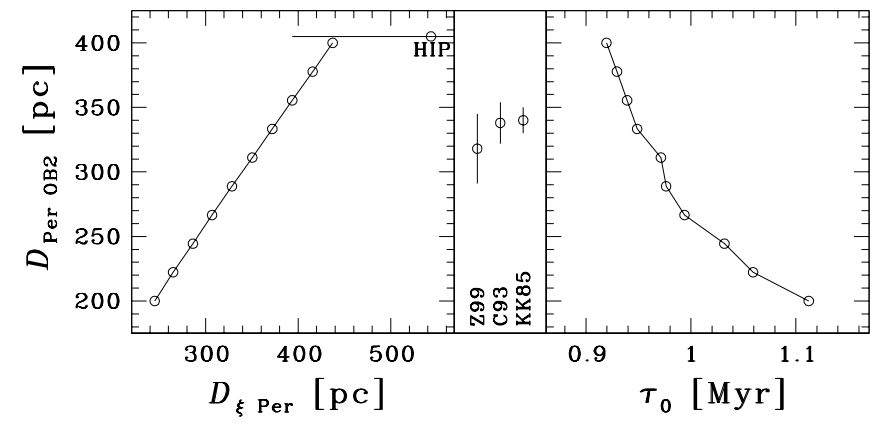

Fig. 13. Predicted distance (left) and kinematic age (right) of $\xi$ Per as a function of the distance of Per OB2. The Hipparcos distance and its $1 \sigma$ error are indicated in the left panel. Distance estimates for Per OB2 by Klochkova \& Kopylov (1985, KK85), Černis (1993, C93), and de Zeeuw et al. (1999, Z99) are indicated in the middle panel

\section{2. $\xi$ Persei \& Perseus OB2}

(star 3)

The O7.5III star $\xi$ Persei (HIP 18614) lies within the boundary of the Per OB2 association on the sky. This positional coincidence, and the low density of early-type stars near Per OB2, led Blaauw (1944) to propose $\xi$ Per as a member of the association. At that time it was thought that the large radial-velocity difference between $\xi$ Per and Per OB2 $\left(\sim 40 \mathrm{~km} \mathrm{~s}^{-1}\right)$ was due to uncertainties in the measurement of $v_{\text {rad }}$ for $\xi$ Per. However, when the radial velocity of $\xi$ Per was confirmed, its membership of the Per OB2 association became doubtful (Blaauw 1952a). In Paper I, Blaauw classified $\xi$ Per as a runaway star from the Per OB2 association, which naturally explains the discrepancy of the radial velocity of the star and the association. $\xi$ Per was the first star to be classified as runaway based on its $v_{\text {rad }}$ alone. Most other runaways were recognized because their proper motions were directed away from an association. The parent group, Per OB2, has an age of $\sim 7$ Myr (e.g., Seyfert et al. 1960; de Zeeuw \& Brand 1985).

\section{Data}

We adopt $v_{\text {rad }}=58.8 \mathrm{~km} \mathrm{~s}^{-1}$ for $\xi$ Per (Bohannan \& Garmany 1978; Garmany et al. 1980; Stone 1982; Gies \& Bolton 1986). This value differs by $10 \mathrm{~km} \mathrm{~s}^{-1}$ from those quoted in the Hipparcos Input Catalogue $\left(67.1 \mathrm{~km} \mathrm{~s}^{-1}\right.$, Turon et al. 1992) and the WEB catalogue $\left(70.1 \mathrm{~km} \mathrm{~s}^{-1}\right.$, Duflot et al. 1995), which derive from the value listed in the General Catalogue of Radial Velocities $\left(70.1 \mathrm{~km} \mathrm{~s}^{-1}\right.$, Wilson 1953). We take the radial-velocity error to be $5 \mathrm{~km} \mathrm{~s}^{-1}$; this is equal to the amplitude of the velocity variations induced by the non-radial pulsations of $\xi$ Per (de Jong et al. 1999). The rotational velocity and helium abundance are $v_{\text {rot }} \sin i=200 \mathrm{~km} \mathrm{~s}^{-1}$ and $\epsilon=0.18$, respectively (see also Table 3 ).

\section{Simulations}

Our orbit calculations (Sect. 2) show that the kinematic age of $\xi$ Per is $\sim 1$ Myr (Fig. 13). At that time the star was located $\sim 5$ pc from the center of Per OB2, well inside the association. Figure 13 also shows that the present distance of the runaway is $360 \mathrm{pc}$, assuming $318 \mathrm{pc}$ as the distance of Per OB2 (de Zeeuw et al. 1999). This distance for $\xi$ Per is consistent with the Hipparcos parallax at the $2 \sigma$ level.

We infer that the BSS is responsible for the runaway nature of $\xi$ Per based on (i) the 6 Myr age of Per OB2 at the time that $\xi$ Per was ejected, (ii) the high helium abundance of $\xi$ Per, (iii) its blue straggler nature (Sect. 9), and (iv) the large rotational velocity (see Sect. 1). Further evidence of a supernova explosion in the Per OB2 association is provided by a shell structure containing HI, dust, OH, CH, and other molecules (Sancisi 1970; Sancisi et al. 1974). This feature has been interpreted as a supernova shell which is physically connected to the Per OB2 association. We have not found a pulsar counterpart.

$\xi$ Per presently illuminates the California Nebula (NGC 1499), resulting in an HII emission region. The distance of this nebula is hard to determine (350-525 pc; Bohnenstengel \& Wendker 1976; Sargent 1979; Klochkova \& Kopylov 1985; Shull \& van Steenberg 1985), but must be similar to that of $\xi$ Per, i.e., $\sim 360$ pc.

\section{3. $\zeta$ Puppis, Vela R2, Vela OB2 \& Trumpler 10}

(star 10)

The O4I star $\zeta$ Puppis (HIP 39429) is the brightest and nearest single $\mathrm{O}$ star to the Sun. Its location outside any known association and its large space velocity $\left(\sim 60 \mathrm{~km} \mathrm{~s}^{-1}\right)$ led Upton (1971) to propose $\zeta$ Pup as a runaway star. In spite of the many groups of young, massive stars in the direction of $\zeta$ Pup, Upton could not make a unique identification of the parent association. He proposed Vel OB2 as the most likely candidate. In a recent study of the stars and ISM in the direction of Vela, Sahu (1992) proposed the $\mathrm{R}$ association Vel R2 as a possible parent for $\zeta$ Pup, based on the retraced path of the runaway on the sky. For our simulations we considered Vel OB2, Vel R2, the open cluster NGC 2391, and the cluster/association Tr 10 (de Zeeuw et al. 1999) which also lies in the same direction as Vel R2.

\section{Data}

Table 3 summarizes the data for $\zeta$ Pup. The position and velocity for the associations Vel OB2 and Tr 10 are adopted from de Zeeuw et al. (1999). Only two of the Vel R2 members are contained in the Hipparcos Catalogue. Their distances, $411_{-143}^{+473}$ (HIP 43792) and $294_{-61}^{+107}$ (HIP 43955), have large uncertainties or do not agree well with the canonical distance of Vel R2, $\sim 870$ pc (Herbst 1975). We are therefore unable to obtain meaningful phase-space coordinates of Vel R2. 


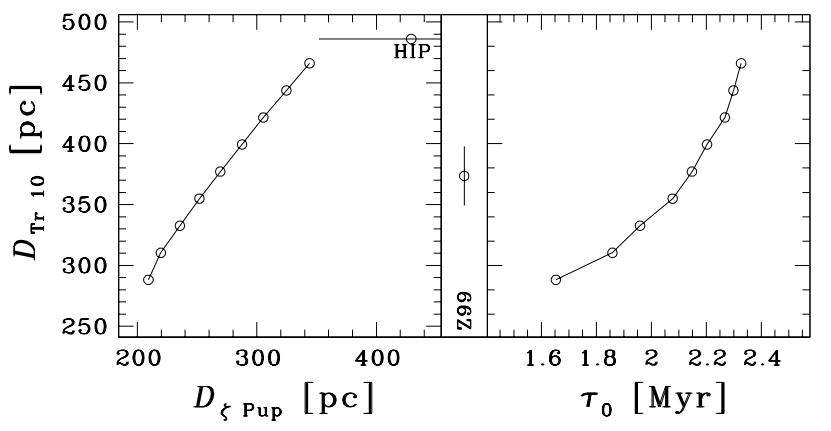

Fig. 14. Predicted distance (left) and kinematic age (right) of $\zeta$ Pup as a function of the distance of Tr 10. The Hipparcos distance and its $1 \sigma$ error are indicated in the left panel. The distance estimate by de Zeeuw et al. (1999, Z99) is indicated in the middle panel

\section{Simulations}

Although we are unable to run our simulations for Vel R2, we conclude that it is not a likely candidate parent association for $\zeta$ Pup. The distance difference between Vel R2 $(\sim 870 \mathrm{pc})$ and $\zeta$ Pup $(\sim 400 \mathrm{pc}$, its canonical distance $)$ is too large. The relative radial velocity between the star and association is $\sim 40 \mathrm{~km} \mathrm{~s}^{-1}\left(v_{\mathrm{rad}, \zeta}\right.$ Pup $=-23.9 \mathrm{~km} \mathrm{~s}^{-1}$ and $\left.v_{\text {rad,Vel R2 }} \sim 20 \mathrm{~km} \mathrm{~s}^{-1}\right)^{8}$. The differences in distance, $\sim 400 \mathrm{pc}$, and velocity, $\sim 40 \mathrm{~km} \mathrm{~s}^{-1}$, between $\zeta$ Pup and Vel R2 yield a kinematic age of $\sim 10 \mathrm{Myr}$. This is older than the expected life time of $\zeta$ Pup (van Rensbergen et al. 1996). Hence Vel R2 is not likely to be the parent group.

Our simulations also show that Vel OB2 is not the parent association. The minimum separation between the association and the runaway star is never smaller than $40 \mathrm{pc}$ for reasonable association distances. Since the association radius is, at maximum, $30 \mathrm{pc}$, we conclude that $\zeta$ Pup has never been inside the boundaries of Vel OB2. We similarly rule out NGC 2391 as parent group.

The simulations for the Trumpler 10 group result in minimum separations of $\geq 10 \mathrm{pc}$. The inferred kinematic age is $\sim 2$ Myr (Fig. 14). Ten parsec is comparable to the radius of $\operatorname{Tr} 10$, so we cannot unambiguously identify or exclude it as the parent association. Furthermore, if $\zeta$ Pup was in or near $\operatorname{Tr} 10$, then its current distance must be 250-350 pc (Fig. 14), which is smaller than the canonical distance of $400 \mathrm{pc}$.

The Vela region contains many young stellar clusters, and suffers from a fair amount of extinction (although $\zeta$ Pup itself is almost unreddened). It is therefore reasonable to assume that we have not yet identified the parent group of $\zeta$ Pup. Similar conclusions were obtained by Vanbeveren et al. (1998), and Vanbeveren, De Loore \& Van Rensbergen (1998).

\footnotetext{
${ }^{8}$ We assume that Vel R2 has a motion similar to all other groups in the direction of the constellation Vela, i.e., $\sim 20 \mathrm{~km} \mathrm{~s}^{-1}$, almost independent of distance.
}

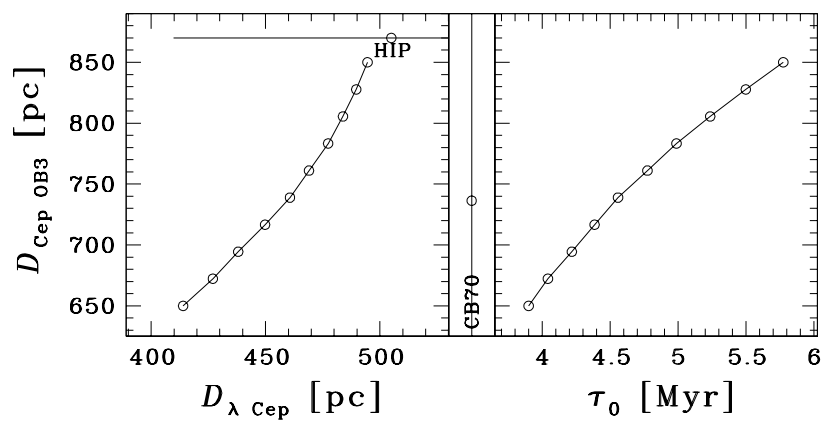

Fig. 15. Predicted distance (left) and kinematic age (right) of $\lambda$ Cep as a function of the distance of Cep OB3. The Hipparcos distance and its $1 \sigma$ error are indicated in the left panel. The distance estimate by Crawford \& Barnes (1970, CB70) is indicated in the middle panel

\section{4. $\lambda$ Cephei, Cepheus OB2 \& Cepheus OB3 (star 22)}

$\lambda$ Cep (HIP 109556) was first classified as a runaway star by Blaauw (Paper I). He noted that its position on the sky, the direction of the proper motion, and the radial velocity were consistent with an origin in the association Cep OB2 (see, e.g., Fig. 22 in de Zeeuw et al. 1999). This luminous supergiant $(\mathrm{O} 6 \mathrm{I}(\mathrm{n}) \mathrm{f})$ is located below Cep OB2 (in Galactic coordinates) and is traveling away from the Galactic plane and Cep OB2. Several other stellar groups are located near Cep OB2, and of these, Cep OB3 may also be a possible parent of $\lambda$ Cep. We consider both associations.

\section{Data}

The data for $\lambda$ Cep are given in Table 3. The phasespace coordinates of Cep OB2 are adopted from de Zeeuw et al. (1999). Unfortunately, the Hipparcos data did not allow these authors to obtain meaningful results for Cep OB3 which is at a distance of $\sim 730$ pc (Crawford $\&$ Barnes 1970). To estimate the phase-space coordinates of Cep OB3 we used the mean position, proper motion, and radial velocity for the Cep OB3 members of Blaauw et al. $(1959):(\ell, b)=\left(110^{\circ} .50,2.91\right) ;\left(\mu_{\ell_{*}}, \mu_{b}\right)=(-2.72 \pm$ $0.28,-1.78 \pm 0.30)$ mas $\mathrm{yr}^{-1} ; v_{\mathrm{rad}}=-17.4 \pm 3.0 \mathrm{~km} \mathrm{~s}^{-1}$.

\section{Simulations}

The orbit calculations show that Cep OB2 cannot be the parent group of $\lambda$ Cep. The simulations do result in small minimum separations between the two, but only for $D_{\lambda \text { Cep }} \sim 250 \mathrm{pc}$ and a kinematic age of $\sim 9$ Myr. These values do not agree with the observed photometric (860 pc, Gies 1987) and trigonometric distances $\left(505_{-95}^{+153} \mathrm{pc}\right.$, Hipparcos) of $\lambda$ Cep, nor with the age of the association (5-7 Myr, de Zeeuw et al. 1999). Moreover, $\lambda$ Cep is a massive $\mathrm{O}$ supergiant, and its lifetime cannot be more than a few million years.

When we run the simulations using Cep OB3 as the parent group we also obtain minimum separations $<10 \mathrm{pc}$. 
Figure 15 shows that the expected distance of the runaway is now $\sim 450 \mathrm{pc}$ and that the kinematic age is $\sim 4.5 \mathrm{Myr}$. This is a little on the large side for the nominal lifetime of a $40 M_{\odot}$ star, but might not be impossible. Cep OB3 consists of two subgroups with ages of 5.5 (subgroup $b$ ) and 7.5 Myr (subgroup a) (e.g., Jordi et al. 1996). Considering the high helium abundance and large rotational velocity of $\lambda$ Cep, subgroup $a$ is a likelier parent of the runaway than subgroup $b$, since the age difference between the subgroup and the runaway is $3 \mathrm{Myr}$ for $a$. For subgroup $b$ this is only $1 \mathrm{Myr}$, leaving little time for binary evolution. We conclude that $\lambda$ Cep is likely to have become a runaway star as the result of a supernova explosion in a binary system in subgroup $a$ of Cep OB3 $\sim 4.5$ Myr ago.

\section{Identification of new parent groups}

The orbit retracing technique allows us to identify the (likely) parent group for thirteen "new" single runaways, one new pair, two more pulsars, and Geminga from the samples defined in Sect. 2. Little is known about most of these objects, so our discussion is relatively brief. The results are summarized in Table 5 .

\subsection{HIP 3881 \& Lacerta OB1}

(star 1)

The orbit of the spectroscopic binary $\nu$ Andromedae $(\mathrm{B} 5 \mathrm{~V}+\mathrm{F} 8 \mathrm{~V})$ intersected the Lac OB1 association 8-10 Myr ago. If this is the parent group, then the kinematic age is comparable to the (uncertain) age of the association $(\sim 10 \mathrm{Myr})$. This, together with a normal rotational velocity for the primary $\left(80 \mathrm{~km} \mathrm{~s}^{-1}\right)$ and the binary nature (two main-sequence stars) of this runaway, suggests that it was formed by dynamical ejection.

\subsection{HIP 22061, HIP 29678, Geminga \& the $\lambda$ Ori region (stars 4 and 7 and neutron star 9)}

The two stars form an analog of the runaway pair AE Aur and $\mu \mathrm{Col}$ (Sect. 4). They also move in opposite directions and with similar space motions: $86.5 \mathrm{~km} \mathrm{~s}^{-1}$ for HIP 22061 (B2.5V) and $63.0 \mathrm{~km} \mathrm{~s}^{-1}$ for HIP $29678^{9}$ (B1V) (velocities are with respect to their own standard of rest). Retracing the orbits of HIP 22061 and HIP 29678, we find that the two stars were close together $\sim 1.1 \mathrm{Myr}$ ago, suggesting that these two runaways were also formed by the DES. We did not find a possible third party (i.e., another runaway in the neighbourhood or a massive binary).

Applying the principle of conservation of linear momentum at the time of the encounter, as we did in Sect. 4, we can predict the properties of the parent cluster. We can only use these two stars and not three as in Sect. 4.5. We find that the parent cluster should be located around $(\ell, b) \sim(196.5,-12.0)$. This coincides with the $\lambda$ Orionis star-forming region (e.g., Gomez \& Lada 1998;

\footnotetext{
${ }^{9}$ HIP 29678 is a visual double star. Gies \& Bolton (1986) did not detect any radial velocity variations.
}

Dolan \& Mathieu 1999), which contains at least three young stellar clusters (the $\lambda$ Ori cluster and the clusters associated with the dark clouds B30 and B35) and is surrounded by the $\lambda$ Orionis ring. Several authors have suggested that this expanding ring of molecular clouds is the result of a supernova explosion $\sim 0.35 \mathrm{Myr}$ ago (e.g., Cunha \& Smith 1996). The predicted cluster position does not coincide with one of the three star clusters. Furthermore, the predicted radial velocity of the cluster, $\sim 10 \mathrm{~km} \mathrm{~s}^{-1}$, differs significantly from that of the $\lambda$ Ori clusters, $\sim 24 \mathrm{~km} \mathrm{~s}^{-1}$. However, these differences might be erased if a third body (either a single star or a binary) was involved (cf. Sect. 4) ${ }^{10}$.

The conclusion that the DES is the acting mechanism for these runaways is supported by (i) the youth of the clusters in the $\lambda$ Ori star-forming region, 2-6 Myr (Dolan \& Mathieu 1999), (ii) the density of these clusters (Dolan \& Mathieu), and (iii) the small rotational velocity of HIP 29678, <25 $\mathrm{km} \mathrm{s}^{-1}$ (Morse et al. 1991).

It is worth mentioning that HIP 22061 and HIP 29678 are not the only objects running away from the $\lambda$ Orionis region. Frisch (1993) and Smith et al. (1994) suggested that the neutron star Geminga also originated from this star-forming region (Fig. 2; but see Bignami \& Caraveo 1996). Moreover, the age of Geminga ( $350000 \mathrm{yr}$ ) agrees well with the time of the supernova explosion which created the $\lambda$ Orionis ring.

\subsection{HIP 38455 \& Collinder 135}

(star 8)

The ellipsoidal variable star HIP 38455 (B2V) moves away from the open cluster Collinder 135 with a velocity of almost $40 \mathrm{~km} \mathrm{~s}^{-1}$, mostly in the radial direction. The orbits of the runaway and cluster intersected each other about $3 \mathrm{Myr}$ ago; this is significantly smaller than the age of the open cluster: $\sim 35$ Myr. This difference, and the large rotational velocity $\left(v_{\text {rot }} \sin i=212 \mathrm{~km} \mathrm{~s}^{-1}\right)$, suggest that the runaway originated via the BSS. Ellipsoidal variable stars show brightness variation because of their non-spherical shapes. The deformation of the star is thought to be due to tidal interactions with a companion star (Beech 1985). The fact that HIP 38455 is double (cf. Häfner \& Drechsel 1986) suggests that the kick velocity of the compact object created in the supernova explosion was small and that the binary remained bound. This system might in the future become a high-mass X-ray binary (Sect. 1).

\subsection{HIP 38518 \& Vela OB2}

(star 9)

This B0.5Iab supergiant is located behind the Vel OB2 association and is currently moving away from it. Retracing

\footnotetext{
10 Dolan \& Mathieu (1999) report that long term monitoring of $\lambda$ Ori's radial velocity indicates that this star is a binary with a systematic velocity $\left(v_{\text {rad }} \sim 18 \mathrm{~km} \mathrm{~s}^{-1}\right)$ which differs by $6-8 \mathrm{~km} \mathrm{~s}^{-1}$ from that of the clusters in the $\lambda$ Ori region. However, the simulations show that $\lambda$ Ori cannot be related to the event that created the runaways HIP 22061 and HIP 29678.
} 
the orbit shows that $\sim 6$ Myr ago the star was located inside the Vel OB2 association. This kinematic age is smaller than the association age (10-15 Myr). Adding to this the large rotational velocity $\left(v_{\text {rot }} \sin i=220 \mathrm{~km} \mathrm{~s}^{-1}\right)$ and the blue straggler character of HIP 38518 (see Sect. 9 for a further discussion), we infer that the BSS is the preferred scenario.

\subsection{HIP 42038 \& Upper Centaurus Lupus or IC 2391}

(star 11)

The path of the B3V runaway star HIP 42038 traversed the open cluster IC $2391 \sim 6 \mathrm{Myr}$ ago and the Upper Centaurus Lupus association $\sim 8 \mathrm{Myr}$ ago. Little is known about HIP 42038, making it difficult to determine its origin. The only available criterion is that the kinematic age is smaller than both the age of IC 2391 (45 Myr) and the age of Upper Centaurus Lupus (13 Myr) favoring the BSS.

\subsection{HIP 46950 \& IC 2602}

(star 12)

Somewhere between $2 \mathrm{Myr}$ and $10 \mathrm{Myr}$ ago the B1.5IV star occupied the same space as the open cluster IC 2602. The only bit of information available for the identification of the runaway's origin is the difference between the kinematic age and the age of the open cluster ( $\sim 25 \mathrm{Myr})$. This would suggest that the BSS is the origin.

\subsection{HIP 48943 \& Lower Centaurus Crux}

(star 13)

HIP 48943 is a runaway from the Lower Centaurus Crux association. The orbit of the B5Ve star intersected the association center $\sim 4 \mathrm{Myr}$ ago. The runaway has a rotational velocity of $230 \mathrm{~km} \mathrm{~s}^{-1}$ and its kinematic age is significantly smaller than the age of Lower Centaurus Crux, 10 Myr. The BSS is thus the most likely formation mechanism.

\subsection{HIP 49934 \& IC 2391 or IC 2602}

(star 14)

The retraced orbit of the emission-line star HIP 49934 (B2IVnpe) intersects two open clusters: IC $2391 \sim 3 \mathrm{Myr}$ ago and IC $2602 \sim 6$ Myr ago. The large difference between the nuclear ages of both clusters (45 Myr and $25 \mathrm{Myr}$, respectively) and the kinematic age indicates that the BSS is the most likely origin of HIP 49934. This assumption is supported by the large rotational velocity $\left(v_{\text {rot }} \sin i=\right.$ $280 \mathrm{~km} \mathrm{~s}^{-1}$ ).

\subsection{HIP 57669 \& IC 2602}

(star 15)

About 3 Myr ago the emission-line star HIP 57669 left the open cluster IC 2602. This B3Ve star has a large rotational velocity $\left(v_{\mathrm{rot}} \sin i=251 \mathrm{~km} \mathrm{~s}^{-1}\right)$ and its kinematic age differs significantly from the age of IC 2602. Both these points suggest the BSS as the origin of HIP 57669.

\subsection{HIP 69491 \& Upper Centaurus Lupus or Cepheus OB6}

(star 16)

This candidate runaway is an eclipsing binary (B5V). Its path traverses the Upper Centaurus Lupus association and the Cep OB6 association. The respective kinematic ages are 3 and 10 Myr. Both these kinematic ages are smaller than the association ages of $13 \mathrm{Myr}$ for Upper Centaurus Lupus and $\sim 50$ Myr for Cep OB6. This discrepancy in ages combined with the binary nature of the candidate runaway excludes both the standard BSS and the DES and might suggest that neither Upper Centaurus Lupus nor Cep OB6 is the parent of HIP 69491. The BSS is highly unlikely because the eclipses imply two objects of similar size and not, for example, a main-sequence star and a compact object. The DES is excluded because both associations were no longer compact/dense enough for dynamical encounters to be efficient at the time of ejection.

What other mechanisms do exist to create a fast moving $\left(v_{\text {space }}=77 \mathrm{~km} \mathrm{~s}^{-1}\right)$ binary system? One possibility is a supernova explosion in a triple system consisting of a hard binary and a third star with a larger semi-major axis (i.e., a stable triple system). This would result in either (i) a hard binary moving at moderate speed $\left(<30 \mathrm{~km} \mathrm{~s}^{-1}\right)$ or (ii) a fast runaway and a normal field star. In the latter case one of the stars in the binary explodes and creates a fast runaway. The third star, being weakly bound to the system would hardly be affected by the explosion. In the former case the single star explodes causing the binary to start moving at the orbital speed it had within the triple system. This velocity should be small since the binary is much more massive than the third star. However, neither case would create a runaway binary-system like HIP 69491. Whereas it is likely that the star originated in either Upper Centaurus Lupus or Cep OB6, the mechanism that formed this runaway remains unknown.

\subsection{HIP 76013 \& Lower Centaurus Crux}

$(\operatorname{star} 17)$

$\kappa^{1}$ Apodis is a B1npe emission-line star, and is the brightest component of a visual double system. This star has a radial velocity of $62 \mathrm{~km} \mathrm{~s}^{-1}$ and is moving away from the Galactic plane. Its orbit intersects the Lower Centaurus Crux subgroup of Sco OB2 2-3 Myr ago. Because of the large difference in kinematic age and association age ( $\sim 10 \mathrm{Myr})$ the BSS is the most likely explanation for the runaway nature of $\kappa^{1}$ Aps.

\subsection{HIP 82868 \& IC 2602}

(star 19)

Little is known about the B3Vnpe star HIP 82868 whose orbit intersects that of the IC 2602 open cluster some 6 Myr ago. A firm identification of the formation mechanism is difficult, since we only know that the kinematic age differs from the age of IC 2602 (25 Myr). This suggests that HIP 82686 is a BSS runaway. 


\subsection{HIP 91599 \& Perseus OB2 or Perseus OB3}

(star 20)

HIP 91599 is a known runaway star (B0.5V; Vitrichenko et al. 1965); however, its parent association/cluster has never been identified. The simulations show that HIP 91599 originates from Per OB2 or Per OB3. The two associations have very different ages $(\sim 7 \mathrm{Myr}$ for Per OB2 and $\sim 50$ Myr for Per OB3). The kinematic age of HIP 91599 is $\sim 8 \mathrm{Myr}$ and $\sim 6 \mathrm{Myr}$ for Per OB2 and Per OB3, respectively. Since we lack information on the rotational velocity and the helium abundance, we are unable to conclude whether HIP 91599 is a DES runaway from Per OB2 or a BSS runaway from Per OB3.

\subsection{HIP 102274 \& Cepheus OB2}

(star 21)

The B5 star HIP 102274 was located at the center of the Cep OB2 association between two and three Myr ago, when the association was 3-4 Myr old. This kinematic age coincides with the time of the supernova explosion proposed by Kun et al. (1987) to explain the characteristics of the Cepheus bubble, a ring-like structure of infrared emission. Taken together, this is strong circumstantial evidence for HIP 102274 being a BSS runaway.

\subsection{PSR J0826+2637, PSR J1115+5030 \& Perseus OB3 \\ (pulsars 1 and 4)}

The orbits of the pulsars J0826+2637 and J1115+5030 intersect that of the Per OB3 association. This group contains the $\alpha$ Persei open cluster, and is often referred to as the Cassiopeia-Taurus association (de Zeeuw et al. 1999). The simulations show that if Per OB3 is the parent of PSR J0826+2637, its kinematic age is $\sim 1 \mathrm{Myr}$ and its radial velocity is $\sim 100 \mathrm{~km} \mathrm{~s}^{-1}$. For PSR J1115+5030 we predict a kinematic age of $\sim 1.5 \mathrm{Myr}$ and a radial velocity of $\sim 150 \mathrm{~km} \mathrm{~s}^{-1}$. The characteristic ages $(P /(2 \dot{P}))$ of the pulsars are 4.9 Myr and $10 \mathrm{Myr}$ for PSR J0826+2637 and PSR J1115+5030, respectively. The unknown radial velocity of these pulsars makes it difficult to prove beyond doubt that these pulsars were born in the Per OB3 association. Although Fig. 2 shows that the orbits projected on the sky do not differ much for different radial velocities, and that they cross Per OB3, they may also cross the paths of other, more distant, associations or clusters not shown in the figure.

If the two pulsars orginated in Per OB3, then the initial periods would be $0.47 \mathrm{~s}$ for $\mathrm{J} 0826+2637$ and $1.53 \mathrm{~s}$ for J1115+5030, assuming no glitches occurred. The latter value is large, which might indicate that this pulsar travelled longer, from another site of origin.

It is not unlikely to find many pulsars associated with Per OB3 since its age, $\sim 50 \mathrm{Myr}$, is comparable to the main-sequence life-time of an $8 M_{\odot}$ star. These are the least massive stars to explode as a supernova. Since the moment at which a star explodes, $\tau_{\mathrm{SN}}$, depends on its mass, $\left(\tau_{\mathrm{SN}} \sim M^{-\alpha}\right.$, where $\alpha>0$ and $\left.M>8 M_{\odot}\right)$ and the number of stars of mass $M, N(M)$, also depends on the mass $\left(N(M) \sim M^{-\beta}\right.$, where $\left.\beta>0\right)$, the number of supernovae increases with time $\left(N_{\mathrm{SM}} \sim \tau^{\beta / \alpha}\right.$, for $M>$ $\left.8 M_{\odot}\right)$. The number of supernovae, and thus the number of pulsars, will thus increase with time until the stars of $8 M_{\odot}$ have exploded as supernovae. Afterwards the pulsar production rate will drop to almost zero.

\subsection{The Vela pulsar}

(pulsar 2)

PSR J0835-4510 is only 10000 yr old, and therefore has not travelled far from its birth place $\left(\sim 9^{\prime}\right.$ on the sky), the Vela star-forming region at $\sim 450$ pc. It lies within the boundaries of the $\sim 10$ Myr old Vel OB2 association (de Zeeuw et al. 1999), which is the likely parent group.

\section{Runaways and pulsars without parents?}

The sample of runaways and pulsars we have analysed here is severely incomplete (see Sect. 2.1). Furthermore, we have only a limited knowledge of where massive stars form in the Solar neighbourhood. The case of $\zeta$ Pup described in Sect. 5 shows that some local parent groups may not yet have been found. Beyond $\sim 500 \mathrm{pc}$, only the large complexes such as Cygnus OB1/OB2 or Sco OB1 are well documented. The accuracy of the three-dimensional positions and velocities of these distant star-forming regions is poor, making determination of reliable orbits very difficult. Runaway stars of spectral types B0 and later are especially difficult to link to a parent group. These stars have main-sequence lifetimes of up to several tens of Myr and can therefore travel far (several kpc) from their places of origin. A similar argument holds for the pulsars. We suspect that most of the runaway stars and pulsars in our sample for which we were not able to identify a parent group originated outside the Solar neigbourhood.

An example is the B2.5V star $72 \mathrm{Col}$, HIP 28756 (van Albada 1961; the asterisk at $[\ell, b] \sim\left[238^{\circ},-23^{\circ}\right]$ in Fig. 2). It has a peculiar velocity of $\sim 200 \mathrm{~km} \mathrm{~s}^{-1}$ and its parent association is Sco OB1, at a distance of $\sim 2 \mathrm{kpc}$ (Humphreys 1978). Van Albada derived a kinematic age of 14 Myr for $72 \mathrm{Col}$, based on a simple model of Galactic rotation (Kwee et al. 1954). This star does not appear in Sects. 5 and 6 because its path did not carry it through one of the nearby associations. The Solar neighbourhood thus not only contains runaways for which the parent associations are also nearby, but it also contains runaways which originated far from the Sun.

Another star that immediately catches the eye in Fig. 2 is HIP 94899 (the asterisk at $[\ell, b] \sim\left[341^{\circ},-26^{\circ}\right]$ ). This double star of spectral type B3Vn has a radial velocity of $151 \mathrm{~km} \mathrm{~s}^{-1}$, and its path seems to cross the Per OB3 association. However, our simulations show that the runaway never comes within $40 \mathrm{pc}$ of the association, implying that this system must have another, unknown, parent.

We have seen in Sect. 3 that PSR J1239+2453 most likely originates outside the Solar neighbourhood. We did not find a parent group for the remaining pulsars because 

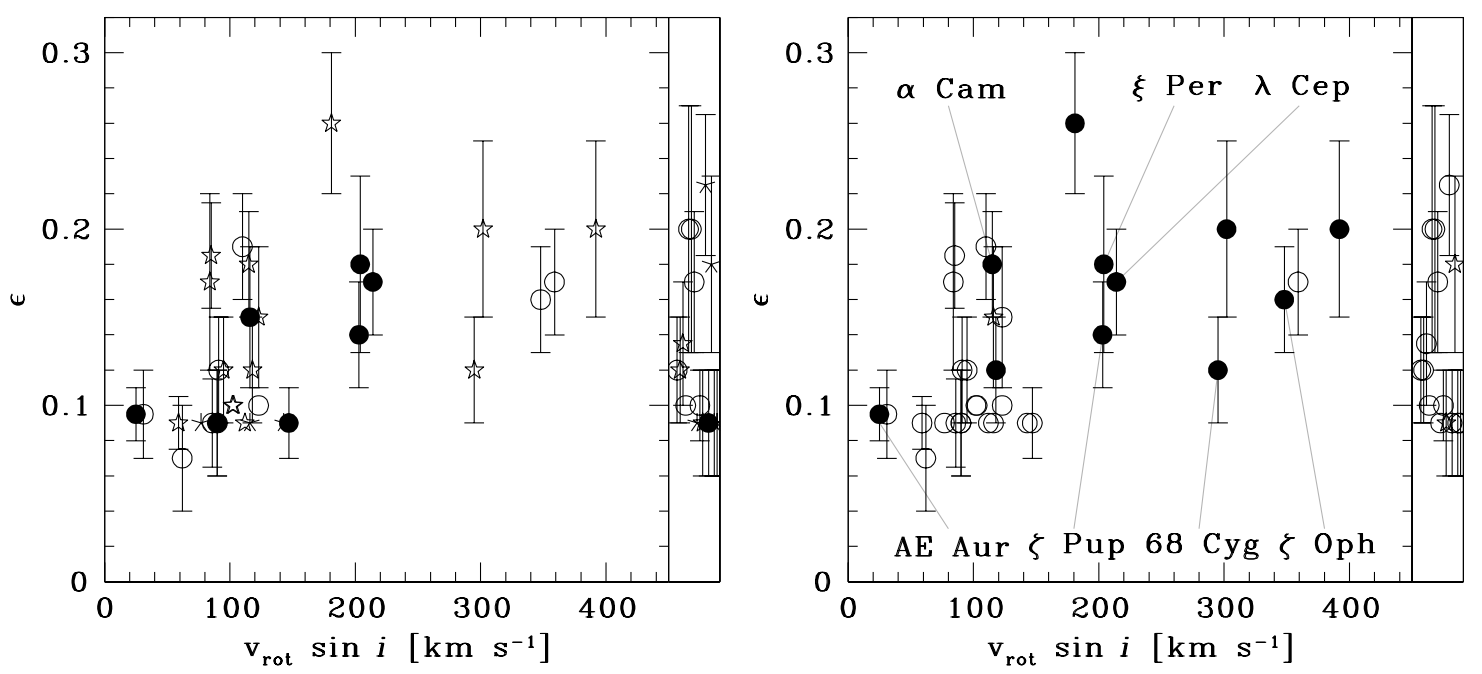

Fig. 16. Helium abundance ( $\epsilon$ : \# relative to Hydrogen) versus rotational velocity for O stars. Left: open circles: stars with $|v| \leq 30 \mathrm{~km} \mathrm{~s}^{-1}$, filled circles: stars with $|v|>30 \mathrm{~km} \mathrm{~s}^{-1}$, stars: stars with $\pi-\sigma_{\pi}<0$ mas, and asterisks: stars without Hipparcos data. Right: open circles: non-runaway stars, filled circles: runaway stars, and stars: doubtful runaways. The right of each panel display the helium abundances of stars with an unknown rotational velocity. See text for details

(i) an unreasonably large radial velocity $\left(>500 \mathrm{~km} \mathrm{~s}^{-1}\right)$ is necessary for the paths of the pulsar and parent group to intersect (PSR J1135+1551), just as we found for PSR J1239+2453, or (ii) the past orbit simply does not intersect any of the nearby young stellar groups (PSR J0953+0755 and PSR J1456-6845).

\section{Helium abundance versus rotational velocity}

Blaauw (1993) pointed out that most of the reliable runaways known at the time have high helium abundances $(\epsilon \gtrsim 0.13)$ and large rotational velocities $\left(v_{\text {rot }} \sin i \gtrsim\right.$ $150 \mathrm{~km} \mathrm{~s}^{-1}$ ), whereas most of the non-runaways have normal helium abundances and rotational velocities. Only one classical runaway star, AE Aur, does not follow this trend, and this was already suspected to be the result of the DES rather than the BSS. Blaauw suggested that the $\left(\epsilon, v_{\text {rot }} \sin i\right)$-diagram of the small sample of known runaway stars supported the conjecture that most runaways are produced by the BSS, since these characteristics are natural consequences of close binary evolution (Sect. 1; van den Heuvel 1985).

As shown in Table 3, only five of the 23 runaways listed there have a measurement of $\epsilon$, and six do not have a measured rotational velocity. In order to pursue Blaauw's suggestion, we therefore constructed a sample of O stars with known rotational velocities (Penny 1996) and helium abundances (Kudritzki \& Hummer 1990; Herrero et al. 1992). We also determined, based on Hipparcos astrometry and Hipparcos Input Catalogue radial velocities, the space velocities of these stars with respect to their local standard of rest. The $\left(\epsilon, v_{\text {rot }} \sin i\right)$ diagram in the left panel of Fig. 16 shows that these $\mathrm{O}$ stars can roughly be divided into three groups: (i) those with small rotational velocities, $v_{\text {rot }} \sin i \lesssim 80 \mathrm{~km} \mathrm{~s}^{-1}$, and normal helium abundances, $\epsilon \sim 0.09$, (ii) those with moderate rotational velocities, $80 \lesssim v_{\text {rot }} \sin i \lesssim 150 \mathrm{~km} \mathrm{~s}^{-1}$, and normal to high helium abundances, $0.09 \lesssim \epsilon \lesssim 0.2$, and (iii) those with large rotational velocities, $v_{\text {rot }} \sin i \gtrsim 150 \mathrm{~km} \mathrm{~s}^{-1}$, and high helium abundances, $\epsilon \gtrsim 0.14$. The symbols in the left panel of Fig. 16 are chosen according to the magnitude of the space velocity. The stars represented by filled circles have space velocities $v_{\text {space }} \geq 30 \mathrm{~km} \mathrm{~s}^{-1}$, and the open circles have $v_{\text {space }}<30 \mathrm{~km} \mathrm{~s}^{-1}$; the other symbols indicate stars for which no Hipparcos data are available (asterisks) or for stars with insignificant Hipparcos data (starred).

The left panel of Fig. 16 does not show an obvious separation between runaways (filled circles) and non-runaways (open circles). However, based on the available data for each star (position, radial velocity, distance modulus, cluster membership) it is possible to decide whether the star is a runaway. The result is shown in the right panel of Fig. 16; it turns out that many of the stars with insignificant Hipparcos data are runaways (including some classical runaways), and that some of the stars indicated as highvelocity stars in the left panel are members of a cluster which has a peculiar motion. The resulting $\left(\epsilon, v_{\text {rot }} \sin i\right)$ diagram now shows a clear separation between the runaway stars and the normal O stars. Except for AE Aur (Sect. 4) all runaways have high helium abundances, $\epsilon \gtrsim 0.12$, and large rotational velocities, $v_{\text {rot }} \sin i \gtrsim 150 \mathrm{~km} \mathrm{~s}^{-1}$. Only one star in this area of the $\left(\epsilon, v_{\text {rot }} \sin i\right)$ diagram is not indicated as a runaway: the double star HIP 113306 (OV7n). This star is a member of Cep OB3 $(\epsilon=0.17$, $v_{\text {rot }} \sin i \sim 359 \mathrm{~km} \mathrm{~s}^{-1}$ ).

We thus confirm Blaauw's conclusion that massive runaways predominantly have high helium abundances and large rotational velocities, suggesting that they are formed mainly by the binary-supernova scenario. However, this conclusion is based on a limited sample which is by no 


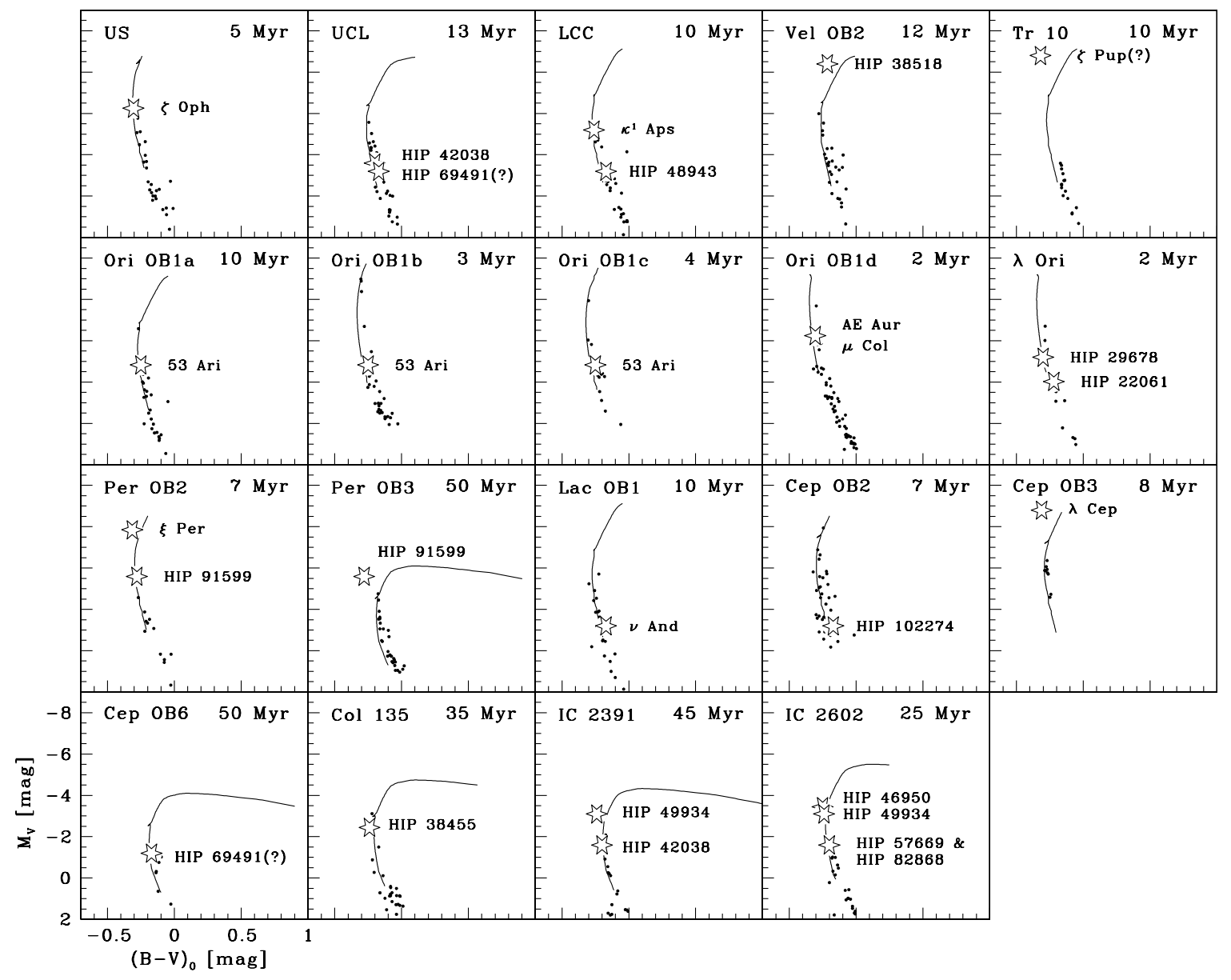

Fig. 17. Colour vs. absolute magnitude diagrams of the runaways (stars) and their parent association/cluster (small dots). The association/cluster members have been de-reddened using the Q-method. The colours and absolute magnitudes of the runaways have been determined using their spectral types (Table 3; Schmidt-Kaler 1983). The isochrones are from Schaller et al. (1992) for Solar metallicity and standard mass loss. The ages of the associations are indicated in the top right of each panel (US: Upper Scorpius, UCL: Upper Centaurus Lupus, LCC: Lower Centaurus Crux)

means statistically complete. A systematic survey of the radial velocities, rotational velocities and chemical abundances of the early-type stars in the Solar neighbourhood is highly desirable.

\section{Blue stragglers}

One of the characteristics of runaway stars produced according to the binary-supernova scenario is that these stars are expected to be blue stragglers (Sect. 1). The mass transfer in close binary systems from the primary to the secondary prior to the supernova explosion deposits a large amount of hydrogen onto the future runaway. This new supply of fuel makes the runaway appear younger than the association/cluster in which it was born, i.e., it appears rejuvenated.

Figure 17 shows the colour vs. absolute magnitude diagrams of the parent clusters discussed in this paper. The association members (dots) have been de-reddened following the Q-method; only the early-type members (A0 and earlier) are shown. The solid lines denote the Schaller et al. (1992) isochrones for Solar metallicity and a standard mass loss rate for the ages of the associations. The runaway stars are denoted by starred symbols. A runaway can appear in more than one panel if two or more possible parents have been identified. The $B-V$ colour and absolute magnitude have been determined using the spectral type of the runaways (Table 3; Schmidt-Kaler 1982). Three stars in Fig. 17 clearly are blue stragglers: HIP $38518, \xi$ Per, and $\lambda$ Cep; and three others could be blue stragglers depending on the correct identification of the parent: $\zeta$ Pup, HIP 49934, and HIP 91599. The latter three stars have uncertain parent identifications (see Sects. 5 and 6 ). The blue straggler nature of the former three stars confirms their identification as BSS runaway (see Table 5). The star $\zeta$ Oph has also been claimed to be a blue straggler (e.g., Blaauw 1993). However, the Upper Scorpius panel shows that $\zeta \mathrm{Oph}$ is the bluest star of the group, but it lies on the main sequence, as also found by de Geus et al. (1989) on the basis of uvby $\beta$ photometry. 
By contrast to the BSS runaways, those produced by the DES are expected to follow the main sequence of the parent group. These runaways most likely did not experience a period of binary evolution in which mass transfer was important. The runaway stars which we identified securely as DES runaways (AE Aur, $\mu$ Col, HIP 22061, and HIP 29678) indeed fall on the main sequence of their parents (the Trapezium and the $\lambda$ Ori cluster).

\section{Concluding remarks}

We have used the Hipparcos astrometry for a sample of nearby candidate OB runaway stars to locate their parent groups, and to identify their formation mechanisms. We retraced the orbits of these candidate runaways, and determined where and when they passed through a possible parent group. We find that both mechanisms proposed for the production of runaway stars, the binary-supernova scenario (BSS) and the dynamical ejection scenario (DES), operate. Table 5 summarizes the results. Even though the number of runaways discussed in this paper is small, and the weight of evidence varies, we find that roughly $2 / 3$ of the runaways is produced by the BSS and $1 / 3$ by the DES. This agrees with the results of binary population synthesis calculations by Portegies Zwart (2000). At present it is not feasible to extend this study to other runaways. This is mainly due to large uncertainties in the velocities and distances of the runaways, and the limited knowledge of star-forming regions with distances $>500 \mathrm{pc}$.

Tracing the runaway orbits back in time provides, for the first time, direct evidence that both scenarios produce single runaway stars (Hoogerwerf et al. 2000). The orbit calculations demonstrate that the runaway $\zeta \mathrm{Oph}$ and the progenitor of PSR J1932+1059 once formed a binary system in the Upper Scorpius association, and that the neutron star acquired a kick velocity of $\sim 350 \mathrm{~km} \mathrm{~s}^{-1}$ in the supernova explosion. The runaways AE Aur and $\mu \mathrm{Col}$, and the binary $\iota$ Ori were involved in a dynamical interaction (a binary-binary collision) $\sim 2.5 \mathrm{Myr}$ ago, which took place in the Trapezium cluster.

The current investigation is biased towards finding BSS runaways. This is mainly due to the fact that the accuracy of the available data, and our knowledge of the location and motions of star-forming regions, restrict the study to $\sim 700 \mathrm{pc}$. The small volume implies that we are only able to identify runaway stars with small kinematic ages of 0-10 Myr (i.e., runaways which recently left their parent association). Runaways which were created at an earlier time have most likely traveled outside our sample limits. Since associations and open clusters can create BSS runaways during $\sim 50 \mathrm{Myr}$ (approximately the lifetime of a $8 M_{\odot}$ star) and DES runaways only in the inital stages when the group still has a high density, we expect to find more BSS than DES runaways because there are relatively many more old parents than young parents in the Solar neighbourhood. This bias is somewhat weakened by the fact that most dynamical interactions produce
Table 5. Parent associations, kinematic ages $\left(\tau_{0}\right)$ and formation mechanisms of the runaways and neutron stars discussed in this paper. For runaway stars which have more than one possible parent we give the results for the individual parent associations/clusters. The last column indicates the number of the runaway/pulsar in Fig. 2. Abbreviations: US: Upper Scorpius; UCL: Upper Centaurus Lupus; LCC: Lower Centaurus Crux; BSS: binary supernova scenario; DES: dynamical ejection scenario

\begin{tabular}{|c|c|c|c|c|c|}
\hline HIP & Name & Parent & $\begin{array}{c}\tau_{0} \\
{[\mathrm{Myr}]}\end{array}$ & Origin & Fig. 2 \\
\hline 3881 & $\nu$ And & Lacerta OB1 $b$ & 9.0 & DES & 1 \\
\hline \multirow[t]{3}{*}{14514} & 53 Ari & Orion OB1 a & 4.3 & BSS & 2 \\
\hline & & Orion OB1 $b$ & 4.8 & DES & 2 \\
\hline & & Orion OB1 $c$ & 5.0 & DES & 2 \\
\hline 18614 & $\xi$ Per & Perseus OB2 & 1.0 & BSS & 3 \\
\hline 22061 & & $\lambda$ Ori SFR & 1.1 & DES & 4 \\
\hline 24575 & AE Aur & Trapezium & 2.5 & DES & 5 \\
\hline 27204 & $\mu \mathrm{Col}$ & Trapezium & 2.5 & DES & 6 \\
\hline 29678 & & $\lambda$ Ori SFR & 1.1 & DES & 7 \\
\hline 38455 & & Collinder 135 & 3.0 & BSS & 8 \\
\hline 38518 & & Vela OB2 & 6.0 & BSS & 9 \\
\hline 39429 & $\zeta \mathrm{Pup}$ & ? & & & 10 \\
\hline \multirow{2}{*}{42038} & & UCL & 8.0 & BSS & 11 \\
\hline & & IC 2391 & 6.0 & BSS & 11 \\
\hline 46950 & & IC 2602 & $2-10$ & BSS & 12 \\
\hline 48943 & & $\mathrm{LCC}$ & 4.0 & BSS & 13 \\
\hline \multirow[t]{2}{*}{49934} & & IC 2391 & 3.0 & BSS & 14 \\
\hline & & IC 2602 & 6.0 & BSS & 14 \\
\hline 57669 & & IC 2602 & 3.0 & BSS & 15 \\
\hline \multirow[t]{2}{*}{69491} & & $\mathrm{UCL}(?)$ & 3.0 & ? & 16 \\
\hline & & Cepheus OB6(?) & 10.0 & ? & 16 \\
\hline 76013 & $\kappa^{1}$ Aps & $\mathrm{LCC}$ & 2.5 & BSS & 17 \\
\hline 81377 & $\zeta \mathrm{Oph}$ & US & 1.0 & BSS & 18 \\
\hline 82868 & & IC 2602 & 6.0 & BSS & 19 \\
\hline \multirow[t]{2}{*}{91599} & & Perseus OB2 & 8.0 & DES & 20 \\
\hline & & Perseus OB3 & 6.0 & BSS & 20 \\
\hline 102274 & & Cepheus OB2 & 2.5 & BSS & 21 \\
\hline \multirow[t]{6}{*}{109556} & $\lambda$ Cep & Cepheus OB3 & 4.5 & BSS & 22 \\
\hline & J0826+2637 & Perseus OB3 & 1.0 & ? & 1 \\
\hline & J0835-4510 & Vela OB2 & 0.01 & ? & 2 \\
\hline & $\mathrm{J} 1115+5030$ & Perseus OB3 & 1.5 & ? & 4 \\
\hline & $\mathrm{J} 1932+1059$ & US & 1.0 & BSS & 8 \\
\hline & Geminga & $\lambda$ Orionis & 0.35 & ? & 9 \\
\hline
\end{tabular}

two runaway stars while the binary-supernova mechanism produces only one.

The creation of runaway stars modifies the mass function of the parent group at the high-mass end, where the total number of stars is small. For example, the encounter in Orion described in Sect. 4 removed four stars with a total mass of order $70 M_{\odot}$ from the Trapezium cluster, while only six stars more massive than $10 M_{\odot}$ remain. Derivation of the initial mass function of young stellar groups from the present-day mass function without accounting for the associated runaway stars leads to erroneous results.

Our Hipparcos-based study has identified 56 runaway stars within $700 \mathrm{pc}$ from the Sun, and tripled the subset of these for which a parent group is known (from 6 to 21). As mentioned in Sect. 2, less than a third of the O-B5 stars in the Hipparcos Catalog have a measured radial velocity. Obtaining these is likely to result in another factor of three increase in the size of the sample, so that statistical studies become possible.

The next major step in our understanding of the origin of runaway stars will come when large datasets of microarcsecond ( $\mu$ as) astrometry and accurate radial velocities (1-2 $\mathrm{km} \mathrm{s}^{-1}$ ) become available. Distances accurate to a few parsec will allow for a final confirmation or rejection 
of the genetic link between runaways and their parents (e.g., Fig. 12). These data will become available over the next two decades with the launches of several astrometric satellites (FAME, SIM, GAIA). These aim to obtain $\mu$ as astrometry for a large number of stars, from 10000 stars with SIM to 1 billion stars with GAIA. Besides astrometry, accurate radial velocities are also required; unfortunately, there is no dedicated effort to obtain these for a large number of $\mathrm{O}$ and $\mathrm{B}$ stars.

The BSS and DES can produce runaway stars with spectral types beyond B5 (e.g., Kroupa 2000b; Portegies Zwart 2000). These will be harder to find, as the velocity distribution of the later-type stars in the Galactic disk is broader than for the O-B5 stars, and the fractional production of low-mass runaways is small. Identifying their parent groups is also harder, because these stars may have traveled for much longer times. However, $\mu$ as accuracy astrometry complemented with accurate radial velocities will undoubtedly reveal such objects, and will provide further constraints on the binary fraction and the binary mass-ratios in open clusters and associations.

Figure $1 \mathrm{~b}$ shows that there are 19 additional pulsars within one kpc for which an accurate proper motion is not available. A systematic program to measure these might allow the detection of more examples of pairs such as $\zeta$ Oph and PSR J1932+1059. It would also improve the characterisation of the pulsar population as a whole. VLBI techniques hold the promise of achieving sub-mas astrometry (positions, proper motions, and parallaxes) in the near future.

Acknowledgements. It is a pleasure to thank Bob Campbell for a discussion on VLBI proper motions of pulsars, Rob den Hollander for writing an early version of the software used here, Nicolas Cretton for providing the Galactic potential used in the orbit integrations, and Ed van den Heuvel, Lex Kaper, Michael Perryman, the referee Walter van Rensbergen, and in particular Adriaan Blaauw, for stimulating comments and suggestions. This research was supported by the Netherlands Foundation for Research in Astronomy (NFRA) with financial aid from the Netherlands Organization for Scientific Research (NWO).

\section{Appendix A: Distribution of differences}

In this appendix we record the distribution of the difference between two observables, each of which has a Gaussian error distribution. We define two observable quantities, $x_{1}$ and $x_{2}$, which are both distributed normally, $G_{1}\left(x_{1} ; \mu_{1}, \sigma_{1}\right)$ and $G_{2}\left(x_{2} ; \mu_{2}, \sigma_{2}\right)$, respectively, where $\mu_{1}$ and $\sigma_{1}$ are the mean and standard deviation of $G_{1}$, and $\mu_{2}, \sigma_{2}$ for $G_{2}$. We define $\Delta=\left|\hat{x}_{1}-\hat{x}_{2}\right|$ as the absolute difference between the observables, where $\hat{x}_{1}$ and $\hat{x}_{2}$ are the measurements of $x_{1}$ and $x_{2}$. We calculate the distribution of the difference, $F(\Delta)$, in the one- $\left(F_{1 \mathrm{D}}(\Delta) \mathrm{d} \Delta\right)$, two$\left(F_{2 \mathrm{D}}(\Delta) \mathrm{d} \Delta\right)$, and three-dimensional $\left(F_{3 \mathrm{D}}(\Delta) \mathrm{d} \Delta\right)$ case. In all cases we took $\mu_{1}=0$ and $\mu_{2}=\mu$ and $\sigma_{1}=\sigma_{2}=\sigma$.
The former simplification is harmless since only the difference $\mu_{1}-\mu_{2}$ is important. The results are:

$$
\begin{aligned}
& F_{1 \mathrm{D}}(\Delta)=\frac{1}{\sqrt{4 \pi \sigma^{2}}}\left\{\exp \left[-\frac{(\Delta+\mu)^{2}}{4 \sigma^{2}}\right]\right. \\
&\left.+\exp \left[-\frac{(\Delta-\mu)^{2}}{4 \sigma^{2}}\right]\right\} \\
& F_{2 \mathrm{D}}(\Delta)=\frac{\Delta}{2 \sigma^{2}} \exp \left[-\frac{1}{2} \frac{\Delta^{2}+\mu^{2}}{2 \sigma^{2}}\right] I_{0}\left(\frac{\Delta \mu}{2 \sigma^{2}}\right),
\end{aligned}
$$

where $I_{0}$ is a modified Bessel function of order zero, and

$$
\begin{aligned}
& F_{3 \mathrm{D}}(\Delta)=\frac{\Delta}{2 \sqrt{\pi} \sigma \mu}\left\{\exp \left[-\frac{1}{2} \frac{(\Delta-\mu)^{2}}{2 \sigma^{2}}\right]\right. \\
&\left.-\exp \left[-\frac{1}{2} \frac{(\Delta+\mu)^{2}}{2 \sigma^{2}}\right]\right\} .
\end{aligned}
$$

Applying L'Hospital's rule we calculate the limit of $F_{3 \mathrm{D}}$ for $\mu \rightarrow 0$ :

$$
\lim _{\mu \rightarrow 0} F_{3 \mathrm{D}}(\Delta) \mathrm{d} \Delta=\frac{\Delta^{2}}{2 \sqrt{\pi} \sigma^{3}} \exp \left[-\frac{\Delta^{2}}{4 \sigma^{2}}\right] .
$$

Figure A1 and Eqs. (A2) and (A3) show that for the twoand three-dimensional cases there is a zero probability of measuring the same value for $x_{1}$ and $x_{2}$, i.e., $\Delta=0$.

\section{Appendix B: The neutron star RX J185635-3754}

After this paper was submitted, Walter (2000) suggested that perhaps the past trajectory of the isolated neutron star RX J185635-3754 intersected that of $\zeta$ Oph. He used HST WFPC2 observations over a three-year baseline to obtain a very accurate proper motion and parallax, and approximated the past orbit by a straight line. He concluded that the neutron star came very near $\zeta$ Oph 1.15 Myr ago in Upper Scorpius for an assumed radial velocity of $-45 \mathrm{~km} \mathrm{~s}^{-1}$.

We have used the simulation machinery described in Sect. 3 to analyse this case in the same way as done there for $\zeta$ Oph and PSR J1932+1059. We have again run three million simulations, covering the range $-50 \pm 50 \mathrm{~km} \mathrm{~s}^{-1}$ for the radial velocity of the neutron star. Figure B1 presents the resulting distribution of minimum separations $D_{\min }$ and associated kinematic ages $\tau_{0}$. This figure can be compared directly with Fig. 3. We have seen in Sect. 3 that 30822 simulations put PSR J1932+1059 within $10 \mathrm{pc}$ of $\zeta \mathrm{Oph}$, and in 4214 of these the encounter took place in Upper Scorpius. By contrast, only 748 simulations put RX J185635-3754 within 10 pc of $\zeta$ Oph, about 1.5 Myr ago. None of these encounters occur within $15 \mathrm{pc}$ of Upper Scorpius. We conclude that it is unlikely that RX J185635-3754 is the remnant of the supernova that gave $\zeta$ Oph its large space velocity. We suspect that this neutron star formed long ago somewhere else in the Galactic plane. 

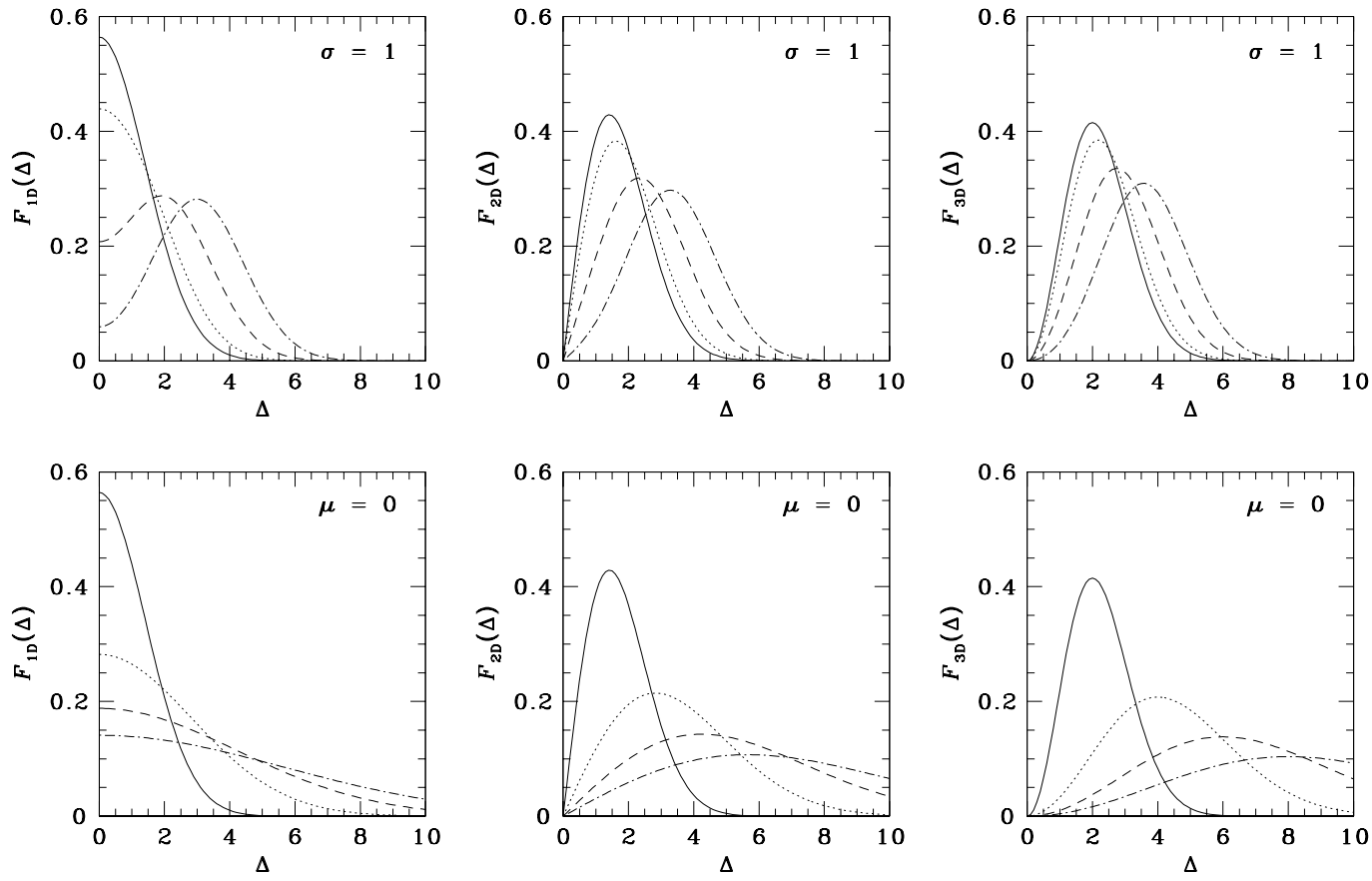

Fig. A1. Left to right: The distribution of absolute differences between two observable quantities, $\Delta$, in one $\left(F_{1 \mathrm{D}}\right)$, two $\left(F_{2 \mathrm{D}}\right)$, and three $\left(F_{3 \mathrm{D}}\right)$ dimensions, taking into account the measurement errors. Top: $\mu=0,1,2$, and 3 are represented by the solid, dotted, dashed, and dot-dashed lines, $\sigma$ is indicated in the top right of each panel. Bottom: $\sigma=1,2,3$, and 4 are represented by the solid, dotted, dashed, and dot-dashed lines, $\mu$ is indicated in the top right of each panel
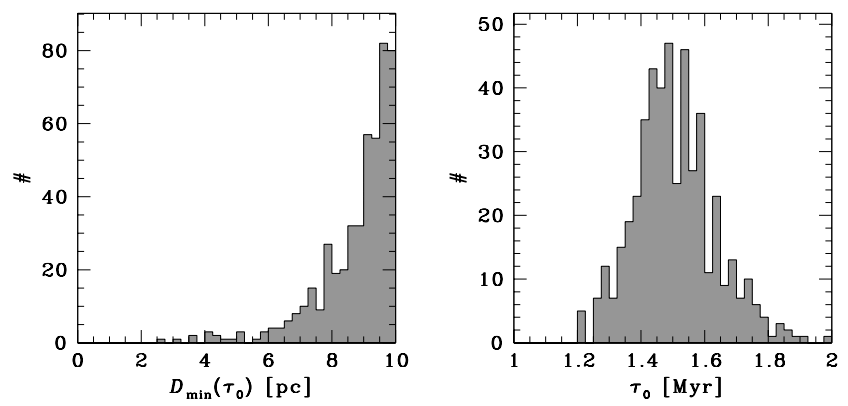

Fig. B1. Left: Distribution of minimum separations, $D_{\min }\left(\tau_{0}\right)$, between $\zeta$ Oph and RX J185635-3754. The solid line denotes the expected distribution of $D_{\min }$, see Sect. 3.3. Right: Distribution of the times $\tau_{0}$ at which the minimum separation was reached

\section{References}

Abt, H. A. 1983, ARA\&A, 21, 343

Abt, H. A., Wang, R., \& Cardona, O. 1991, ApJ, 367, 155

van Albada, T. S. 1961, Bull. Astron. Inst. Netherlands, 15, 301

Barbier-Brossat, M. 1989, Catalogue de Vitesses Radiales Moyennes Stellaires, A\&AS, 80, 67

Barbier-Brossat, M., Petit, M., \& Figon, P. 1994, A\&AS, 108, 603

Beech, M. 1985, Ap\&SS, 117, 69

Benz, W., \& Hills, J. G. 1987, ApJ, 323, 614

Bernacca, P. L., \& Perinotto, M. 1970, Contr. Oss. Astrof. Padova in Asiago, 239, 1

Bignami, G. F., \& Caraveo, P. A. 1996, ARA\&A, 34, 331
Binney, J. J., \& Tremaine, S. 1987, Galactic Dynamics (Princeton University Press)

Blaauw, A. 1944, Bull. Astron. Inst. Netherlands, 10, 29

Blaauw, A. 1952a, Bull. Astron. Inst. Netherlands, 11, 405

Blaauw, A. 1952b, Bull. Astron. Inst. Netherlands, 11, 414

Blaauw, A. 1956, ApJ, 123, 408

Blaauw, A. 1961, Bull. Astron. Inst. Netherlands, 15, 265 (Paper I)

Blaauw, A. 1964, ARA\&A, 2, 213

Blaauw, A. 1978, in Problems of Physics and Evolution of the Universe, ed. L. V. Mirzoyan (Yerevan: Armenian Acad. Sci.), 101

Blaauw, A. 1993, in Massive Stars: Their Lives in the Interstellar Medium, ed. J. P. Cassinelli \& E. B. Churchwell, ASP Conf. Ser., 35, 207

Blaauw, A., \& Morgan, W. W. 1954, ApJ, 119, 625

Blaauw, A., Hiltner, W. A., \& Johnson, H. L. 1959, ApJ, 130, 69 [Erratum: ApJ, 131, 527 (1960)]

Blaauw, A., \& Ramachandran, R. 1998, J. Astroph. Astr., 19, 19

Bohannan, B., \& Garmany, C. D. 1978, ApJ, 223, 908

Bohnenstengel, H.-D., \& Wendker, H. J. 1976, A\&A, 52, 23

Bonnell, I. A. 1999, in The Origin of Stars and Planetary Systems, ed. C. J. Lada \& N. D. Kylafis, NATO ASI Ser. C., 540,479

Bonnell, I. A., Bate, M. R., \& Zinnecker, H. 1998, MNRAS, 298, 93

Brisken, W. F., Benson, J. M., Beasley, A. J., et al. 2000, preprint, [astro-ph/0005324]

Brown, A. G. A., \& Verschueren, W. 1997, A\&A, 319, 811

Brown, A. G. A., de Geus, E. J., \& de Zeeuw, P. T. 1994, A\&A, 289, 101

Van Buren, D., Noriega-Crespo, A., \& Dgani, R. 1995, AJ, 110, 2914 
Burrows, A., Hayes, J., \& Fryxell, B. A. 1995, ApJ, 450, 830 Campbell, R. M. 1995, Ph.D. Thesis (Harvard University)

Campbell, R. M., Bartel, N., Shapiro, I. I., et al. 1996, ApJ, 461, L95

Caraveo, P. A., Bignami, G. F., Mignani, R. P., \& Taff L. G. 1996, ApJ, 461, L91

Caraveo, P. A., Lattanzi, M. G., Massone, G., et al. 1998, A\&A, 329, L1

Carrasco, L., Bisiacchi, G. F., Firmani, C., Costero, R., \& Cruz-González, C. 1980, A\&A, 92, 253

Černis, K. 1993, Baltic Astron., 2, 214

Chevalier, C., \& Ilovaisky, S. A. 1998, A\&A, 330, 201

Comerón, F., Torra, J., \& Gómez, A. E. 1998, A\&A, 330, 975

Cordes, J. M., Romani, R. W., \& Lundgren, S. C. 1993, Nat, 362,133

Crawford, D. L., \& Barnes, J. V. 1970, AJ, 75, 952

Cunha, K., \& Smith, V. V. 1996, A\&A, 309, 892

Dehnen, W., \& Binney, J. J. 1997, MNRAS, 298, 387

Dolan, C. J., \& Mathieu, R. D. 1999, ApJ, 118, 2409

Downs, G. S., \& Reichley, P. E. 1983, ApJS, 53, 169

Duflot, M., Figon, P., \& Meyssonnier, N. 1995, The Wilson Evans Batten Catalogue, A\&AS, 114, 269

Elmegreen, B. G. 1983, MNRAS, 203, 1011

ESA, 1997, The Hipparcos and Tycho Catalogues, ESA SP-1200

Feast, M., \& Whitelock, P. 1997, MNRAS, 291, 683

Frail, D. A., Kulkarni, S. R. 1991, Nat, 352, 785

Frisch, P. C. 1993, Nat, 364, 395

Garmany, C. D., Conti, P. S., \& Massey, P. 1980, ApJ, 242, 1063

Genzel, R., \& Stutzki, J. 1989, ARA\&A, 27, 41

de Geus, E. J. 1992, A\&A, 262, 258

de Geus, E. J., de Zeeuw, P. T., \& Lub, J. 1989, A\&A, 216, 44

Gies, D. R., \& Bolton, C. T. 1986, ApJS, 61, 419

Gies, D. R. 1987, ApJS, 64, 545

Gomez, M., \& Lada, C. J. 1998, AJ, 115, 1524

Häfner, R., \& Drechsel, H. 1986, Ap\&SS, 121, 205

Hansen, B. M. S., \& Phinney, E. S. 1997, MNRAS, 291, 569

Hartman, J. W. 1997, A\&A, 322, 127

Herbst, W. 1975, AJ, 80, 683

Herrero, A., Kudritzki, R. P., Vilchez, J. M., et al. 1992, A\&A, 261,209

van den Heuvel, E. P. J. 1985, in Birth and Evolution of Massive Stars and Stellar Groups, ed. W. Boland, \& H. van Woerden (Dordrecht: Reidel), 107

van den Heuvel, E. P. J., Portegies Zwart, S. F., Bhattacharya, D., \& Kaper, L. 2000, A\&A, in press [astro-ph/0005245]

Hillenbrand, L. A., \& Hartmann, L. W. 1998, ApJ, 492, 540

Hills, J. G. 1983, ApJ, 267, 322

Hoffer, J. B. 1983, AJ, 88, 1420

Høg E., et al. 1998, A\&A, 335, L65

Høg E., Fabricius, C., Makarov, V. V., et al. 2000, A\&A, 357, 367

Hoogerwerf, R., de Bruijne, J. H. J., \& de Zeeuw, P. T. 2000, ApJL, in press [astro-ph/0007436]

Houk, N. 1982, Michigan Spectral Survey, Ann Arbor, Dep. Astron., Univ. Michigan, 3

Humphreys, R. M. 1978, ApJS, 38, 309

Hut, P., \& Bahcall, J. N. 1983, ApJ, 268, 319

Johnson, H. M. 1965, ApJ, 142, 964

de Jong, J. A., Henrichs, H. F., Schijvers, C., et al. 1999, A\&A, 345,172

Jordi, C., Trullols, E., \& Galadí-Enríquez, D. 1996, A\&A, 312, 499
Kaper, L., van Loon, J. Th., Augusteijn, T., et al. 1997, ApJ, 475, L37

Klochkova, V. G., \& Kopylov, I. M. 1985, Bull. Spec. Astrophys. Obs., 20, 3

Kroupa, P. 2000a, New Astron., 4, No. 8, 615

Kroupa, P. 2000b, in Massive Stellar Clusters, ed. A. Lancon, \& C. Boily, ASP Conf. Ser., in press [astro-ph/0001259]

Kroupa, P., Petr, M. G., \& McCaughrean, M. J. 1999, New Astron., 4, No. 7, 495

Kudritzki, R. P., \& Hummer, D. G. 1990, ARA\&A, 28, 303

Kun, M., Balázs, L. G., \& Tóth, I. 1987, Ap\&SS, 134, 211

Kwee, K. K., Muller, C. A., \& Westerhout, G. 1954, Bull. Astron. Inst. Netherlands, 12, 211

Lai, D. 1999, in Stellar Astrophysics Pacific Rim Conf. Proc., (Kluwer Pub.) [astro-ph/9912522]

Leonard, P. J. T. 1989 AJ, 98, 217

Leonard, P. J. T. 1991, AJ, 101, 562

Leonard, P. J. T. 1995, MNRAS, 277, 1080

Leonard, P. J. T., \& Dewey, R. 1993, in Luminous HighLatitude Stars, ed. D. Sasselov, ASP Conf. Ser., 45, 239

Leonard, P. J. T., \& Duncan, M. J. 1988, AJ, 96, 222

Leonard, P. J. T., \& Duncan, M. J. 1990, AJ, 99, 608

Lombardi, J. C. Jr., Rasio, F. A., \& Shapiro, S. L. 1995, ApJ, 445, L117

Lorimer, D. R., Bailes, M., \& Harrison, P. A. 1997, MNRAS 289,592

Lyne, A. G., Anderson, B., \& Salter, M. J. 1982, MNRAS, 201, 503

Maitzen, H. M., Paunzen, E., Pressberger, R., Slettebak, A., \& Wagner, R. M. 1998, A\&A, 339, 782

Mason, B. D., Gies, D. R., Hartkopf, W. I., et al. 1998, AJ, 115,821

McCaughrean, M. J., \& Burkert, A. 2000, The Orion Complex Revisited, ASP Conf. Ser., in press

McCaughrean, M. J., \& Stauffer, J. R. 1994, AJ, 108, 1382

Mikkola, S. 1983a, MNRAS, 203, 1107

Mikkola, S. 1983b, MNRAS, 205, 733

Moffat, A. F. J., et al. 1998, A\&A, 331, 949

Morrell, N., \& Levato, H. 1991, A\&AS, 75, 965

Morse, J. A., Mathieu, R. D., \& Levine, S. E. 1991, AJ, 101, 1495

Packet, W. 1981, A\&A, 102, 17

Palla, F., \& Stahler, S. W. 1999, ApJ, 525, 772

Penny, L. R. 1996, ApJ, 463, 737

Petr, M. G., Coude Du Foresto, V., Beckwith, S. V. W., Richichi, A., \& McCaughrean, M. J. 1998, ApJ, 500, 825

Philp, C. J., Evans, C. R., Leonard, P. J. T., \& Frail, D. A. 1996, AJ, 111, 1220

Portegies Zwart, S. F. 2000, ApJ, in press [astro-ph/0005021]

Poveda, A., Ruiz, J., \& Allen, C. 1967, Bol. de los Obs. Tonantzintla y Tacubaya, 28, 86

Preibisch, T., Balega, Y., Hofmann, K.-H., Weigelt, G., \& Zinnecker, H. 1999, New Astron., 4, 531

Prosser, C. F., Stauffer, J. R., Hartmann, L. W., et al. 1994, ApJ, 421, 517

van Rensbergen, W., Vanbeveren, D., \& de Loore, C. 1996, A\&A, 305, 825

Sahu, M. S. 1992, A Study of the ISM in Puppis-Vela Including the Gum Nebula, Ph.D. Thesis (Groningen Univ.)

Sancisi, R. 1970, A\&A, 4, 387

Sancisi, R., Goss, W. M., Anderson, C., Johansson, L. E. B., \& Winnberg, A. 1974, A\&A, 35, 445

Sargent, A. I. 1979, ApJ, 233, 163 
Sayer, R. W., Nice, D. J., \& Kaspi, V. M. 1996, ApJ, 461, 357

Schaller, G., Schaerer, D., Meynet, G., \& Maeder, A. 1992, A\&AS, 96, 269

Schmidt-Kaler, Th. 1982, in Landolt-Börnstein New Series, Group VI, vol. 2b, ed. K. Schaifers, \& H. H. Voigt (Springer-Verlag), 1

Seyfert, C. K., Hardie, R. H., \& Grenchik, R. T. 1960, ApJ, 132,58

Shull, J. M., \& van Steenberg, M. E. 1985, ApJ, 294, 599

Simon, M., Close, L. M., \& Beck, T. L. 1999, AJ, 117, 1375

Slettebak, A., Wagner, R. M., \& Bertram, R. 1997, PASP, 109, 1

Smith, V. V., Cunha, K., \& Plez, B. 1994, A\&A, 281, L41

Smith, H., \& Eichhorn, H. 1996, MNRAS, 281, 211

Sterken, C. 1988, A\&A, 189, 81

Stickland, D. J., Pike, C. D., Lloyd, C., \& Howarth, I. D. 1987, A\&A, 184, 185

Stone, R. C. 1982, ApJ, 261, 208

Stone, R. C. 1991, AJ, 102, 333

Taylor, J. H., Manchester, R. N., \& Lyne, A. G. 1993, ApJS, 88, 529 (see also http://pulsar.princeton.edu/)

Turon, C., et al. 1992, The Hipparcos Input Catalogue, ESA $\mathrm{SP}-1136$

Uesugi, A., \& Fukada, I. 1970, Contr. Astroph. Kwasan Obs. Univ. Kyoto, 189

Upton, E. K. L. 1971, in The Gum nebula and related problems, ed. S. P. Maran, J. C. Brandt \& T. P. Stecher, NASA SP-332, 119

Vanbeveren, D., De Loore, C., \& Van Rensbergen, W. 1998, A\&AR, 9, 63
Vanbeveren, D., Van Rensbergen, W., \& De Loore, C. 1998, The Brightest Binaries, ASSL, vol. 232

Vitrichenko, E. A., Gershberg, R. E., \& Metik, L. P. 1965, Izv. Krymsk. Ap. Obs., 34, 193

Walker, M. F. 1969, ApJ, 155, 447

Walker, M. F. 1983, ApJ, 271, 642

Walter, F. M. 2000, preprint [astro-ph/0009031]

Warren, W. H., \& Hesser, J. E. 1977a, ApJS, 34, 115

Warren, W. H., \& Hesser, J. E. 1977b, ApJS, 34, 207

Warren, W. H., \& Hesser, J. E. 1978, ApJS, 36, 497

Weigelt, G., Balega, Y., Preibisch, Th., et al. 1999, A\&A, 347, L15

Wilson, R. E. 1953, General Catalogue of Stellar Radial velocities, Carnegie Institution of Washington, Publication 601, Washington, D. C

de Zeeuw, P. T., \& Brand, J. 1985, in Birth and Evolution of Massive Stars and Stellar Groups, ed. W. Boland, \& H. van Woerden (Dordrecht: Reidel), ASP Lib., vol. 120, 95

de Zeeuw, P. T., Hoogerwerf, R., de Bruijne, J. H. J., Brown, A. G. A., \& Blaauw, A. 1999, AJ, 117, 354

de Zeeuw, P. T., Hoogerwerf, R., \& de Bruijne, J. H. J. 2000, in Dynamics of Starclusters and the Milky Way, ed. S. Deiters, et al., ASP Conf. Ser., in press [astro-ph/0007436]

Zinnecker, H., McCaughrean, M. J., \& Wilking, B. A. 1993, in Protostars and Planets III, ed. E. Levy \& J. Lunine (Univ. Arizona Press), 429

Zwicky, F. 1957, Morphological Astronomy (Springer-Verlag, Berlin), 258 